\title{
On the incidence rate of first overtone Blazhko stars in the Large Magellanic Cloud ${ }^{\star}$
}

\begin{abstract}
A. Nagy and G. Kovács
Konkoly Observatory, PO Box 67, 1525 Budapest, Hungary

e-mail: [nagya;kovacs] @konkoly.hu

Received 17 November 2005 / Accepted 29 January 2006

ABSTRACT

Aims. By using the full span of multicolor data on a representative sample of first overtone RR Lyrae stars in the Large Magellanic Cloud (LMC), we revisit the problem of the incidence rate of the amplitude/phase-modulated (Blazhko) stars.

Methods. Multicolor data, obtained by the MAssive Compact Halo Objects (MACHO) project, are utilized through a periodogram averaging method.

Results. The method of analysis enabled us to increase the number of detected multiperiodic variables by $18 \%$, relative to the number obtained by the analysis of the best single color data. We also test the maximum modulation period detectable in the present dataset. We find that variables showing amplitude/phase modulations with periods close to the total time span can still be clearly separated from the class of stars showing period changes. This larger limit on the modulation period, the more efficient data analysis and the longer time span lead to a substantial increase in the incidence rate of the Blazhko stars in comparison to earlier results. We find altogether 99 first overtone Blazhko stars in the full sample of 1332 stars, implying an incidence rate of 7.5\%. Although this rate is nearly twice the one derived earlier, it is still significantly lower than that of the fundamental mode stars in the LMC. The by-products of the analysis (e.g. star-by-star comments, distribution functions of various quantities, etc.) are also presented.
\end{abstract}

Key words. stars: variables: RR Lyrae - stars: fundamental parameters - galaxies: Magellanic Clouds

\section{Introduction}

Current analyses of the databases of the microlensing projects MACHO and Optical Gravitational Lensing Experiment (OGLE) have led to a substantial increase in our knowledge of the frequency of the amplitude/phase-modulated RR Lyrae (Blazhko $[\mathrm{BL}]^{1}$ ) stars. Moskalik \& Poretti (2003) analyzed the OGLE-I data on 215 RR Lyrae stars in the Galactic Bulge. They found incidence rates for the fundamental (RR0) and first overtone (RR1) Blazhko stars of $23 \%$ and $5 \%$, respectively. A similar analysis by Mizerski (2003) on a much larger dataset from the OGLE-II database yielded $20 \%$ and $7 \%$, respectively. These rates, at least for the RR0 stars, are very similar to the ones suggested some time ago by Szeidl (1988) from the various past analyses of rather limited data on Galactic field and globular cluster stars. One gets smaller rates by analyzing the RR Lyrae stars in the Magellanic Clouds. Based on the OGLE-II observations of a sample of 514 stars in the Small Magellanic Cloud (SMC), in a preliminary study, Soszyński et al. (2002) obtained the same rate of $10 \%$ both for the RR0 and RR1 stars. In a similar study, on a very large sample containing 7110 RR Lyrae stars in the LMC, Soszyński et al. (2003) derived $15 \%$ and $6 \%$ for the RR0 and RR1 stars, respectively. In earlier studies on the same galaxy, based on the observations of the MACHO project, Alcock et al. (2000, 2003, hereafter $\mathrm{A} 00$ and $\mathrm{A} 03$, respectively) derived rates of $12 \%$ and $4 \%$

$\star$ Tables 5 and 7 are only available in electronic form at http://www.edpsciences.org

1 See Szeidl \& Kolláth (2000) for a historically more precise possible nomenclature. for the above two classes of variables. One may attempt to relate these incidence rates to the metallicities of the various populations (e.g. Moskalik \& Poretti 2003), but the relation (if it exists) is certainly not a simple one (Kovács 2005; Smolec 2005).

Except for the SMC, all investigations indicate much lower incidence rates for RR1-BL stars than for RR0-BL ones. It is believed that the cause of this difference is internal, i.e. due to real, physical difference, and cannot be fully accounted for by the potentially smaller modulation amplitudes of the RR1 stars. Since this observation may have important consequences on any future modeling of the BL phenomenon, we decided to reanalyze the MACHO database and utilize all available observations (i.e. full time span two-color data). In Sect. 2, we summarize the basic parameters of the datasets and some details of the analysis. Section 3 describes our method for frequency spectrum averaging. The important question of the longest detectable BL period from the present dataset and the concomitant problem of variable classification are dealt with in Sect. 4. Analysis of the RR1 stars with their resulting classifications are presented in Sect. 5. Finally, in Sect. 6, we summarize our main results with a brief discussion of the current state of the field.

\section{Data and method of analysis}

For comparative purposes, in the first part of our analysis, we use the same dataset as the one employed by A00. Our final results are based on the full dataset spanning $~ 7.5$ years. Basic properties of these two sets are listed in Table 1. Both sets contain the same 1354 stars and cover the fields \#2, 3, 5, 6, 9, 10, 11, $12,13,14,15,18,19,47,80,81$, and 82 , essentially sampling 
Table 1. Properties of the LMC RR1 datasets analyzed in this paper.

\begin{tabular}{cccc}
\hline \hline Set & $\left\langle T_{\text {tot }}\right\rangle$ & $\left\langle N_{\mathrm{d}}\right\rangle$ & Colors \\
\hline$\# 1$ & 6.5 & 700 & "r", " $b$ " \\
$\# 2$ & 7.5 & 900 & " $r$ ", " $b$ " \\
\hline
\end{tabular}

Note: $\left\langle T_{\text {tot }}\right\rangle=$ average total time span [yr]; $\left\langle N_{\mathrm{d}}\right\rangle=$ average number of datapoints per variable. Colors: MACHO instrumental magnitudes, see Alcock et al. (1999).

the LMC bar region. Because the selection of the stars was made earlier on simple, preliminary criteria such as period and color, some variables, other than first overtone RR Lyrae stars, were also included. Fortunately, the number of these other variables is only 22, which is small, relative to the full sample.

We employed a standard Discrete Fourier Transformation method by following the implementation of Kurtz (1985). All analyses were performed in the $[0,6] \mathrm{d}^{-1}$ band with 150000 frequency steps, ensuring an ample sampling of the spectrum line profiles, even for the longest time series. The search for the secondary frequencies was conducted through successive prewhitenings in the time domain. The first step consisted of the subtraction of the main pulsation component together with its harmonics up to order three. In all cases, the frequency of the given component was made more accurate by a direct least squares minimization. When the two colors were used simultaneously, we allowed different amplitudes and phases for each and minimized the harmonic mean of the standard deviations of the respective residuals. Although somewhat arbitrarily, we considered the harmonic mean as a useful function for taking into account differences in the data quality.

For the characterization of the signal-to-noise ratio (SNR) of the frequency spectra, we use the following expression

$S N R=\frac{A_{\mathrm{p}}-\left\langle A_{v}\right\rangle}{\sigma_{A_{v}}}$,

where $A_{\mathrm{p}}$ is the amplitude at the highest peak in the spectrum, $\left\langle A_{v}\right\rangle$ is the average of the spectrum, and $\sigma_{A_{v}}$ is its standard deviation, computed by an iterative $4 \sigma$ clipping.

\section{Spectrum Averaging Method (SAM)}

Unlike OGLE, where measurements are taken mostly in the $I_{\mathrm{c}}$ band (e.g. Udalski et al. 1997), the MACHO database has a roughly equal number of measurements in two different colors for the overwhelming majority of the objects (e.g. Alcock et al. 1999). This property of the MACHO database enables us to devise a method that uses both colors to increase the signal detection probability.

It is clear that simple averaging of the time series of the different color bands cannot work because: (i) the two time series are systematically different due to the difference in colors, and (ii) sampling may be different due to the hardware used, the weather, or other reasons. Therefore, we resorted to a spectrum averaging method that uses the frequency spectra of the two time series rather than their directly measured values. Obviously, SAM is also affected by the time series properties mentioned above. However, this effect is a weaker because: (i) we are interested in the positions of the peaks in the spectra and these are the same, regardless of the colors used, and (ii) spectral windows are similar, unless the samplings of the two time series are drastically different. As an example of the fulfilment of this latter condition for the MACHO data, in Fig. 1, we show the spectral windows of the two colors in one representative case.

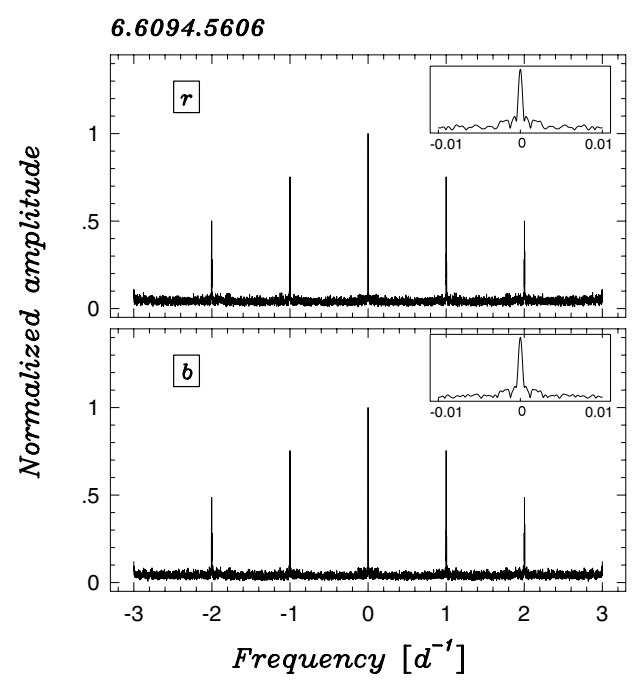

Fig. 1. Example of the similarity of the spectral windows of the MACHO instrumental " $r$ " and " $b$ " time series. Close-ups of the main peaks are displayed in the insets. The MACHO identification number of the star analyzed is shown in the upper left corner of the figure.

To optimize noise suppressing, we compute the summed spectrum by weighting the individual spectra by the inverse of their variances

$S_{i}=\frac{\sigma_{r}^{2} \sigma_{b}^{2}}{\sigma_{r}^{2}+\sigma_{b}^{2}}\left(\frac{1}{\sigma_{r}^{2}} R_{i}+\frac{1}{\sigma_{b}^{2}} B_{i}\right)$,

where $R_{i}$ and $B_{i}$ are the amplitude spectra of the " $r$ " and " $b$ " data and where $\sigma_{r}$ and $\sigma_{b}$ are the standard deviations of the spectra.

Before we discuss the signal detection capability of SAM, it is necessary to give significance levels for periodic signal detection when using the present dataset. Perhaps the simplest way of doing this is to perform a large number of numerical simulations and derive an empirical distribution function of SNR. To achieve this goal, we generated pure Gaussian noise on the observed time base of ten randomly selected stars. For each object, we generated 1000 different realizations and computed the frequency spectra in our standard frequency band of $[0,6] \mathrm{d}^{-1}$. The empirical distribution function was derived from the 10000 SNR values computed on these spectra. Figure 2 shows the resulting functions for the single color and SAM spectra. For further reference, the $1 \%$ significance levels are at 6.9 and 6.5 for the single color and SAM spectra, respectively. All signals that have lower SNR values than these are considered to be not detected in the given time series. The lower cutoff for the SAM spectra is the result of the averaging of independent spectra, which also results in a greater number of independent points in the SAM spectra and concomitantly, in a slightly different shape of the distribution functions (see also Kovács et al. 2002).

We note the following points concerning the signal detection efficiency of SAM. We expect no improvement by applying SAM at very low SNR because the optimally achievable noise suppression is insufficient for the signal to emerge from the noise. In the other extreme, when the signal is strong, the improvement is expected to be minimal, and secure detection is possible without employing SAM. To verify this scenario, we conducted tests with artificial data. The result of one of these tests is shown in Fig. 3. The test data were generated on the 7.5 year time base given by the variable 10.3434 .936 . The signal consisted of a simple sinusoidal with a period of $0.278 \mathrm{~d}$ and amplitude $A$, where $A$ was chosen to be the same in both 


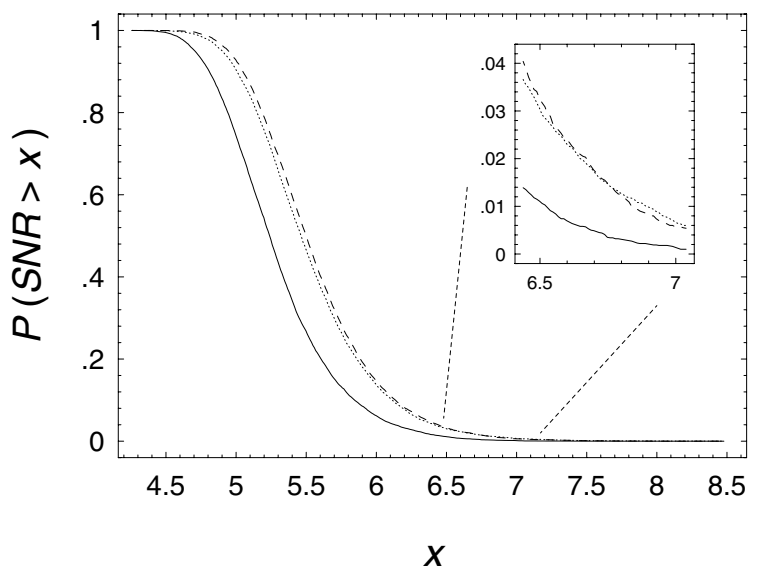

Fig. 2. Probability distribution functions of SNRs of the amplitude spectra of pure Gaussian noise. The time series used in the computation of the spectra were generated on the time base of the " $r$ " data (dotted line), " $b$ " data (dashed line). The result obtained by SAM is shown by solid line.

colors and changed in 100 steps between 0.01 and 0.3 to scan different SNR values. At each value of the amplitude, we added Gaussian noise of $\sigma=0.11$ to the signal. This procedure was repeated for 100 different realizations. For each color and amplitude we computed the average and the standard deviation of the SNR values obtained from these 100 realizations. To characterize the gain in SNR by employing SAM, we also computed the ratio of the SNR values $\left(R_{\mathrm{SNR}}\right)$ as derived from the single color data and by the application of SAM.

We see from Fig. 3 that there is a region where the signal has an amplitude that is too low to be detected in the single color data, but that is not low enough to remain hidden in the SAM spectra. This is the most interesting amplitude/noise regime, the one in which the application of SAM results in new discoveries. At higher amplitudes, SAM "only" leads to an increase in SNR. It is seen that the maximum increase in SNR is at around $\sqrt{2}$, as is to be expected from elementary considerations.

To show non-test examples for the signal detection efficiency of SAM, we exhibit the method at work for the observed data of two variables in Figs. 4 and 5. In both cases, the single color data are insufficient for detection, but the combined spectra clearly show the presence of the signal.

By analyzing all the 1354 variables of Set \#1 we can count the number of cases for which significant components are found after the first prewhitening. The result of this exercise is shown in Table 2. We see that there is strong support of the obvious assumption that the application of two-color data significantly increases the detection probability of faint signals. The gain is $18 \%$, relative to the best single color detection rate.

\section{The phenomenological definition of the $B L$ stars - Separation of the BL and PC variables}

The current loose definition of the BL stars relies purely on the type of the frequency spectra of their light or radial velocity variations. Usually those RR Lyrae stars that exhibit closely spaced peaks in their frequency spectra are called BL variables. Although most of the variables classified as type BL contain only one or two symmetrically spaced peaks at the main pulsation component, complications arise when, after additional prewhitenings, significant components remain, or, when the prewhitening is ambiguous, due to the closeness of the
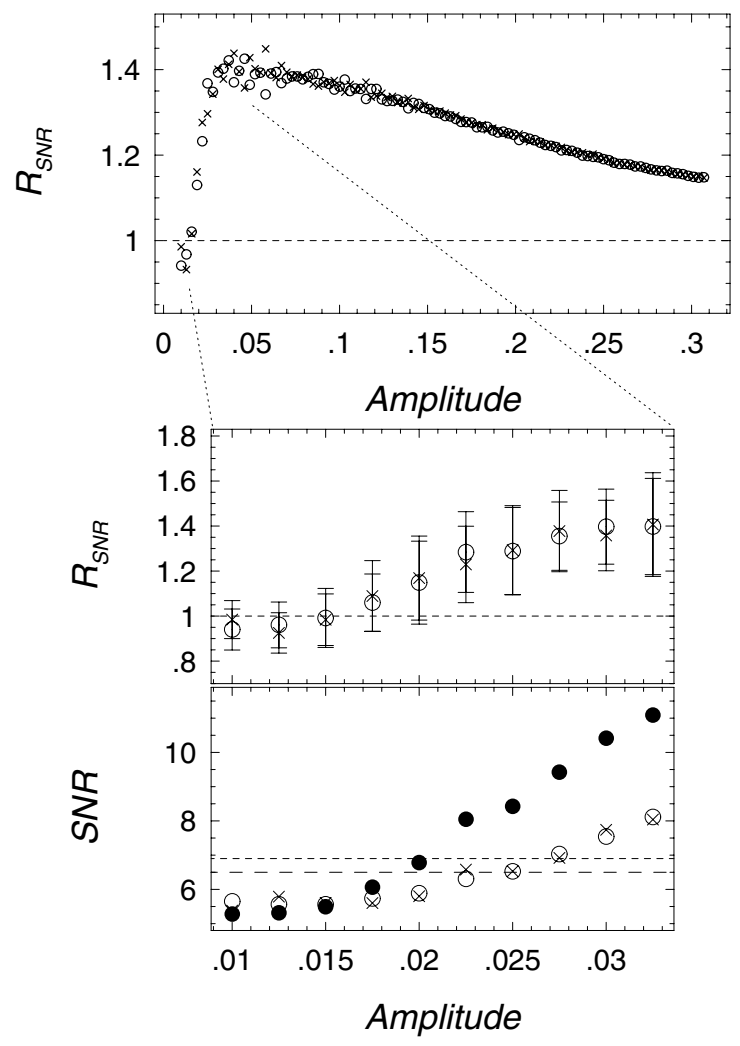

Fig. 3. Top panel: variation of the ensemble average of $R_{\mathrm{SNR}}=$ $S N R_{\mathrm{SAM}} / S N R_{\text {color }}$ as a function of the test amplitude. Open circles refer to the " $r$ " data, crosses to the " $b$ " data. Middle panel: close-up of the low/mid-SNR region of the top diagram. Error bars show the $1 \sigma$ ranges of the values obtained by the various noise realizations. Bottom panel: ensemble average of SNR. The dots are for the SAM values. Short- and long-dashed lines show the $1 \%$ noise probability levels at $S N R=6.9$ and $S N R=6.5$, corresponding to the single-color and SAM spectra, respectively.

secondary components. There are two basic cases of complication: (i) the remnant components are not well-separated and few successive prewhitenings do not lead to a complete elimination of the secondary components, and (ii) there are several well-defined additional peaks, whose patterns are different from the ones usually associated with the BL phenomenon (singlesided for BL1 and symmetrically spaced for BL2 patterns).

Case (i) can be considered as a consequence of some nonstationarity in the data, leading to a nearly continuous spectral representation, whereas case (ii) can be related to some modulation of a more complicated type than that of the BL phenomenon. To make the phenomenological classification of variable types as clear as possible, we adopt the following definitions, which will be supported by the test presented below:

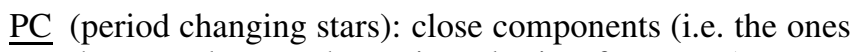
that are close to the main pulsation frequency) cannot be eliminated within three prewhitenening cycles, or, if they can, their separation from the main pulsation component is less than $\sim 1 / T$, where $T$ is the total time span. Furthermore, except for possible harmonics, there are no other significant components in the spectra.

BL (Blazhko stars): there is either one close component, or there are two, spaced symmetrically on both sides of the main pulsation frequency. After the second or third prewhitening, except for possible harmonics, no significant pattern remains or the pattern is of type PC. 


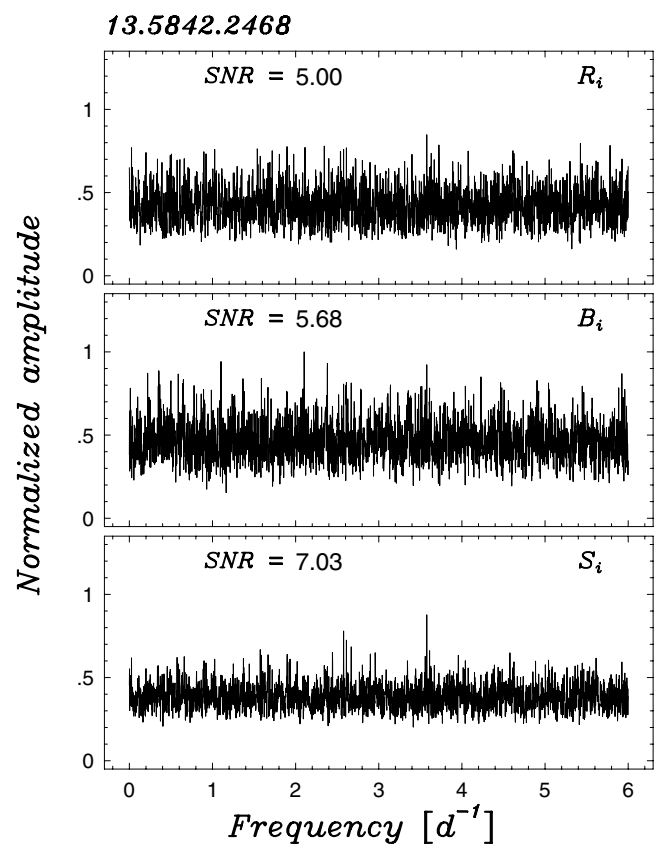

Fig. 4. Example of the signal detection efficiency of SAM at low SNR levels, when the single color data do not show the presence of the signal. The top and middle panels show the frequency spectra for the " $r$ " and " $b$ " data, whereas the bottom one exhibits the SAM result. The MACHO star identification number is shown above the panels, whereas the SNR values are given in the corresponding panels. All spectra are displayed in the same (arbitrary) scale.

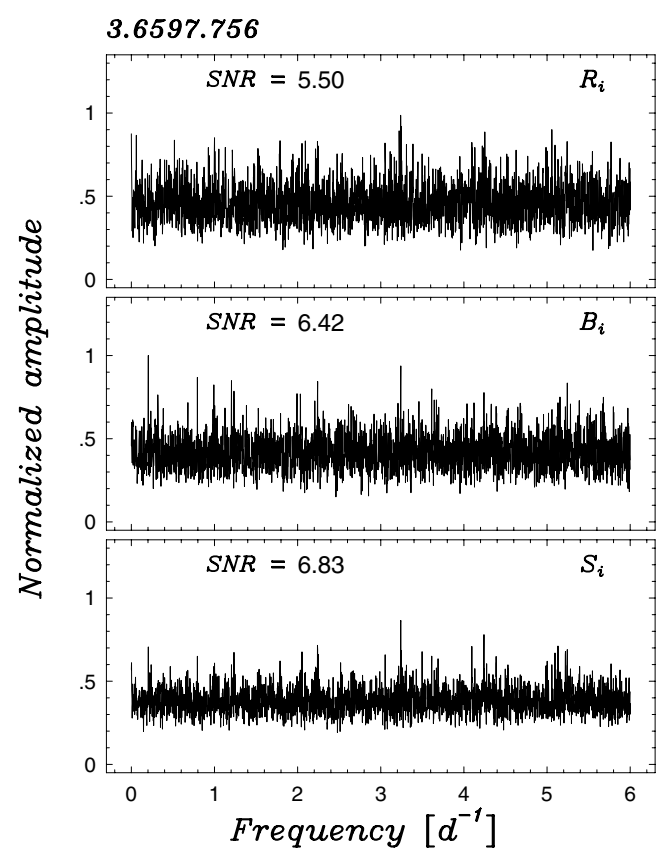

Fig. 5. Example of the signal detection efficiency of SAM for the case when the signal is at the verge of detection in the single color data and SAM increases the reliability of the detection. Notation is the same as in Fig. 4.

$\underline{\mathrm{MC}}$ (stars with closely spaced multiple frequency components): all close components are well-separated (i.e. frequency distances are greater than $\sim 1 / T$ ) and, except if there are only two secondary components on one side of the main pulsation frequency, more than three prewhitenings are necessary to eliminate all of them.
Table 2. Number of single- and multi-periodic variables in Set \#1 of Table 1.

\begin{tabular}{lrrr}
\hline Color & $r$ & $b$ & SAM \\
\hline$N_{1}$ & 978 & 895 & 813 \\
$N_{2}$ & 376 & 459 & 541 \\
$N_{2} /\left(N_{1}+N_{2}\right)$ & $27.8 \%$ & $33.9 \%$ & $40.0 \%$ \\
\hline
\end{tabular}

Note: $N_{1}=$ number of single-periodic variables; $N_{2}=$ number of multiperiodic variables.

Table 3. Definition of the signals used in the BL/PC variability test.

\begin{tabular}{lll}
\hline \hline Type & Time dependence & \\
\hline AM & $A(t)=A \sin (\Omega t+\Phi) ;$ & $\omega(t), \varphi(t)=$ const. \\
PM & $\varphi(t)=A_{\varphi} \sin (\Omega t+\Psi) ;$ & $\omega(t), A(t)=$ const. \\
FM & $\omega(t)=\omega_{0}+A_{\omega} \sin (\Omega t+\Gamma) ; A(t), \varphi(t)=$ const. \\
PC & $\omega(t)=\omega_{0} /\left(1+\beta t / P_{0}\right) ;$ & $A(t), \varphi(t)=$ const. \\
\hline
\end{tabular}

Note: signal form: $x(t)=(1+A(t)) \sin (\omega(t) t+\varphi(t)) ; \omega_{0}=2 \pi / P_{0} ; P_{0}=$ $0.377 ; \Omega=2 \pi / P_{\mathrm{BL}} ; P_{\mathrm{BL}}=\mathrm{BL}$ period; $\mathrm{AM}=$ amplitude modulation; $\mathrm{PM}=$ phase modulation; $\mathrm{FM}=$ frequency modulation; $\mathrm{PC}=$ secular frequency change; and $\Phi, \Psi$, and $\Gamma$ are arbitrary constant phases.

We note that the possible appearance of instrumental effects (i.e. peaks at integer $\mathrm{d}^{-1}$ frequencies) is disregarded in the above classification. Class $\mathrm{MC}$ is equivalent to class $v \mathrm{M}$ used in A00 and A03. Here, the new notation is merely aimed at simplifying the old one. Furthermore, in the previous works, variables with "dominantly" BL2 structures were included in class BL2. Now the above scheme puts them among the MC stars.

Based on the following test, we note that for frequency separations of less than $\sim 1.5 / T$, distinction among the above types becomes more ambiguous because in the simple prewhitening technique followed in this paper, the result depends on the phase of the modulation.

To substantiate the above definitions, we examine in more detail how we can distinguish between the PC and BL phenomena, based solely on the properties of the prewhitened spectra.

We use the time distribution of arbitrarily selected stars to generate artificial time series with the modulated signal parameters given in Table 3. We see that the chosen types of modulation cover nearly all basic cases in the lowest order approximation (i.e. a single main pulsation component with frequency $\omega_{0}$, a linear period change, etc.). No noise is added because we are interested in the differences caused by the various non-stationary components in the residual spectrum, after it was prewhitened by the pulsation component. Nevertheless, noise plays an important role at low modulation levels, but a more detailed study of the complicated problem of non-stationary signal classification in the presence of noise is out of the scope of the present work.

The same pulsation period of $0.377 \mathrm{~d}$ is used for ten randomly selected stars. For each star, we scan the modulation frequency $\Omega$ and the rate of period change $\beta$ at fixed values of all the other parameters. Amplitudes are adjusted to get modulation levels that are $20 \%$ of the peak of the main pulsation component in the frequency spectra. Parameter $\beta$ is changed in the range of $(1-12) \times 10^{-8}$, yielding modulation levels between $20 \%$ and $50 \%$. Each scan is repeated with ten randomly selected phase values. By using the same code for the analysis of these artificial signals as the one employed for the observed data, we get the result shown in Fig. 6. To characterize the efficiency of the prewhitening, we use the ratio $A_{3} / A_{1}$, where $A_{1}$ and $A_{3}$ denote the peak amplitudes after the first and third prewhitenings, respectively. Although in practice we use the SNR of the 
Table 4. Final classification of the 1354 pre-selected variables.

\begin{tabular}{llrr}
\hline \hline Classification & Short description & Number & Inc. rate in RR1 \\
\hline RR1-S & Single-periodic overtone RR Lyrae & 712 & $53.5 \%$ \\
RR1-BL1 & RR1 with one close component & 46 & $3.5 \%$ \\
RR1-BL2 & RR1 with symmetric frequencies & 53 & $4.0 \%$ \\
RR1-MC & RR1 with more close components & 13 & $1.0 \%$ \\
RR1-PC & RR1 with period change & 187 & $14.0 \%$ \\
RR1-D & RR1 with frequencies at integer $d^{-1}$ & 137 & $10.3 \%$ \\
RR1-MI & RR1 with some miscellany & 13 & $1.0 \%$ \\
\hline RR01 & FU/FO double-mode RR Lyrae & 165 & $12.4 \%$ \\
RR01-BL1 & & 1 & $0.1 \%$ \\
RR01-PC & & 5 & $0.4 \%$ \\
\hline RR0-S & Fundamental mode RR Lyrae & 1 & - \\
RR0-BL1 & & 1 & - \\
RR0-BL2 & & 1 & - \\
\hline MDM & Mysterious double-mode & 3 & - \\
BI & Eclipsing binary & 16 & - \\
\hline & & & -
\end{tabular}

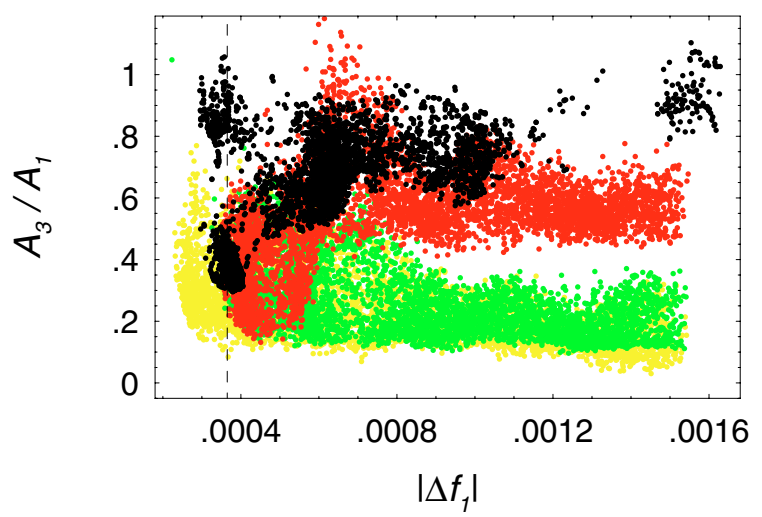

Fig. 6. Testing of the various modulated signals given in Table 3. The ratio of the peak amplitudes after the first and third prewhitenings are plotted as a function of the measured frequency distance (in $\left[\mathrm{d}^{-1}\right]$ ) from the main pulsation component. The dashed vertical line shows the position of the $1 / T$ frequency, where $T$ denotes the total time span. Dots of different shades/colors indicate the various modulation types: yellow/light gray, green/gray, red/dark gray, and black are AM, PM, FM, and PC, respectively. The separation of simple amplitude and phase modulations from the more complicated ones (FM and PC) is clearly visible at $\left|\Delta f_{1}\right|>2 / T$.

frequency spectra to select significant components, here the direct comparison of the amplitudes is more meaningful because for noiseless signals SNR can be high even if the peak amplitude itself is small.

It is clear from the figure that PC signals remain difficult to prewhiten, except perhaps near and below $1 / T$, when any type of signal can be prewhitened due to the proximity of the main pulsation component. Although periodic frequency modulation behaves similarly to secular period change above $1.5 / T$, it can be prewhitened (and therefore confused) with simple amplitude and phase modulations below this limit. When the secular change or periodic modulation of the pulsation frequency is strong, it is more easily separated from simple modulated signals (of types AM and PM, see Table 3). This happens at measured modulation frequencies greater than $\sim 2 / T$ (see the gap starting at $\sim 0.0008 \mathrm{~d}^{-1}$ ). An additional separation between AM and PM signals is observable at modulation frequencies greater than $\sim 5 / T$, where, as expected, the AM type leaves the weakest trace after the third prewhitening.

We conclude that the standard method of prewhitening used for the analysis of the observed data is capable of signaling distinctions between linear period change and signal modulation, but confusion occurs with periodic frequency modulations under observed modulation frequencies of $1.5 / T$. Modulation types AM and FM are also difficult to distinguish, except for short modulation periods, when type AM can be prewhitened more easily. These results support our phenomenological classification of variables given at the beginning of this section, even in the case of long modulation periods.

\section{New detections by using SAM}

Here we readdress the question of the incidence rate of first overtone BL stars by applying the method of analysis and scheme of classification described in the previous sections.

The datasets used in the analysis are described in Sect. 2. The final statistics of classification are shown in Table 4. Additional details of the analysis on a star-by-star basis are given in Table 5. The results presented in Table 4 are based on the analysis of Set \#2, which contains all available two-color data. In comparison to the similar summary of A00 (their Table 7), we see that the most striking difference is the increase of the incidence rate of the BL stars by almost a factor of two. This change can be attributed to the following effects:

- The longer time span: +4 stars - from the comparison of the detections in the " $r$ " data.

- The better quality and higher amplitudes in the " $b$ " data: +28 stars - from the comparison of the Set \#2 " $r$ " and " $b$ " data.

- Extending the allowed range of BL periods up to the length of the total time span: +12 stars - based on the SAM statistics of the Set \#2 results.

- Employing SAM: +9 stars - from the comparison of the Set \#2 " $b$ " and SAM analyses.

- False detections in A00: -7 stars - due to the higher cutoff employed here for the detection limit and because of the somewhat different classification scheme.

For the above reasons, we also find increases in the number of other types of variables. On the other hand, three stars, classified earlier by A00 as RR12 have been re-classified as MDM, or "mysterious double-mode". Basic properties of these stars are summarized in Table 6 . These objects have been re-classified mostly because of their somewhat extreme position in the colormagnitude diagram. Indeed, if we plot the derived average magnitudes and color indices on the color-magnitude diagram of 
Table 6. Properties of the MDM stars.

\begin{tabular}{llccc}
\hline \hline MACHO ID & $P_{1}$ & $P_{2} / P_{1}$ & $V$ & $V-R$ \\
\hline 12.10202 .285 & 0.398 & 0.807 & 18.99 & 0.50 \\
12.10443 .367 & 0.337 & 0.802 & 18.90 & 0.45 \\
9.4278 .179 & 0.327 & 0.805 & 18.69 & 0.29 \\
\hline
\end{tabular}

Alcock et al. (2000a), the three MDM stars fall to the highluminosity red edge of the region populated by RR1 stars. We recall that in the LMC, $\langle V\rangle=19.35$ and $\langle V-R\rangle=0.22$, for the RR1 stars, with $V-R<0.3$ for most of the variables (see Alcock et al. 2004). Although these objects are about 1 mag fainter than the faintest first/second overtone double-mode Cepheids (see Soszyński et al. 2000), it still may not be excluded that they are faint overtone Cepheids rather than bright and very red overtone RR Lyraes. We also note that there is a narrow overlap in the periods of these two classes of stars. One needs more accurate data to understand the status of these intriguing objects.

A slight change in the statistics of the above classification occurs if we consider double identifications due to field overlaps. We consider a star to be double-identified if the following two conditions are satisfied simultaneously: (i) the simple distance derived from the coordinates is smaller than $2 \times 10^{-3}$ degrees, and (ii) the difference between the periods is smaller than $10^{-5} \mathrm{~d}$. We find 52 double-identified objects altogether, including 32 RR1-S, 8 RR01, 5 PC, 1 BL1, and 3 BL2. Except for three marginal cases of PC/S ambiguities, the classifications of the double-identified objects are consistent. The multiple identifications lead to the revised incidence rates of $3.5 \%$ and $3.9 \%$ for the BL1 and BL2 stars, respectively. We see that the changes are insignificant.

\subsection{Properties of the $B L$ and $M C$ stars}

The basic data on the $99 \mathrm{BL}$ stars are summarized in Table 7. As was previously mentioned, in the case of BL2 stars, the modulation frequency $f_{\mathrm{BL}}$ stands for $\left(f_{+}+f_{-}\right) / 2$, where $f_{+}$and $f_{-}$denote the frequencies of the $A_{+}$and $A_{-}$modulation components and $f_{1}$ the main pulsation frequency. This type of averaging is justified, in that among the 53 BL2 variables there are only seven with $\delta f=\left|f_{+}+f_{-}-2 f_{1}\right|>0.0001$, four with $\delta f>0.0002$, and only one (star 15.10068.239) with $\delta f=0.00048$. Although this last value might reflect a statistically significant deviation from an equidistant frequency spacing, the majority of the variables are well within the acceptable deviations expected from observational noise (see A00 for numerical tests).

The BL variables have been selected on the basis of the classification scheme described in Sect. 4. In that scheme, MC-type variables (i.e. those with multiple close periods) have been excluded from the class of BL stars. One may wonder if these variables could also be considered as some subclass of the BL variables. We think that without knowing the underlying physics of the BL phenomenon, it is a question of preference whether or not to consider class MC as a subclass BL. Although we opted against this choice here, we note that there are five variables among the MC stars that show symmetric peaks of BL2-type within the multiple peaks. In Fig. 7, we show two representative cases in which the presence of the BL2 structure is obvious.

Below we give a short description of the frequency spectra of all five stars containing structures of type BL2. In the following explanations we use the notation $v_{0}$ for the main pulsation component.

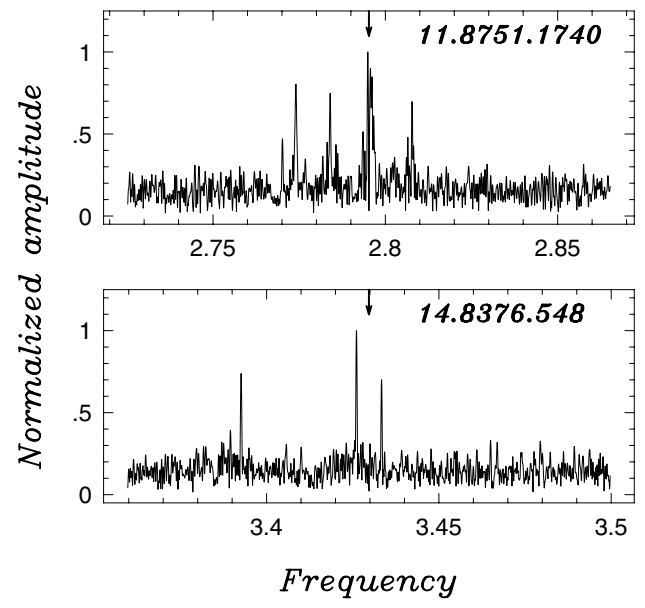

Fig. 7. Examples of type MC variables with BL2 structures. The arrows indicate the position of the (prewhitened) main pulsation component. The spectra are computed by SAM.

11.8751.1740 - the frequency spectrum shows a PC-type remnant at $v_{0}$, a symmetric pair of peaks around it, and an additional component further away (see Fig. 7).

14.8376.548 - the frequency spectrum shows a symmetric pair of closely spaced peaks around $v_{0}$ and an additional peak further away (see Fig. 7).

18.2357.757 - this star is classified as BL2 from the SAM analysis of Set \#1. From Set \#2 we found an additional close component; therefore, the final classification is as MC.

19.4671.684 - the frequency spectrum shows a dominantly BL2 structure, but there are additional peaks spread nearby.

6.6697.1565 - the frequency spectrum shows two superposed BL2 structures, one with large and another one with small frequency separations. The closer pair also has lower amplitudes.

The remaining eight stars from the MC variables show distinct peaks without any apparent BL2-type peak structures. Representative examples of these variables are shown in Fig. 8. We see that these are indeed different from the ones generally classified as type BL.

It is interesting to examine the distribution of the size and the position of the modulation components. For comparison, in Fig. 9, we show the distributions of the modulation amplitudes both for the RR1 and RR0 BL stars. It can be seen that in both classes, there are variables with very high modulation amplitudes, exceeding $0.1 \mathrm{mag}$. This may correspond to $\sim 50 \%$ and $\sim 90 \%$ relative modulation levels for the RR0 and RR1 stars, respectively. In the other extreme, at low modulation amplitudes, we find cases near 0.01-0.02 mag. From the size of the noise and the number of data points, we expect these amplitudes to be the lowest ones detected in this dataset. It is important to note that current investigations by Jurcsik and co-workers (Jurcsik et al. 2005a) suggest that modulation may occur under $0.01 \mathrm{mag}$ among fundamental mode Galactic field Blazhko stars. It is clear that we need more accurate data to check if these low modulation levels are also common in the LMC.

In terms of the relative positions of the modulation components, in Table 8, we show the number of cases obtained in the different datasets with the larger modulation component preceding the main pulsation component. We see that in the case of RR1 stars, there is a nearly $50 \%$ probability of this happenning. This result is essentially independent of BL type, color, and the extent of the dataset. We recall that A03 derived $75 \%$ for this ratio for the RR0 stars in the LMC. 


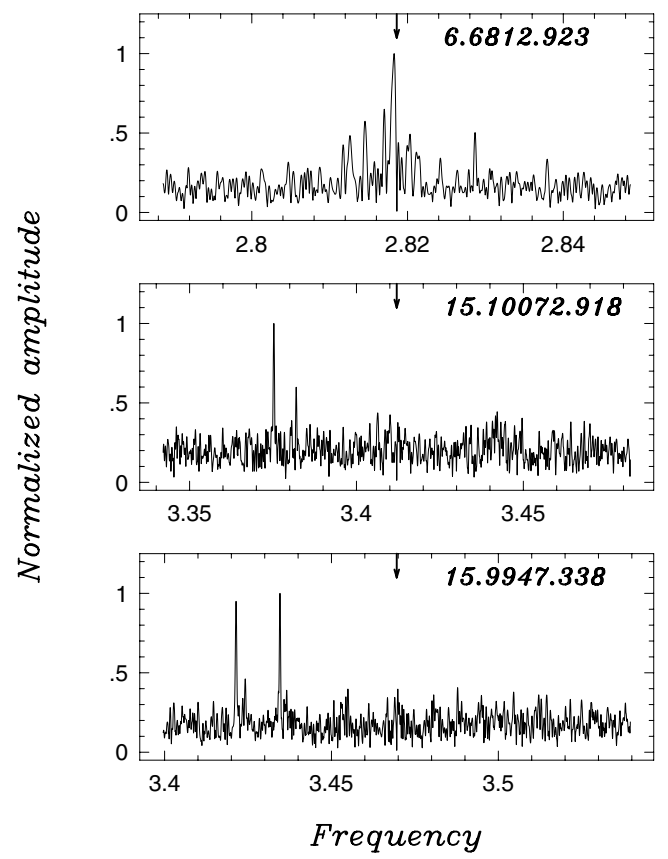

Fig. 8. Examples of type MC variables without symmetric peak structures of type BL2. The arrows indicate the position of the (prewhitened) main pulsation component. Spectra are computed by SAM.

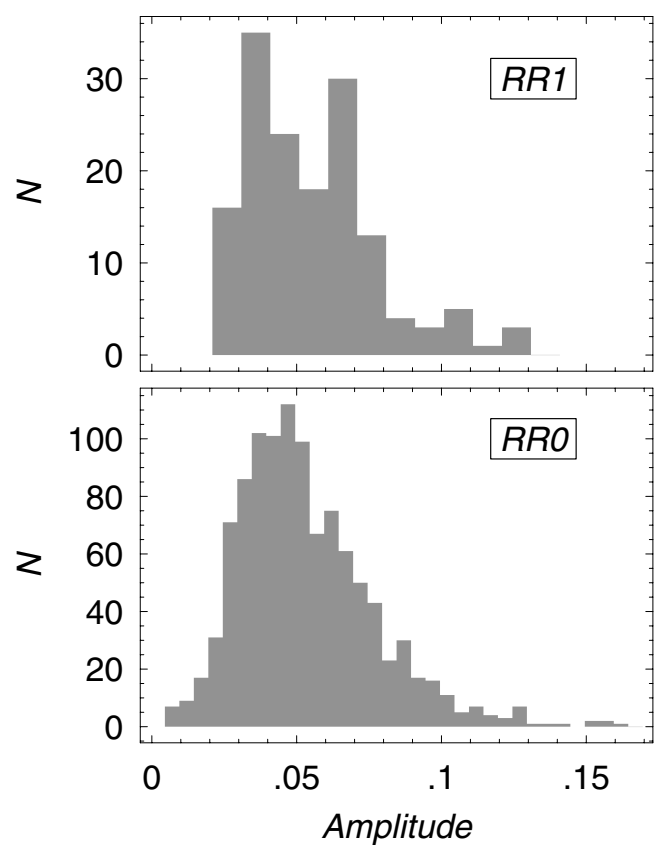

Fig. 9. Upper panel: distribution of the " $b$ " modulation amplitudes of the 99 RR1 stars shown in Table 7. In the case of BL2 stars, the amplitudes of both side peaks are included. Lower panel: as in the upper panel, but for the "V" modulation amplitudes of the 731 RR0 stars of A03.

The present study has largely extended the range of the BL periods known for RR1 stars. The distributions of the modulation frequencies for the RR1 and RR0 stars are shown in Fig. 10. Although the sample is much smaller for the RR1 stars, it is clear that there are more stars among them with short modulation periods than among the RR0 stars. Nevertheless, we should mention that Jurcsik et al. (2005a,b) also found very short modulation periods among RR0 stars, albeit in the Galactic field (i.e. SS Cnc and RR Gem with $P_{\mathrm{BL}}=5.3$ and $7.2 \mathrm{~d}$,
Table 8. Positions of the larger modulation amplitudes.

\begin{tabular}{clccc}
\hline \hline Type & Color & $N_{+}$ & $N_{\text {tot }}$ & $N_{+} / N_{\text {tot }}$ \\
\hline BL1 & " $r$ " & 12 & 32 & 0.38 \\
& "b" & 18 & 44 & 0.41 \\
& "SAM" & 21 & 46 & 0.46 \\
BL2 & "r" & 13 & 22 & 0.59 \\
& "b" & 21 & 46 & 0.46 \\
& "SAM" & 24 & 53 & 0.45 \\
\hline
\end{tabular}

Note: dataset \#2 is used; $N_{+}=$number of cases when the larger modulation amplitude has a frequency greater than that of the main pulsation component; $N_{\text {tot }}=$ total number of BL stars identified in the given color.

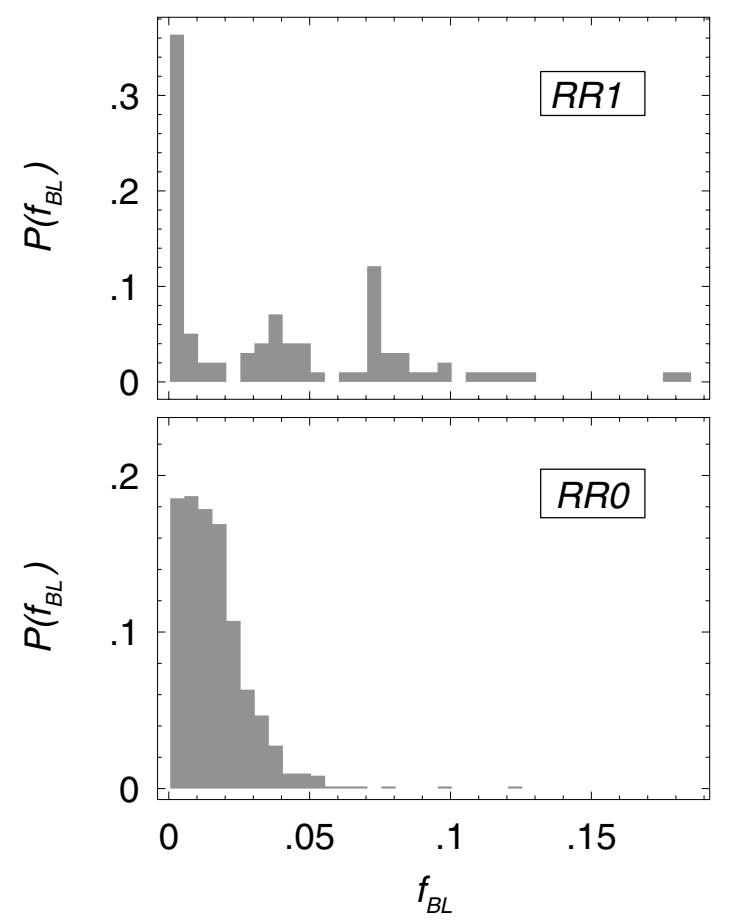

Fig. 10. Upper panel: distribution of the modulation frequencies of the 99 RR1 stars shown in Table 7. Lower panel: as in the upper panel, but for the modulation frequencies of the 731 RR0 stars of A03.

respectively). It is also noted that the considerable surplus in the RR1-BL stars with long modulation periods can be attributed only partially to our lower limit set on $f_{\mathrm{BL}}$. We find that among the 34 stars contributing to the first bin for the plot of RR1 stars in Fig. 10, there are only eleven with $1.0 / T<f_{\mathrm{BL}}<1.5 / T$ and sixteen with $1.0 / T<f_{\mathrm{BL}}<2.0 / T$. This implies a significant peak in the distribution function for $f_{\mathrm{BL}}<0.005$, even with the omission of those objects with very long modulation periods. The lack of such a peak for the RR0 stars might indicate a difference in this respect between the RR0 and RR1 BL stars, but, due to the low sample size for the RR1 stars, we would caution against jumping to this conclusion.

\subsection{Miscellaneous variables}

In Table 9, we list the properties of the variables showing frequency spectra that are difficult to classify. We see that in most cases, the detections are barely above the noise level. Therefore, we cannot exclude that at least some of these variables will turn out to be single-periodic RR1 stars, once more accurate data are available. In the column "Comments" we show the period ratios in cases in which some sort of double-mode pulsation can be 
Table 9. List of miscellaneous stars.

\begin{tabular}{lccccl}
\hline \hline MACHO ID & $v_{0}$ & $v_{M I}$ & $S N R$ & Source & Comments \\
\hline 10.3916 .849 & 3.1389921 & 1.2070180 & 6.61 & SAM & \\
11.8745 .899 & 3.3176002 & 4.7867033 & 6.79 & SAM & $v_{0} / v_{\mathrm{MI}}=0.6931$ \\
13.5719 .713 & 3.0220610 & 3.9155071 & 6.96 & SAM & $v_{0} / v_{\mathrm{MI}}=0.7718$ \\
18.2717 .787 & 2.8643249 & 4.9297051 & 6.55 & SAM & $v_{0} / v_{\mathrm{MI}}=0.5810$ \\
18.3202 .956 & 2.2855398 & 5.1908177 & 6.96 & $\mathrm{r}$ & \\
19.3575 .541 & 3.3698536 & 0.5856270 & 7.91 & $\mathrm{SAM}$ & \\
3.7451 .484 & 3.3048194 & 2.7630866 & 7.85 & $\mathrm{~b}$ & $v_{\mathrm{MI}} / v_{0}=0.8361$ \\
80.6348 .2470 & 2.9571188 & 1.2069597 & 6.65 & $\mathrm{SAM}$ & \\
80.7558 .650 & 2.7828759 & 0.8240633 & 6.99 & $\mathrm{r}$ & \\
81.8519 .1395 & 2.9463890 & 0.8372214 & 6.96 & $\mathrm{r}$ & \\
9.5004 .750 & 3.2877325 & 0.1604569 & 7.42 & SAM & \\
9.5122 .363 & 3.1033541 & 4.1679579 & 6.82 & SAM & $v_{0} / v_{\mathrm{MI}}=0.7446$ \\
9.5241 .382 & 3.1777911 & 1.3826455 & 6.79 & SAM & \\
\hline
\end{tabular}

Note: $v_{0}$ denotes the frequency (in $\left[\mathrm{d}^{-1}\right]$ ) of the main pulsation component; $v_{\mathrm{MI}}$ stands for the frequency of the miscellaneous component with the SNR given in the next column. Period ratios, suggesting some forms of double-mode pulsations, are given in the comment lines.

suspected (without suggesting that those variables are indeed of double-mode ones).

\section{Conclusions}

This work has been motivated by an earlier result of Alcock et al. (2000) on the low incidence rate of the first overtone Blazhko RR Lyrae (RR1-BL) stars in the LMC. They derived a rate of $4 \%$ for these stars, which is a factor of three less than that of the fundamental mode (RR0) stars (see Alcock et al. 2003). Soszyński et al. (2003) obtained a somewhat higher rate of $6 \%$ from the OGLE database. However, Alcock et al. (2000) used MACHO " $r$ " data that have a somewhat lower signal-to-noise ratio for the objects of interest, and, in addition, the data analyzed then spanned a shorter time base than the ones available now. This suggests that the incidence rate could be higher than the one determined earlier. In a full utilization of all available data, we employed a spectrum averaging method that enabled us to increase the detection rate by $18 \%$ in comparison to the best single color (i.e. " $b$ ") rates.

The main conclusion of this paper is that the incidence rate of the RR1-BL stars in the LMC is higher than previously derived from nearly the same database. Even though the rate is now determined to be $7.5 \%$, it is still significantly lower than that of the RR0 stars. This latter rate is $12 \%$; we also expect it to increase slightly when the same method as the one used in this paper on RR1 stars is employed on RR0 stars. This means that at least for the LMC, we can treat the conclusion that RR1-BL stars are significantly less frequent than their counterparts among the RR0 stars as a well-established fact. It is also notable that after filtering out the main pulsation component, the highest amplitude peak appears with equal probability at both sides of the frequency of the main pulsation component. We recall that in the case of RR0 stars, there is a $75 \%$ preference toward the higher frequency side. The smallest modulation amplitudes detected for RR1 stars are near 0.02 mag. For the larger sample of RR0 stars, this limit goes down to $0.01 \mathrm{mag}$. The relative size of the modulation may reach $90 \%$ for RR1 stars, whereas the same limit is only $50 \%$ for the RR0 stars. Furthermore, RR1-BL stars have a relatively large population of short-periodic $\left(P_{\mathrm{BL}}<20 \mathrm{~d}\right)$ variables, while this period regime is nearly empty of RR0 stars in the LMC.
Since the underlying physical mechanism of the BL phenomenon is still unknown, it is also unknown whether these (and other) observational facts will put strong (or even any) constraints on future theories. Nevertheless, since these statistics are based on large samples, their significance is high and surely should not be ignored in theoretical discussions. For example, we note that the very existence of BL1 stars puts the present forms of both currently available models (magnetic oblique rotator/pulsator by Shibahashi 2000; non-radial resonant model by Nowakowski \& Dziembowski 2001; see however Dziembowski \& Mizerski 2004) in jeopardy.

Acknowledgements. This paper utilizes public domain data obtained by the MACHO Project, jointly funded by the US Department of Energy through the University of California, Lawrence Livermore National Laboratory under contract No. W-7405-Eng-48, by the National Science Foundation through the Center for Particle Astrophysics of the University of California under cooperative agreement AST-8809616, and by the Mount Stromlo and Siding Spring Observatory, part of the Australian National University. The support of grant T-038437 of the Hungarian Scientific Research Fund (OTKA) is also acknowledged.

\section{References}

Alcock, C., et al., The MACHO Collab. 1999, PASP, 111, 1539 Alcock, C., et al., The MACHO Collab. 2000, ApJ, 542, 257 (A00) Alcock, C., et al., The MACHO Collab. 2000a, AJ, 119, 2194 Alcock, C., et al., The MACHO Collab. 2003, ApJ, 598, 597 (A03) Alcock, C., et al., The MACHO Collab. 2004, AJ, 127, 334

Dziembowski, W. A., \& Mizerski, T. 2004, Acta Astr., 54, 363 Jurcsik, J., Sódor, Á., Váradi, M., et al. 2005a, A\&A, 430, 1049 Jurcsik, J., Szeidl, B., Nagy, A., \& Sódor, Á. 2005b, Acta Astr., 55, 303 Kovács, G. 2005, A\&A, 438, 227

Kovács, G., Zucker, S., \& Mazeh, T. 2002, A\&A, 391, 369

Kurtz, D. 1985, MNRAS, 213, 773

Mizerski, T. 2003, Acta Astron., 53, 307

Moskalik, P., \& Poretti, E. 2003, A\&A, 398, 213

Nowakowski, R. M., \& Dziembowski, W. A. 2001, Acta Astr., 51, 5 Shibahashi, H. 2000, ASP Conf. Ser., 203, 299

Smolec, R. 2005, Acta Astron., 55, 59

Soszyński, I., Udalski, A., Szymański, M., et al. 2000, Acta Astron., 50, 451 Soszyński, I., Udalski, A., Szymański, M., et al. 2002, Acta Astron., 52, 369 Soszyński, I., Udalski, A., Szymański, M., et al. 2003, Acta Astron., 53, 93 Szeidl, B. 1988, in Multimode Stellar Pulsations, ed. G. Kovács, L. Szabados, \& B. Szeidl (Budapest: Konkoly Observatory), 45

Szeidl, B., \& Kolláth, Z. 2000, ASP Conf. Ser., 203, 281

Udalski, A., Szymański, M., \& Kubiak, M. 1997, Acta Astron., 47, 319 
A. Nagy and G. Kovács: Incidence rate of Blazhko stars, Online Material p 1

\section{Online Material}


A. Nagy and G. Kovács: Incidence rate of Blazhko stars, Online Material p 2

Table 5. Notebook of the analysis.

\begin{tabular}{|c|c|c|c|c|c|}
\hline MACHO ID & $\begin{array}{c}\text { Period } \\
\text { [d] }\end{array}$ & $\begin{array}{l}\text { Type } r \\
\text { (A00) }\end{array}$ & $\begin{array}{l}\text { Type } r, b \\
(\text { SAM) } \\
6.5 \text { years }\end{array}$ & $\begin{array}{l}\text { Type } r, b \\
\text { (SAM) } \\
7.5 \text { years }\end{array}$ & Comments \\
\hline 10.3190 .501 & 0.3527946 & RR1-S & RR1-S & RR1-S & \\
\hline 10.3191 .363 & 0.4060709 & RR01 & RR01 & RR01 & \\
\hline 10.3193 .457 & 0.2977060 & RR1-S & RR1-S & RR1-S & weak peak in $r$ at 0.4477 \\
\hline 10.3193 .514 & 0.2762716 & RR1-S & RR1-S & RR1-S & \\
\hline 10.3310 .723 & 0.3675948 & RR1-S & RR1-S & RR1-S & \\
\hline 10.3311 .612 & 0.3308571 & RR1-S & RR1-S & RR1-S & \\
\hline 10.3314 .787 & 0.3575062 & RR1-S & RR1-S & RR1-S & \\
\hline 10.3314 .873 & 0.3842321 & RR1-S & RR1-S & RR1-S & \\
\hline 10.3314 .916 & 0.3438987 & RR1-S & RR1-S & RR1-S & \\
\hline 10.3315 .758 & 0.2755685 & RR1-S & RR1-S & RR1-S & \\
\hline 10.3432 .666 & 0.3418437 & RR1-S & RR1-PC & RR1-PC & \\
\hline 10.3434 .825 & 0.2776860 & RR1-S & RR1-S & RR1-S & \\
\hline 10.3434 .936 & 0.2769524 & RR1-S & RR1-S & RR1-S & \\
\hline 10.3435 .907 & 0.3295068 & RR1-S & RR1-S & RR1-S & \\
\hline 10.3550 .888 & 0.3317657 & RR1-S & RR1-S & RR1-S & \\
\hline 10.3552 .745 & 0.2922940 & RR1-BL1 & RR1-BL1 & RR1-BL1 & \\
\hline 10.3556 .986 & 0.2961494 & RR1-PC & RR1-PC & RR1-PC & $\Delta f=0.00038$ \\
\hline 10.3557 .1024 & 0.2947668 & RR1-PC & RR1-PC & RR1-BL2 & $\Delta f=0.00043$ \\
\hline 10.3557 .827 & 0.3933231 & RR01 & RR01 & RR01 & \\
\hline 10.3559 .1080 & 0.3501022 & RR1-S & RR1-S & RR1-S & \\
\hline 10.3560 .324 & 0.3387816 & RR1-S & RR1-S & RR1-S & \\
\hline 10.3673 .1018 & 0.3393109 & RR1-S & RR1-S & RR1-S & \\
\hline 10.3680 .988 & 0.4138908 & RR1-S & RR1-S & RR1-S & \\
\hline 10.3797 .865 & 0.3087917 & RR1-PC & RR1-PC & RR1-PC & \\
\hline 10.3797 .923 & 0.3284057 & RR1-S & RR1-S & RR1-S & \\
\hline 10.3798 .634 & 0.3247489 & RR1-S & RR1-S & RR1-S & $2 v_{0}-1$ al. in $r$ \\
\hline 10.3798 .927 & 0.4934297 & RR01 & RR01 & RR01 & $v_{1}>v_{0}$ \\
\hline 10.3799 .828 & 0.2686594 & RR1-S & RR1-S & RR1-S & light curve fuzzy \\
\hline 10.3800 .1073 & 0.2985202 & RR1-S & RR1-S & RR1-S & \\
\hline 10.3802 .446 & 0.3084910 & RR1-S & RR1-PC & RR1-PC & \\
\hline 10.3914 .645 & 0.3452763 & RR1-S & RR1-S & RR1-S & \\
\hline 10.3914 .827 & 0.3554060 & RR1-S & RR1-S & RR1-S & \\
\hline 10.3916 .849 & 0.3185736 & RR1-S & RR1-S & RR1-MI & \\
\hline 10.3916 .911 & 0.2731797 & RR1-S & RR1-S & RR1-S & \\
\hline 10.3920 .693 & 0.3681541 & RR1-S & RR1-S & RR1-D & in $r$ \\
\hline 10.3922 .1213 & 0.3456475 & RR1-S & RR1-S & RR1-S & peak in $r$ at 1.5282 \\
\hline 10.3923 .351 & 0.3339863 & RR1-S & RR1-BL1 & RR1-S & $\mathrm{P}(\mathrm{A} 00) \neq \mathrm{P}(\mathrm{SAM})$ \\
\hline 10.4034 .825 & 0.3470388 & RR1-S & RR1-S & RR1-S & \\
\hline 10.4035 .1095 & 0.3188217 & RR1-S & RR1-BL1 & RR1-BL1 & \\
\hline 10.4035 .1299 & 0.3595035 & RR01 & RR01 & RR01 & \\
\hline 10.4040 .917 & 0.3673826 & RR1-PC & RR1-PC & RR1-PC & \\
\hline 10.4041 .840 & 0.3988199 & RR01 & RR01 & RR01 & \\
\hline 10.4042 .1202 & 0.3703246 & RR01 & RR01 & RR01 & \\
\hline 10.4043 .1392 & 0.4619426 & RR01 & RR01 & RR01 & $v_{1}>v_{0}$ \\
\hline 10.4043 .939 & 0.3518691 & RR1-S & RR1-D & RR1-S & \\
\hline 10.4155 .951 & 0.2907881 & RR1-S & RR1-BL1 & RR1-S & !!!! \\
\hline 10.4160 .1090 & 0.3674925 & RR01 & RR01 & RR01 & \\
\hline 10.4160 .1109 & 0.2781280 & RR1-S & RR1-S & RR1-S & \\
\hline 10.4161 .1053 & 0.2874579 & RR1-BL1 & RR1-BL1 & RR1-BL1 & $\Delta f=.0940$ \\
\hline 10.4165 .348 & 0.3505580 & RR1-PC & RR1-PC & RR1-PC & \\
\hline 10.4275 .3059 & 0.3258438 & RR1-S & RR1-PC & RR1-S & \\
\hline 10.4276 .3455 & 0.3301617 & RR1-S & RR1-S & RR1-S & +strange in $b$, crossID: 9.4276 .488 \\
\hline 10.4281 .2296 & 0.3716398 & RR1-S & RR1-S & RR1-S & \\
\hline 10.4285 .2423 & 0.3480152 & RR1-S & RR1-S & RR1-S & \\
\hline 10.4397 .4675 & 0.3450608 & RR1-S & RR1-S & RR1-S & \\
\hline 10.4398 .4412 & 0.3325706 & RR1-S & RR1-S & RR1-S & crossID: 9.4398 .852 \\
\hline 10.4398 .4446 & 0.3048601 & RR1-S & RR1-S & RR1-S & crossID: 9.4398 .729 \\
\hline 10.4400 .4594 & 0.3322339 & RR1-S & RR1-S & RR1-S & crossID: 5.4400 .1081 \\
\hline 10.4400 .4733 & 0.3254609 & RR1-S & RR1-S & RR1-S & \\
\hline 10.4402 .4727 & 0.3261931 & RR1-S & RR1-S & RR1-S & crossID: 5.4402 .1009 \\
\hline 10.4403 .4871 & 0.4573038 & RR01? & $\mathrm{RR} 01+\mathrm{PC}$ & $\mathrm{RR} 01+\mathrm{PC}$ & $v_{0}$ is changing \\
\hline 10.4520 .4862 & 0.2852017 & RR1-S & RR1-S & RR1-S & crossID: 9.4520 .778 \\
\hline 10.4520 .5022 & 0.3365914 & RR1-S & RR1-S & RR1-S & crossID: 9.4520 .952 \\
\hline 10.4522 .4058 & 0.3589834 & RR01 & RR01 & RR01 & \\
\hline 10.4524 .4611 & 0.3461999 & RR1-S & RR01 & RR01 & crossID: 5.4524 .1212 \\
\hline
\end{tabular}


Table 5. continued.

\begin{tabular}{|c|c|c|c|c|c|}
\hline MACHO ID & $\begin{array}{c}\text { Period } \\
\text { [d] }\end{array}$ & $\begin{array}{l}\text { Type } r \\
\text { (A00) }\end{array}$ & $\begin{array}{l}\text { Type } r, b \\
(\text { SAM) } \\
6.5 \text { years }\end{array}$ & $\begin{array}{l}\text { Type } r, b \\
\text { (SAM) } \\
7.5 \text { years }\end{array}$ & Comments \\
\hline 11.8501 .543 & 0.3703048 & RR1-S & RR1-S & RR1-S & crossID: 14.8501 .3153 \\
\hline 11.8501 .695 & 0.3048447 & RR1-S & RR1-PC & RR1-PC & crossID: 14.8501 .3352 \\
\hline 11.8502 .742 & 0.3581869 & RR1-S & $\mathrm{RR} 01+\mathrm{PC}$ & RR01 & \\
\hline 11.8623 .942 & 0.3084204 & RR1-S & RR1-S & RR1-S & weak RR1-BL1? \\
\hline 11.8625 .1121 & 0.3442731 & RR1-S & RR1-S & RR1-S & \\
\hline 11.8626 .1188 & 0.3496315 & RR1-S & RR1-S & RR1-S & \\
\hline 11.8629 .1482 & 0.3861446 & RR01 & RR01 & RR01 & \\
\hline 11.8744 .658 & 0.4155896 & RR1-PC & RR1-PC & RR1-PC & \\
\hline 11.8744 .752 & 0.2809590 & RR1-S & RR1-S & RR1-S & crossID: 14.8744 .3856 \\
\hline 11.8744 .924 & 0.3114378 & RR1-S & RR1-D & RR1-S & \\
\hline 11.8745 .899 & 0.3014227 & RR1-S & RR1-D & RR1-MI & $v_{0} / v_{1}=.6931$ \\
\hline 11.8750 .1672 & 0.3400457 & RR1-S & RR1-S & RR1-D & \\
\hline 11.8750 .1694 & 0.3577735 & RR01 & RR01 & RR01 & \\
\hline 11.8751 .1740 & 0.3577514 & RR1-MC & $\mathrm{RR} 1-\mathrm{PC}+\mathrm{MC}$ & RR1-PC+MC & symm. pair $+2 \Delta f$ ! \\
\hline 11.8751 .2094 & 0.3160414 & RR1-S & RR1-S & RR1-S & \\
\hline 11.8863 .163 & 0.3312066 & RR1-S & RR1-S & RR1-S & crossID: 14.8863 .1362 \\
\hline 11.8867 .861 & 0.4131937 & RR01 & RR01 & RR01 & \\
\hline 11.8867 .919 & 0.3240251 & RR1-S & RR1-S & RR1-S & \\
\hline 11.8867 .970 & 0.4466407 & RR1-PC & RR1-PC & RR1-PC & \\
\hline 11.8868 .1095 & 0.3185036 & RR1-S & RR1-S & RR1-D & in $r$ \\
\hline 11.8873 .1766 & 0.3277070 & RR1-S & RR1-S & RR1-D & in $b$ \\
\hline 11.8985 .882 & 0.3624996 & RR01 & RR01 & RR01 & \\
\hline 11.8987 .1015 & 0.3341488 & RR1-S & RR1-S & RR1-S & \\
\hline 11.8987 .787 & 0.3041267 & RR1-MC & RR1-MC & RR1-MC & \\
\hline 11.8988 .974 & 0.3247708 & RR1-S & RR1-S & RR1-S & \\
\hline 11.8993 .1532 & 0.3312893 & RR1-S & RR1-S & RR1-BL1? & weak \\
\hline 11.8993 .1649 & 0.3477462 & RR1-S & RR1-S & RR1-D & \\
\hline 11.9107 .989 & 0.2939027 & RR1-S & RR1-S & RR1-S & \\
\hline 11.9109 .1427 & 0.3611056 & RR1-S & RR1-S & RR1-S & \\
\hline 11.9110 .1410 & 0.3405182 & RR1-S & RR1-S & RR1-S & \\
\hline 11.9111 .591 & 0.3145463 & RR1-S & RR1-S & RR1-S & \\
\hline 11.9112 .1035 & 0.3681675 & RR1-S & RR1-S & RR1-S & weak RR1-D \\
\hline 11.9113 .1731 & 0.3664409 & RR1-PC & RR1-PC & RR1-PC & \\
\hline 11.9116 .1382 & 0.3024869 & RR1-PC & RR1-PC & RR1-PC & \\
\hline 11.9228 .828 & 0.3179428 & RR1-S & RR1-D & RR1-D & \\
\hline 11.9230 .894 & 0.2937493 & RR1-S & RR1-S & RR1-S & \\
\hline 11.9231 .545 & 0.3934738 & RR01 & RR01 & RR01 & \\
\hline 11.9233 .1071 & 0.3577559 & RR1-S & RR1-S & RR1-S & \\
\hline 11.9234 .960 & 0.3347677 & RR1-S & RR1-PC & RR1-PC & fuzzy light curve \\
\hline 11.9235 .1034 & 0.4175977 & RR1-PC & RR1-PC & RR1-PC & \\
\hline 11.9349 .558 & 0.4028589 & RR1-PC & RR1-PC & RR1-PC & \\
\hline 11.9350 .903 & 0.3541080 & RR01 & RR01 & RR01 & \\
\hline 11.9352 .1233 & 0.3621517 & RR1-S & RR1-S & RR1-S & peak in $b$ \\
\hline 11.9355 .1235 & 0.3790599 & RR1-PC & RR1-PC & RR1-PC & \\
\hline 11.9355 .1380 & 0.2704291 & RR1-BL2 & RR1-MC & RR1-MC & $\Delta v_{2}=2 \Delta v_{1}$ \\
\hline 11.9357 .1640 & 0.3503957 & RR1-S & RR1-D & RR1-D & \\
\hline 11.9470 .676 & 0.3712763 & RR01 & RR01 & RR01 & \\
\hline 11.9471 .1050 & 0.3542042 & RR1-PC & RR1-PC & RR1-PC & \\
\hline 11.9471 .1172 & 0.3483401 & RR1-S & RR1-S & RR1-S & \\
\hline 11.9471 .1340 & 0.2675705 & RR1-S & RR1-D & RR1-D & \\
\hline 11.9471 .659 & 0.3483822 & RR01 & RR01 & RR01 & \\
\hline 11.9471 .780 & 0.2859825 & RR1-BL1 & RR1-BL1 & RR1-BL1 & \\
\hline 11.9472 .1070 & 0.3355736 & RR1-S & RR1-S & RR1-S & \\
\hline 11.9476 .1456 & 0.3614426 & BI & BI & BI & light curve \\
\hline 11.9478 .1015 & 0.3313227 & RR1-S & RR1-S & RR1-S & \\
\hline 11.9478 .774 & 0.3503890 & RR1-S & RR1-S & RR1-S & \\
\hline 11.9478 .929 & 0.3522860 & RR1-S & RR1-S & RR1-S & weak RR1-D in $b$ \\
\hline 11.9590 .1001 & 0.3600378 & RR1-PC & RR1-PC & RR1-PC & crossID: 14.9590 .3902 \\
\hline 11.9591 .1150 & 0.2523897 & RR1-S & RR1-S & RR1-S & \\
\hline 11.9591 .762 & 0.3392833 & RR1-S & RR1-S & RR1-D & crossID: 14.9591 .3539 \\
\hline 11.9593 .1065 & 0.3276688 & RR1-S & RR1-S & RR1-S & \\
\hline 11.9594 .880 & 0.3222766 & RR1-PC & RR1-PC & RR1-PC & \\
\hline 11.9595 .1162 & 0.4860366 & RR01 & RR01 & RR01 & $v_{1}>v_{0}$ \\
\hline 11.9597 .1164 & 0.3481222 & RR1-S & RR1-S & RR1-S & \\
\hline 11.9711 .943 & 0.2950092 & RR1-S & RR1-S & RR1-S & \\
\hline
\end{tabular}


Table 5. continued.

\begin{tabular}{|c|c|c|c|c|c|}
\hline MACHO ID & $\begin{array}{c}\text { Period } \\
\text { [d] }\end{array}$ & $\begin{array}{l}\text { Type } r \\
\text { (A00) }\end{array}$ & $\begin{array}{c}\text { Type } r, b \\
(\text { SAM) } \\
6.5 \text { years }\end{array}$ & $\begin{array}{l}\text { Type } r, b \\
\text { (SAM) } \\
7.5 \text { years }\end{array}$ & Comments \\
\hline 11.9714 .935 & 0.3029086 & RR1-S & RR1-S & RR1-S & +weak $v_{1} / v_{0}=.7244$ \\
\hline 11.9717 .1185 & 0.3365410 & RR1-S & RR1-S & RR1-S & \\
\hline 11.9717.1404 & 0.2822904 & RR1-S & RR1-S & RR1-S & \\
\hline 11.9719 .1190 & 0.3254644 & RR1-S & RR1-S & RR1-S & \\
\hline 11.9721 .989 & 0.3006416 & RR1-S & RR1-S & RR1-S & \\
\hline 11.9834 .1222 & 0.3103837 & RR1-S & RR1-S & RR1-S & \\
\hline 11.9838 .1201 & 0.3523117 & RR01 & RR01 & RR01 & \\
\hline 11.9838 .834 & 0.3177495 & RR1-S & RR1-S & RR1-S & \\
\hline 11.9841 .828 & 0.3364250 & RR1-S & RR1-S & RR1-S & \\
\hline 12.10078 .864 & 0.3940533 & RR1-S & RR01 & RR01 & \\
\hline 12.10084 .166 & 0.3390483 & RR1-S & RR1-S & RR1-S & \\
\hline 12.10196 .1046 & 0.3411752 & RR1-S & RR1-S & RR1-S & \\
\hline 12.10196 .934 & 0.2856774 & RR1-S & RR1-S & RR1-S & \\
\hline 12.10197.1341 & 0.3166097 & RR1-S & RR1-S & RR1-S & \\
\hline 12.10201 .618 & 0.3801510 & RR1-S & RR1-S & RR1-S & \\
\hline 12.10202 .285 & 0.3981138 & MDM & MDM & MDM & $V: 21.22 \mathrm{mag}, R: 20.8 \mathrm{mag}$ \\
\hline 12.10203 .1158 & 0.2753769 & RR1-S & RR1-S & RR1-S & \\
\hline 12.10203 .1434 & 0.2768082 & RR1-S & RR1-D & RR1-D & \\
\hline 12.10204 .1059 & 0.3573308 & RR1-S & RR1-S & RR1-S & \\
\hline 12.10204 .860 & 0.3638051 & RR1-S & RR1-PC & RR1-S & $\mathrm{P}(\mathrm{A} 00) \neq \mathrm{P}(\mathrm{SAM})$ \\
\hline 12.10319 .816 & 0.3437542 & RR1-S & RR1-D & RR1-D & .3437486 seems better period? \\
\hline 12.10319 .963 & 0.3364132 & RR1-S & RR1-S & RR1-S & \\
\hline 12.10320 .1544 & 0.2805502 & RR1-S & RR1-S & RR1-S & \\
\hline 12.10322 .1142 & 0.3988699 & RR1-S & RR1-S & RR1-S & \\
\hline 12.10324.1144 & 0.3361543 & RR1-S & RR1-S & RR1-S & \\
\hline 12.10324 .852 & 0.3334126 & RR1-S & RR1-S & RR1-S & \\
\hline 12.10325 .1051 & 0.3016384 & RR1-S & RR1-S & RR1-S & \\
\hline 12.10437 .656 & 0.3292892 & RR1-S & RR1-S & RR1-S & \\
\hline 12.10441 .1320 & 0.3466018 & RR1-S & RR1-S & RR1-D & + peak in $r$ only \\
\hline 12.10443 .367 & 0.3365587 & MDM & MDM & MDM & $V: 19.13 \mathrm{mag}, R: 17.99 \mathrm{mag}$ \\
\hline 12.10558 .1379 & 0.3503669 & RR1-S & RR1-S & RR1-S & \\
\hline 12.10560 .1841 & 0.2930150 & RR1-S & RR1-S & RR1-S & \\
\hline 12.10567.1392 & 0.2798609 & RR1-S & RR1-D & RR1-S & \\
\hline 12.10567 .1400 & 0.3366432 & RR1-S & RR1-S & RR1-S & \\
\hline 12.10679.924 & 0.3087344 & RR1-S & RR1-S & RR1-S & \\
\hline 12.10679 .968 & 0.3675864 & RR01 & RR01 & RR01 & \\
\hline 12.10681 .687 & 0.3507141 & RR1-S & RR1-S & RR1-S & \\
\hline 12.10682 .1050 & 0.3583923 & RR1-S & RR1-S & RR1-S & \\
\hline 12.10682 .793 & 0.3636899 & RR01 & RR01 & RR01 & \\
\hline 12.10685 .1084 & 0.3165502 & RR1-S & RR1-S & RR1-S & remnant stuff in $b$ at $v_{0}$ \\
\hline 12.10801 .843 & 0.3650891 & RR1-PC & RR1-PC & RR1-PC & \\
\hline 12.10805 .860 & 0.3411962 & RR1-S & RR1-S & RR1-S & \\
\hline 12.10807 .859 & 0.4968156 & BI & BI & BI & light curve \\
\hline 12.10807.919 & 0.3119925 & RR1-S & RR1-S & RR1-S & \\
\hline 12.10808 .830 & 0.3000152 & RR1-PC & RR1-PC & RR1-PC & \\
\hline 12.10920 .615 & 0.3239811 & RR1-D & RR1-D & RR1-D & \\
\hline 12.10923 .1422 & 0.3780394 & BI & BI & BI & light curve, $v_{1} / v_{0}=1 / 2$ \\
\hline 12.10924.961 & 0.3868628 & RR1-PC & RR1-PC & RR1-PC & \\
\hline 12.10930 .738 & 0.3596019 & RR1-S & RR1-S & RR1-S & \\
\hline 12.11042 .810 & 0.3798227 & RR1-PC & RR1-PC & RR1-PC & \\
\hline 12.11042 .884 & 0.3751375 & RR1-S & RR1-S & RR1-S & \\
\hline 12.11043 .1000 & 0.2643195 & RR1-MI & RR1-D & RR1-D & \\
\hline 12.11044.712 & 0.3516970 & RR1-S & RR1-BL1? & RR1-S & weak remnant; period: .3516991? \\
\hline 12.11048.1190 & 0.2837675 & RR1-S & RR1-S & RR1-S & \\
\hline 12.11172 .776 & 0.3669065 & RR01 & RR01 & RR01 & \\
\hline 12.11283 .284 & 0.3403838 & RR1-D & RR1-D & RR1-D & + remnant in $r$, crossID: 15.11283 .1607 \\
\hline 12.11284.502 & 0.2883280 & RR1-S & RR1-D & RR1-D & \\
\hline 12.9960 .1448 & 0.3086345 & RR1-S & RR1-D & RR1-D & + remnant at $v_{0}$ \\
\hline 12.9961 .937 & 0.3208761 & RR1-S & RR1-S & RR1-S & \\
\hline 12.9962 .1341 & 0.3462807 & RR1-S & RR1-S & RR1-S & \\
\hline 12.9962 .1419 & 0.3687607 & RR01 & RR01 & RR01 & \\
\hline 13.5591 .31 & 0.4054494 & RR1-S & RR1-S & RR1-S & \\
\hline 13.5598 .180 & 0.3030987 & RR1-S & RR1-S & RR1-S & \\
\hline 13.5599 .3267 & 0.2994202 & RR1-S & RR1-S & RR1-PC & \\
\hline 13.5601 .3249 & 0.3614176 & RR1-S & RR1-S & RR1-PC & \\
\hline
\end{tabular}


Table 5. continued.

\begin{tabular}{|c|c|c|c|c|c|}
\hline MACHO ID & $\begin{array}{c}\text { Period } \\
\text { [d] }\end{array}$ & $\begin{array}{l}\text { Type } r \\
\text { (A00) }\end{array}$ & $\begin{array}{l}\text { Type } r, b \\
\text { (SAM) } \\
6.5 \text { years }\end{array}$ & $\begin{array}{l}\text { Type } r, b \\
\text { (SAM) } \\
7.5 \text { years }\end{array}$ & 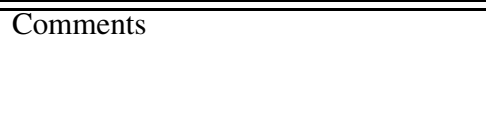 \\
\hline 13.5712 .73 & 0.3391091 & RR1-S & RR1-S & RR1-S & \\
\hline 13.5713 .590 & 0.2836353 & RR1-BL1 & $\mathrm{RR} 1-\mathrm{PC}+\mathrm{MC}$ & RR1-BL1+PC & both $v_{0}$ and $v_{1}$ are changing \\
\hline 13.5714 .442 & 0.3168726 & RR1-BL1 & RR1-BL1 & RR1-BL1 & \\
\hline 13.5714 .490 & 0.3848480 & RR1-S & RR1-S & RR1-S & weak BL1 +weak remnant in $r$ \\
\hline 13.5716 .523 & 0.3419968 & RR1-S & RR1-S & RR1-S & \\
\hline 13.5716 .670 & 0.2934612 & RR1-S & RR1-S & RR1-S & aliases in $b$ \\
\hline 13.5717 .626 & 0.2979910 & RR1-S & RR1-D & RR1-D & \\
\hline 13.5719 .650 & 0.3455553 & RR1-S & RR1-S & RR1-S & \\
\hline 13.5719 .713 & 0.3309000 & RR1-S & RR1-S & RR1-MI & $v_{0} / v_{1}=.7718$ \\
\hline 13.5720 .572 & 0.3720968 & RR1-S & RR1-PC & RR1-PC & \\
\hline 13.5720 .646 & 0.3450355 & RR01 & RR01 & RR01 & \\
\hline 13.5721 .2108 & 0.3355824 & RR1-S & RR1-D & RR1-S & crossID: 6.5721 .289 \\
\hline 13.5721 .2163 & 0.2974574 & RR1-S & RR1-S? & RR1-S & + peak in $b$ only! crossID: 6.5721 .310 \\
\hline 13.5721 .2180 & 0.3221557 & RR1-PC & RR1-PC & RR1-PC & \\
\hline 13.5721 .2290 & 0.3766406 & RR01 & RR01 & RR01 & \\
\hline 13.5722 .3565 & 0.2944695 & RR1-S & RR1-S & RR1-PC & crossID: 6.5722 .682 \\
\hline 13.5722 .4288 & 0.3902119 & RR1-S & RR1-S & BI & $v_{1} / v_{0}=.5000$, light curve. \\
\hline 13.5834 .506 & 0.3538832 & RR1-S & RR1-S & RR1-S & \\
\hline 13.5836 .525 & 0.3604006 & RR01 & RR01 & RR01 & \\
\hline 13.5838 .497 & 0.3697960 & RR01 & RR01 & RR01 & \\
\hline 13.5838 .667 & 0.3425199 & RR1-S & RR1-S & RR1-S & \\
\hline 13.5839 .974 & 0.3232700 & RR1-S & RR1-S & RR1-S & weak RR1-BL1, $\Delta f=-.0010$, aliases \\
\hline 13.5842 .2316 & 0.3604223 & RR01 & RR01 & RR01 & crossID: 6.5842 .396 \\
\hline 13.5842 .2468 & 0.2731424 & RR1-S & RR1-S & RR1-BL1 & \\
\hline 13.5844 .3963 & 0.3702719 & RR1-PC & RR1-PC & RR1-PC & crossID: 6.5844 .872 \\
\hline 13.5955 .540 & 0.2990079 & RR1-S & RR1-S & RR1-S & \\
\hline 13.5957 .647 & 0.2699266 & RR1-S & RR1-S & RR1-S & \\
\hline 13.5958 .518 & 0.3712948 & RR01 & RR01 & $\mathrm{RR} 01+\mathrm{PC}$ & $v_{1}$ is changing \\
\hline 13.5959 .584 & 0.3466458 & RR1-S & RR1-S & RR1-BL1 & $\Delta f=.0010$ \\
\hline 13.5960 .118 & 0.4234486 & RR1-S & RR1-PC & RR1-PC & \\
\hline 13.5960 .884 & 0.3459562 & RR1-S & RR1-S & RR1-S & \\
\hline 13.5963 .2421 & 0.3033331 & RR1-S & RR1-S & RR1-S & \\
\hline 13.5963 .2479 & 0.2764699 & RR1-S & RR1-S & RR1-S & crossID: 6.5963 .363 \\
\hline 13.5964 .3984 & 0.2656551 & RR1-S & RR1-S & RR1-S & \\
\hline 13.5965 .3700 & 0.3669370 & RR1-S & RR1-S & RR1-S & crossID: 6.5965 .890 \\
\hline 13.5965 .3725 & 0.3169630 & RR1-S & RR1-D & RR1-D & crossID: 6.5965 .835 \\
\hline 13.6076 .306 & 0.4270922 & RR1-PC & RR1-PC & RR1-PC & \\
\hline 13.6076 .527 & 0.3524419 & RR1-S & RR1-S & RR1-PC & \\
\hline 13.6077 .638 & 0.2906904 & RR1-PC & RR1-PC & RR1-PC & \\
\hline 13.6079 .604 & 0.3267329 & RR1-S & RR1-S & RR1-S & \\
\hline 13.6080 .541 & 0.3744790 & RR1-PC & RR1-PC & RR1-PC & \\
\hline 13.6080 .594 & 0.4047098 & RR1-PC & RR1-PC & RR1-PC & \\
\hline 13.6080 .628 & 0.4004744 & RR1-PC & RR1-PC & RR1-PC & \\
\hline 13.6081 .990 & 0.3106862 & RR1-S & RR1-S & RR1-S & \\
\hline 13.6083 .535 & 0.3440608 & RR1-S & RR1-PC? & RR1-PC & \\
\hline 13.6084 .2519 & 0.3067479 & RR1-S & RR1-S & RR1-S & crossID: 6.6084 .462 \\
\hline 13.6197.522 & 0.3572369 & RR01 & RR01 & RR01 & \\
\hline 13.6198 .531 & 0.3661721 & RR1-PC & RR1-PC & RR1-PC & \\
\hline 13.6199 .567 & 0.2796456 & RR1-S & RR1-S & RR1-S & \\
\hline 13.6200 .568 & 0.3432525 & RR1-S & RR1-S & RR1-S & \\
\hline 13.6200 .786 & 0.3421512 & RR1-S & RR1-S & RR1-S & \\
\hline 13.6201 .670 & 0.3036885 & RR1-S & RR1-S & RR1-S & $\mathrm{P}(\mathrm{A} 00) \neq \mathrm{P}(\mathrm{SAM})$ \\
\hline 13.6204 .617 & 0.2931520 & RR1-BL1 & RR1-S & RR1-S & \\
\hline 13.6204 .663 & 0.3531123 & RR1-S & RR1-S & RR1-S & \\
\hline 13.6318 .267 & 0.3191239 & RR1-S & RR1-S & RR1-S & \\
\hline 13.6318 .304 & 0.3821733 & RR1-S & RR1-S & RR1-S & \\
\hline 13.6319 .317 & 0.3254238 & RR1-S & RR1-S & RR1-S & \\
\hline 13.6322 .342 & 0.2621495 & RR1-S & RR1-BL1 & RR1-BL1 & \\
\hline 13.6322 .359 & 0.3239249 & RR1-S & RR1-S & RR1-S & \\
\hline 13.6323 .1244 & 0.3109691 & RR1-S & RR1-S & RR1-PC & whitens out \\
\hline 13.6325 .1002 & 0.2908044 & RR1-S & RR1-S & RR1-S & weak RR1-BL1 \\
\hline 13.6326 .1733 & 0.2903530 & RR1-PC & RR1-PC & RR1-PC & \\
\hline 13.6326 .2765 & 0.3304520 & RR1-S & RR1-S & RR1-BL1+PC & crossID: 6.6326 .424 \\
\hline 13.6438 .48 & 0.2936896 & RR1-S & RR1-S & RR1-S & \\
\hline 13.6439 .655 & 0.3304406 & RR1-S & RR1-S & RR1-S & \\
\hline
\end{tabular}


Table 5. continued.

\begin{tabular}{|c|c|c|c|c|c|}
\hline MACHO ID & $\begin{array}{c}\text { Period } \\
\text { [d] }\end{array}$ & $\begin{array}{l}\text { Type } r \\
\text { (A00) }\end{array}$ & $\begin{array}{l}\text { Type } r, b \\
(\text { SAM) } \\
6.5 \text { years }\end{array}$ & $\begin{array}{l}\text { Type } r, b \\
\text { (SAM) } \\
7.5 \text { years }\end{array}$ & 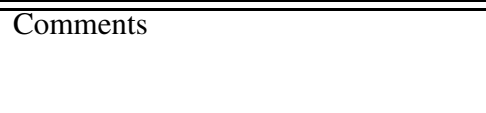 \\
\hline 13.6440 .550 & 0.3185115 & RR1-S & RR1-S & RR1-S & \\
\hline 13.6441 .527 & 0.3435201 & RR1-PC & RR1-PC & RR1-PC & \\
\hline 13.6441 .529 & 0.4025309 & RR1-PC & RR1-PC & RR1-PC & \\
\hline 13.6442 .574 & 0.3224344 & RR1-S & RR1-S & RR1-D & \\
\hline 13.6443 .675 & 0.3792586 & RR1-S & RR1-D & RR1-D & \\
\hline 13.6444 .995 & 0.2804357 & RR1-S & RR1-S & RR1-S & weak $(S / N<6.5) \mathrm{D}$ \\
\hline 13.6445 .617 & 0.3060729 & RR1-S & RR1-PC & RR1-PC & \\
\hline 13.6445 .758 & 0.3482106 & RR1-S & RR1-S & RR1-S & \\
\hline 13.6447 .3202 & 0.3501948 & RR1-S & RR1-S & RR1-S & crossID: 6.6447 .699 \\
\hline 13.6448 .4137 & 0.3431569 & RR1-S & RR1-BL1 & RR1-BL1? & in b only? crossID: 6.6448 .801 \\
\hline 13.6563 .526 & 0.3625070 & RR1-S & RR1-D & RR1-S & \\
\hline 13.6567 .600 & 0.4065653 & RR1-S & RR1-S & RR1-PC & \\
\hline 13.6567 .646 & 0.3482255 & RR1-S & RR1-S & RR1-S & \\
\hline 13.6567 .847 & 0.3007600 & RR1-S & RR1-S & RR1-S & \\
\hline 13.6568.3005 & 0.3380994 & RR1-PC & RR1-PC & RR1-PC & crossID: 6.6568 .484 \\
\hline 13.6569 .4088 & 0.3432541 & RR1-S & RR1-S & RR1-S & crossID: 6.6569 .980 \\
\hline 13.6569 .4284 & 0.3150173 & RR1-S & RR1-S & RR1-S & crossID: 6.6569 .955 \\
\hline 13.6683 .524 & 0.3132242 & RR1-S & RR1-S & RR1-S & \\
\hline 13.6685 .662 & 0.3293156 & RR1-S & RR1-S & RR1-S & \\
\hline 13.6688.699 & 0.3779899 & RR1-S & RR1-MI?+PC? & RR1-PC & \\
\hline 13.6688 .742 & 0.3413834 & RR1-S & RR1-S & RR1-S & \\
\hline 13.6689 .3055 & 0.3050575 & RR1-S & RR1-S & RR1-S & crossID: 6.6689 .563 \\
\hline 13.6691 .4052 & 0.4608689 & RR01 & RR01 & RR01 & $v_{1}>v_{0}$ \\
\hline 13.6802 .544 & 0.3780602 & RR01? & $\mathrm{RR} 01+\mathrm{PC}$ & $\mathrm{RR} 01+\mathrm{PC}$ & both $v_{0}$ and $v_{1}$ are changing \\
\hline 13.6804 .494 & 0.3695517 & RR01 & RR01 & RR01 & \\
\hline 13.6804 .515 & 0.2858770 & RR1-S & RR1-S & RR1-D & \\
\hline 13.6805 .598 & 0.3364643 & RR1-S & RR1-S & RR1-S & +rem.at $v_{0} / .8221$ \\
\hline 13.6806 .585 & 0.3202244 & RR1-S & RR1-S & RR1-S & \\
\hline 13.6806 .664 & 0.3307589 & RR1-D & RR1-D & RR1-D & \\
\hline 13.6807.1020 & 0.3191305 & RR1-S & RR1-S & RR1-S & \\
\hline 13.6807.1093 & 0.2809988 & RR1-S & RR1-S & RR1-S & \\
\hline 13.6807.995 & 0.3256622 & RR1-S & RR1-PC & RR1-PC & symmetric, whitens out, $\Delta f=0.0002$ \\
\hline 13.6808 .1130 & 0.4154337 & BI & BI & BI & light curve \\
\hline 13.6808 .718 & 0.3405710 & RR1-S & RR1-S & RR1-S & \\
\hline 13.6808 .767 & 0.3382012 & RR1-S & RR1-S & RR1-S & strong peak in $b$ only \\
\hline 13.6809 .543 & 0.3574192 & RR1-S & RR1-S & RR1-S & weak RR1-D in $b$ \\
\hline 13.6810 .2845 & 0.3814162 & RR01 & RR01 & RR01 & crossID: 6.6810 .428 \\
\hline 13.6810 .2981 & 0.2883863 & RR1-S & RR1-PC & RR1-BL2 & $\Delta f=.00043$ crossID: 6.6810 .616 \\
\hline 13.6810.2992 & 0.2903975 & RR1-BL2 & RR1-BL2 & RR1-BL2+PC & aliases \\
\hline 13.6811 .4041 & 0.3598933 & RR01 & RR01 & RR01 & crossID: 6.6811 .651 \\
\hline 13.6923 .613 & 0.3430536 & RR1-S & RR1-S & RR1-S & \\
\hline 13.6924 .623 & 0.2951702 & RR1-S & RR1-D & RR1-D & \\
\hline 13.6925 .627 & 0.3566797 & RR01 & RR01 & RR01 & \\
\hline 13.6926 .490 & 0.4383823 & RR1-PC & RR1-PC & RR1-PC & \\
\hline 13.6927 .606 & 0.3631832 & RR01 & RR01 & RR01 & \\
\hline 13.6927 .654 & 0.3558534 & RR1-S & RR1-S & RR1-S & \\
\hline 13.6928.978 & 0.3617956 & RR1-S & RR1-MC+PC? & RR1-PC & PERIOD? $0.36179008 r ; 0.36179099 b$ \\
\hline 13.6929.784 & 0.3549622 & RR1-S & RR1-PC & RR1-PC & \\
\hline 13.6930 .802 & 0.3200681 & RR1-S & RR1-S & RR1-S & \\
\hline 13.6930 .870 & 0.3270085 & RR1-S & RR1-S & RR1-S & \\
\hline 13.6931 .3165 & 0.3590473 & RR1-S & RR1-S & RR1-S & crossID: 6.6931 .704 \\
\hline 13.6931.3219 & 0.3154412 & RR1-S & RR1-S & RR1-D & crossID: 6.6931 .655 \\
\hline 13.6931 .3278 & 0.3228077 & RR1-S & RR1-S & RR1-S & crossID: 6.6931 .649 \\
\hline 13.6933.3973 & 0.3228538 & RR1-S & RR1-PC & RR1-PC & \\
\hline 13.7048 .418 & 0.3490157 & RR01 & RR01 & RR01 & \\
\hline 13.7050 .432 & 0.3347571 & RR1-S & RR1-S & RR1-S & \\
\hline 13.7051 .372 & 0.3373318 & RR1-S & RR1-S & RR1-S & \\
\hline 13.7051 .448 & 0.3657709 & RR1-S & RR1-MC & RR1-PC & \\
\hline 13.7051 .459 & 0.3251078 & RR1-S & RR1-PC & RR1-PC & \\
\hline 13.7052 .2231 & 0.2929019 & RR1-S & RR1-S & RR1-S & \\
\hline 13.7054.3006 & 0.3129400 & RR1-S & RR1-S & RR1-S & crossID: 6.7054 .710 \\
\hline 14.8252 .301 & 0.3424035 & RR1-S & RR1-BL1 & RR1-D & \\
\hline 14.8376 .498 & 0.2901747 & RR1-S & RR1-D & RR1-D & \\
\hline 14.8376 .548 & 0.2915711 & RR1-MC & RR1-MC & RR1-MC & $\Delta f= \pm .0036(37), v_{3}-v_{0}=-.0371$ \\
\hline 14.8380 .776 & 0.3208658 & RR1-S & RR1-S & RR1-S & weak RR1-D in $b$ \\
\hline
\end{tabular}


Table 5. continued.

\begin{tabular}{|c|c|c|c|c|c|}
\hline MACHO ID & $\begin{array}{c}\text { Period } \\
\text { [d] }\end{array}$ & $\begin{array}{l}\text { Type } r \\
\text { (A00) }\end{array}$ & $\begin{array}{l}\text { Type } r, b \\
\text { (SAM) } \\
6.5 \text { years }\end{array}$ & $\begin{array}{l}\text { Type } r, b \\
\text { (SAM) } \\
7.5 \text { years }\end{array}$ & 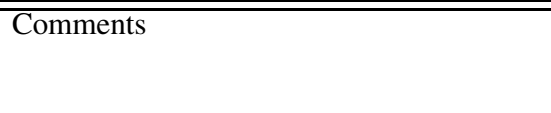 \\
\hline 14.8493 .641 & 0.2804552 & RR1-S & RR1-S & RR1-S & \\
\hline 14.8493 .841 & 0.3377429 & RR1-S & RR1-S & RR1-S & \\
\hline 14.8495 .582 & 0.2971105 & RR1-S & RR1-BL2 & RR1-BL2 & $\Delta f=0.0441$ \\
\hline 14.8497 .534 & 0.2893001 & RR1-PC & RR1-PC & RR1-PC & \\
\hline 14.8498 .847 & 0.3368901 & RR1-S & RR1-S & RR1-S & \\
\hline 14.8499 .544 & 0.3214172 & RR1-S & RR1-S & RR1-S & \\
\hline 14.8500 .1226 & 0.3507788 & RR1-S & RR1-S & RR1-S & $\mathrm{P}(\mathrm{A} 00) \neq \mathrm{P}(\mathrm{SAM})$ \\
\hline 14.8501 .3153 & 0.3703045 & RR1-S & RR1-D & RR1-D & $\mathrm{P}(\mathrm{A} 00) \neq \mathrm{P}(\mathrm{SAM})$ crossID: 11.8501 .543 \\
\hline 14.8501 .3352 & 0.3048425 & RR1-S & RR1-S & RR1-PC & crossID: 11.8501 .695 \\
\hline 14.8613 .787 & 0.3525904 & RR1-S & RR1-MI & RR0-S & $S / N=6$ rem at $v_{0} / .73$ \\
\hline 14.8616 .635 & 0.2676608 & RR1-S & RR1-S & RR1-S & \\
\hline 14.8616 .709 & 0.3538649 & RR1-S & RR1-S & RR1-S & rem at $v_{0} / .7322$ \\
\hline 14.8618 .603 & 0.3399275 & RR1-S & RR1-S & RR1-S & + weak RR1-D \\
\hline 14.8619 .816 & 0.2652884 & RR1-S & RR1-S & RR1-S & \\
\hline 14.8734 .678 & 0.2958678 & RR1-S & RR1-S & RR1-S & \\
\hline 14.8737 .685 & 0.3450778 & RR1-S & RR1-S & RR1-S & +weak RR1-D \\
\hline 14.8737 .823 & 0.3329859 & RR1-S & RR1-S & RR1-S & \\
\hline 14.8739 .801 & 0.2815096 & RR1-S & RR1-D & RR1-D & in $b$ \\
\hline 14.8744 .3716 & 0.4156945 & RR1-PC & RR1-PC & RR1-PC & \\
\hline 14.8744 .3856 & 0.2809577 & RR1-S & RR1-S & RR1-S & crossID: 11.8744 .752 \\
\hline 14.8854.199 & 0.3390661 & RR1-D & RR1-PC+? & RR1-PC & \\
\hline 14.8859 .663 & 0.3103481 & RR1-S & RR1-S & RR1-S & \\
\hline 14.8863 .1362 & 0.3312052 & RR1-S & RR1-S & RR1-S & +weak $v_{0} / v_{1}=.8104$ crossID: 11.8863 .163 \\
\hline 14.8977.1739 & 0.3432359 & RR1-S & RR1-S & RR1-S & \\
\hline 14.8977 .283 & 0.3134319 & RR1-S & RR1-S & RR1-S & \\
\hline 14.8982 .1963 & 0.3676332 & RR01 & RR01 & RR01 & \\
\hline 14.9099 .710 & 0.2820734 & RR1-S & RR1-S & RR1-D & weak \\
\hline 14.9101 .877 & 0.4361277 & RR1-S & BI & BI & $v_{1} / v_{0}=.5000$, lc maybe \\
\hline 14.9220 .799 & 0.3438955 & RR01 & RR01 & RR01 & \\
\hline 14.9223 .737 & 0.3098789 & RR1-BL2 & RR1-BL2 & RR1-BL2 & $\Delta f=0.0316$ \\
\hline 14.9225 .776 & 0.3551288 & RR1-BL1 & RR1-BL1+PC? & RR1-BL1 & +weak $v_{2} / v_{1}=.7477$ \\
\hline 14.9338 .255 & 0.2676678 & RR1-S & RR1-S & RR1-S & \\
\hline 14.9340 .686 & 0.3403248 & RR1-S & RR1-S & RR1-S & +weak peak at 0.92 \\
\hline 14.9342 .919 & 0.3424704 & RR1-S & RR1-S & RR1-D & in $r$ \\
\hline 14.9344 .714 & 0.3473183 & RR1-S & RR1-S & RR1-S & \\
\hline 14.9346 .412 & 0.3434954 & RR1-PC & RR1-PC & RR1-PC & \\
\hline 14.9463 .846 & 0.2749961 & RR1-S & RR1-BL1 & RR1-BL1 & + peak at $\approx v_{0}+1$ \\
\hline 14.9465 .585 & 0.3547704 & RR1-S & RR1-S & RR1-D & \\
\hline 14.9465 .589 & 0.3287855 & RR1-S & RR1-S & RR1-S & \\
\hline 14.9467 .864 & 0.3465627 & RR01 & RR01 & RR01 & \\
\hline 14.9582 .717 & 0.2835774 & RR1-S & RR1-S & RR1-S & \\
\hline 14.9583 .1037 & 0.4060893 & BI & BI & BI & light curve \\
\hline 14.9587 .828 & 0.3512629 & RR01 & RR01 & RR01 & \\
\hline 14.9588 .864 & 0.3716179 & RR1-S & RR1-S & RR1-S & weak RR1-D in $b$ \\
\hline 14.9589 .1469 & 0.3020927 & RR1-S & RR1-S & RR1-S & peak in $b$ at $\approx 1.9$ \\
\hline 14.9590 .3902 & 0.3600392 & RR1-PC & RR1-PC & RR1-PC & crossID: 11.9590 .1001 \\
\hline 14.9591 .3539 & 0.3392787 & RR1-S & RR1-S & RR1-S & crossID: 11.9591 .762 \\
\hline 14.9702 .401 & 0.2754043 & RR1-BL1 & RR1-BL1 & RR1-BL1 & + weak RR1-D \\
\hline 14.9703 .450 & 0.3522143 & RR01 & RR01 & RR01 & \\
\hline 14.9703 .651 & 0.3566657 & RR1-S & RR1-S & RR1-S & \\
\hline 15.10064 .662 & 0.3162848 & RR1-S & RR1-S & RR1-S & \\
\hline 15.10066 .781 & 0.3497410 & RR1-S & RR1-S & RR1-S & \\
\hline 15.10068 .239 & 0.2976949 & RR1-BL2 & RR1-BL2 & RR1-BL2 & $\Delta f=0.0334$ \\
\hline 15.10069 .680 & 0.3745080 & RR1-PC & RR1-S & RR1-PC & \\
\hline 15.10071 .765 & 0.3528949 & RR01 & RR01 & RR01 & \\
\hline 15.10071 .888 & 0.3521986 & RR01 & RR01 & RR01 & \\
\hline 15.10072 .818 & 0.2834267 & RR1-S & RR1-S & RR1-S & RR1-D in $b$ \\
\hline 15.10072 .918 & 0.2930774 & RR1-BL1 & RR1-BL1 & RR1-BL1 & $\Delta f=-.0369$ \\
\hline 15.10194 .3317 & 0.3353776 & RR1-S & RR1-S & RR1-S & \\
\hline 15.10307 .549 & 0.3306027 & RR1-S & RR1-S & RR1-S & \\
\hline 15.10308 .620 & 0.3535518 & RR1-PC & RR1-PC & RR1-PC & \\
\hline 15.10309 .843 & 0.2984189 & RR1-S & RR1-S & RR1-S & \\
\hline 15.10311 .782 & 0.3482903 & RR1-BL2 & RR1-BL2 & RR1-BL2 & $\Delta f=0.0010$ \\
\hline 15.10313 .606 & 0.2925053 & RR1-BL2 & RR1-BL2 & RR1-BL2 & $\Delta f=0.0644$ \\
\hline 15.10427 .514 & 0.3485194 & RR1-S & RR1-D & RR1-D & \\
\hline
\end{tabular}


Table 5. continued.

\begin{tabular}{|c|c|c|c|c|c|}
\hline MACHO ID & $\begin{array}{c}\text { Period } \\
\text { [d] }\end{array}$ & $\begin{array}{l}\text { Type } r \\
\text { (A00) }\end{array}$ & $\begin{array}{c}\text { Type } r, b \\
\text { (SAM) } \\
6.5 \text { years }\end{array}$ & $\begin{array}{l}\text { Type } r, b \\
\text { (SAM) } \\
7.5 \text { years }\end{array}$ & $\overline{\text { Comments }}$ \\
\hline 15.10433 .787 & 0.3394885 & $\overline{\mathrm{RR} 1-\mathrm{PC}}$ & RR1-PC & RR1-PC & \\
\hline 15.10550 .374 & 0.3077982 & RR1-S & RR1-S & RR1-S & \\
\hline 15.10550 .495 & 0.3471121 & RR1-S & RR1-S & RR1-S & \\
\hline 15.10557 .2836 & 0.3541081 & RR1-S & RR01 & RR01 & \\
\hline 15.10557 .2954 & 0.3644224 & RR01 & RR01 & RR01 & \\
\hline 15.10670 .583 & 0.2999786 & RR1-S & RR1-S & RR1-S & + strange rem. \\
\hline 15.10671 .500 & 0.4062024 & RR01 & RR01 & RR01 & \\
\hline 15.10678 .3345 & 0.3344386 & RR1-S & RR1-S & RR1-S & \\
\hline 15.10795 .916 & 0.3074573 & RR1-PC & RR1-PC & RR1-PC & \\
\hline 15.10797 .871 & 0.3485675 & RR01 & RR01 & RR01 & \\
\hline 15.10911 .457 & 0.3408487 & RR1-S & RR1-D & RR1-S & \\
\hline 15.10912 .514 & 0.3723185 & RR1-S & RR1-S & RR1-S & \\
\hline 15.10914 .663 & 0.3670500 & RR1-PC & RR1-PC & RR1-PC & \\
\hline 15.10920 .3094 & 0.3239824 & RR1-S & RR1-D & RR1-D & \\
\hline 15.11036 .255 & 0.2869357 & RR1-BL1 & RR1-BL1 & RR1-BL1 & $\Delta f=+0.0782$ \\
\hline 15.11037 .690 & 0.3611758 & RR1-S & RR1-S & RR1-S & +very weak RR1-PC \\
\hline 15.11040 .667 & 0.3555267 & RR1-S & RR1-S & RR1-S & \\
\hline 15.11040 .828 & 0.3319008 & RR1-S & RR1-S & RR1-S & \\
\hline 15.11154 .325 & 0.3381630 & RR1-S & RR1-S & RR1-S & \\
\hline 15.11155 .469 & 0.2894198 & RR1-S & RR1-S & RR1-S & \\
\hline 15.11156 .431 & 0.3735111 & RR1-S & RR1-S & RR1-S & aliases \\
\hline 15.11157 .243 & 0.3359253 & RR1-S & RR1-S & RR1-S & \\
\hline 15.11276 .323 & 0.3508712 & RR1-S & RR1-S & RR1-S & in $b$ \\
\hline 15.11280 .663 & 0.3299770 & RR1-S & RR1-S & RR1-BL1 & \\
\hline 15.11283 .1607 & 0.3403839 & RR1-S & RR1-S & RR1-S & weak RR1-D in $b$, crossID: 12.11283 .284 \\
\hline 15.9830 .183 & 0.2806755 & RR1-S & RR1-D & RR1-D & \\
\hline 15.9942 .675 & 0.2850893 & RR1-S & RR1-S & RR1-S & \\
\hline 15.9943 .714 & 0.3433162 & RR1-S & RR1-S & RR1-S & \\
\hline 15.9944 .822 & 0.3358499 & RR1-S & RR1-S & RR1-S & \\
\hline 15.9947 .338 & 0.2882211 & RR1-MC & RR1-MC & RR1-MC & $\Delta v_{1}=-.0350, \Delta v_{2}=-.0482$ \\
\hline 15.9948 .593 & 0.3091553 & RR1-S & RR1-S & RR1-S & weak RR1-D in $b$ \\
\hline 15.9950 .876 & 0.2905768 & RR1-S & RR1-S & RR1-S & \\
\hline 15.9952 .3389 & 0.3541299 & RR1-S & RR1-S & RR1-S & \\
\hline 15.9952 .3454 & 0.2615364 & RR1-S & RR1-D & RR1-D & \\
\hline 18.2234.1176 & 0.3537102 & RR01 & RR01 & RR01 & \\
\hline 18.2357.757 & 0.3938249 & RR1-S & RR1-BL2 & RR1-MC & $\Delta f= \pm 0.0247, \Delta v_{2}=0.0539$ \\
\hline 18.2361 .870 & 0.3254790 & RR1-BL2 & RR1-BL2 & RR1-BL2 & $\Delta f=0.0298$ \\
\hline 18.2364 .968 & 0.3563781 & RR1-S & RR1-S & RR1-S & \\
\hline 18.2476 .903 & 0.2623399 & RR1-S & RR1-S & RR1-S & \\
\hline 18.2479 .655 & 0.3187457 & RR1-S & RR1-S & RR1-S & \\
\hline 18.2482 .790 & 0.3991355 & RR1-S & RR1-S & RR1-S & \\
\hline 18.2483 .800 & 0.3342781 & RR1-S & RR1-S & RR1-D & \\
\hline 18.2597 .765 & 0.3579695 & RR1-PC & RR1-PC+MC? & RR1-PC & \\
\hline 18.2597 .933 & 0.2898306 & RR1-S & RR1-S & RR1-S & peak at 1.2 in $b$ \\
\hline 18.2598 .715 & 0.3167194 & RR1-S & RR1-S & RR1-S & \\
\hline 18.2601 .439 & 0.3676446 & RR01 & RR01 & RR01 & \\
\hline 18.2717 .787 & 0.3491224 & RR1-S & RR1-S & RR1-MI & $v_{M I}=v_{0} / .581$ \\
\hline 18.2717.812 & 0.3506581 & RR01 & RR01 & RR01 & \\
\hline 18.2719 .645 & 0.3557849 & RR01 & RR01 & RR01 & \\
\hline 18.2723 .888 & 0.3487799 & RR1-S & RR1-D & RR1-D & \\
\hline 18.2727.794 & 0.3407561 & RR1-S & RR1-S & RR1-S & \\
\hline 18.2839 .754 & 0.3603664 & RR1-S & RR01 & RR01 & \\
\hline 18.2843 .2547 & 0.3523502 & RR01 & RR01 & RR01 & \\
\hline 18.2848 .544 & 0.3538016 & RR1-S & RR1-S & RR1-S & \\
\hline 18.2961 .764 & 0.3368673 & RR1-S & RR1-S & RR1-S & \\
\hline 18.2962 .667 & 0.3642834 & RR1-S & RR1-S & RR1-S & \\
\hline 18.2962 .942 & 0.3283045 & RR1-S & RR1-S & RR1-S & \\
\hline 18.3082 .1147 & 0.3083016 & RR1-S & RR1-S & RR1-S & \\
\hline 18.3084 .852 & 0.3514798 & RR1-S & RR1-S & RR1-S & \\
\hline 18.3085.1997 & 0.2913733 & RR1-S & RR1-S & RR1-S & \\
\hline 18.3086 .1308 & 0.3049418 & RR1-PC & RR1-PC & RR1-PC & \\
\hline 18.3089 .963 & 0.3292058 & RR1-S & RR1-S & RR1-S & \\
\hline 18.3090 .696 & 0.2850000 & RR1-S & RR1-S & RR1-S & \\
\hline 18.3090 .736 & 0.3401601 & RR1-S & RR1-S & RR1-S & peak at 1.2 in $b$ \\
\hline 18.3202 .956 & 0.4375334 & RR1-S & RR1-S & RR1-MI & \\
\hline
\end{tabular}


Table 5. continued.

\begin{tabular}{|c|c|c|c|c|c|}
\hline MACHO ID & $\begin{array}{c}\text { Period } \\
\text { [d] }\end{array}$ & $\begin{array}{l}\text { Type } r \\
\text { (A00) }\end{array}$ & $\begin{array}{l}\text { Type } r, b \\
(\text { SAM) } \\
6.5 \text { years }\end{array}$ & $\begin{array}{l}\text { Type } r, b \\
\text { (SAM) } \\
7.5 \text { years }\end{array}$ & Comments \\
\hline 18.3207 .1039 & 0.2803413 & RR1-S & RR1-S & RR1-S & \\
\hline 18.3211 .679 & 0.3577120 & RR1-S & RR1-S & RR1-S & \\
\hline 18.3326 .1039 & 0.3120644 & RR1-S & RR1-D & RR1-D & \\
\hline 18.3327 .434 & 0.2676823 & RR1-S & RR1-S & RR1-BL1? & $\Delta f=+.1217$, in $r$ \\
\hline 18.3444 .5297 & 0.2919160 & RR1-S & RR1-D & RR1-D & + strange stuff in $r$ \\
\hline 18.3445 .5324 & 0.3193574 & RR1-S & RR1-S & RR1-S & \\
\hline 18.3449 .4292 & 0.3975811 & RR1-S & RR1-S & RR1-S & \\
\hline 19.3575 .541 & 0.2967488 & RR1-S & RR1-S & RR1-MI & $v_{\mathrm{MI}}=0.5856$ \\
\hline 19.3575 .690 & 0.3237353 & RR1-S & RR1-S & RR1-S & \\
\hline 19.3580 .465 & 0.3553299 & RR01 & RR01 & RR01 & \\
\hline 19.3581 .468 & 0.3564439 & RR01 & RR01 & RR01 & \\
\hline 19.3695 .1013 & 0.3184404 & RR1-S & RR1-S & RR1-S & \\
\hline 19.3700 .930 & 0.3597382 & RR1-S & RR1-S & RR1-S & double peak. at $\approx 1.1$ in $b$ \\
\hline 19.3702 .545 & 0.3587570 & RR01 & RR01 & RR01 & \\
\hline 19.3702 .861 & 0.2782078 & RR1-S & RR1-S & RR1-S & \\
\hline 19.3815 .835 & 0.3434257 & RR1-S & RR1-S & RR1-S & \\
\hline 19.3818.902 & 0.3419316 & RR1-S & RR1-S & RR1-S & \\
\hline 19.3819 .806 & 0.3419088 & RR1-S & RR1-S & RR1-S & \\
\hline 19.3821 .759 & 0.3575546 & RR1-S & RR1-S & RR1-S & \\
\hline 19.3821 .794 & 0.3176546 & RR1-S & RR1-PC & RR1-PC & \\
\hline 19.3823 .473 & 0.4089799 & RR1-PC & RR1-PC & RR1-PC & \\
\hline 19.3823 .546 & 0.3379297 & RR1-PC & RR1-PC & RR1-PC & $\mathrm{P}(\mathrm{A} 00) \neq \mathrm{P}(\mathrm{SAM}) .3380507$ \\
\hline 19.3940 .956 & 0.2575013 & RR1-S & RR1-S & RR1-S & \\
\hline 19.3942 .690 & 0.2586072 & RR1-S & RR1-S & RR1-S & + peak in $r$ at $v_{0} \cdot 0.6913$ \\
\hline 19.3942 .803 & 0.3430451 & RR1-S & RR1-S & RR1-S & \\
\hline 19.3943 .856 & 0.3454221 & RR1-S & RR1-PC & RR1-S & \\
\hline 19.3943 .871 & 0.3218319 & RR1-S & RR1-S & RR1-S & \\
\hline 19.4057.1115 & 0.3589699 & RR01 & RR01 & RR01 & no $f_{0}+f_{1}$ and $f_{0}-f_{1}$ \\
\hline 19.4061 .855 & 0.3418551 & RR1-S & RR1-D & RR1-D & in $r$ \\
\hline 19.4062 .1230 & 0.2775809 & RR1-S & RR1-S & RR1-S & $S / N=6.4$ peak in $b$ at 1.4769 \\
\hline 19.4066 .807 & 0.3005532 & RR1-S & RR1-S & RR1-S & \\
\hline 19.4182 .345 & 0.4016251 & RR01 & RR01 & RR01 & \\
\hline 19.4184 .1390 & 0.3569192 & RR1-S & RR1-D & RR1-D & \\
\hline 19.4185 .239 & 0.2773293 & RR1-S & RR1-S & RR1-S & \\
\hline 19.4187.1316 & 0.3457721 & RR1-S & RR1-S & RR1-S & \\
\hline 19.4188.1264 & 0.3702574 & RR1-BL2 & RR1-PC+BL2 & RR1-PC+BL2 & \\
\hline 19.4188.1317 & 0.3347047 & RR1-S & RR1-D & RR1-D & \\
\hline 19.4188 .1333 & 0.2855346 & RR1-S & RR1-S & RR1-S & \\
\hline 19.4188 .195 & 0.2828193 & RR1-BL2 & RR1-BL2 & RR1-BL2 & $\Delta f=0.0121$ \\
\hline 19.4299 .1724 & 0.2915693 & RR1-S & RR1-S & RR1-S & \\
\hline 19.4299 .1932 & 0.2774828 & RR1-S & RR1-S & RR1-S & \\
\hline 19.4303 .852 & 0.2616854 & RR1-S & RR1-S & RR1-S & \\
\hline 19.4308 .880 & 0.3583355 & RR01 & RR01 & RR01 & \\
\hline 19.4420 .1511 & 0.3417203 & RR1-S & RR1-MI? & RR1-S & \\
\hline 19.4427 .769 & 0.3493883 & RR1-S & RR1-S & RR1-S & \\
\hline 19.4429 .678 & 0.3775181 & RR1-PC & RR1-PC & RR1-PC & \\
\hline 19.4429 .703 & 0.3581288 & RR01 & RR01 & RR01 & \\
\hline 19.4541 .1357 & 0.3618792 & RR01 & RR01 & RR01 & \\
\hline 19.4543 .1583 & 0.4111521 & RR1-S & RR1-S & RR1-S & \\
\hline 19.4545 .1503 & 0.3194264 & RR1-S & RR1-S & RR1-S & \\
\hline 19.4547 .725 & 0.3317246 & RR1-S & RR1-S & RR1-S & \\
\hline 19.4662 .4346 & 0.3287573 & RR1-S & RR1-S & RR1-S & \\
\hline 19.4668.2769 & 0.3842229 & RR01 & RR01 & RR01 & crossID: 2.4668 .539 \\
\hline 19.4669 .2905 & 0.3119589 & RR1-PC & RR1-PC & RR1-PC & crossID: 2.4669 .545 \\
\hline 19.4671 .684 & 0.3787444 & RR1-MC & RR1-MC & RR1-MC & $\mathrm{d} v_{1}= \pm .00125$ (and more) \\
\hline 19.4784 .5754 & 0.3517982 & RR1-PC & RR1-PC & RR1-PC & \\
\hline 19.4785 .5170 & 0.4286302 & RR01 & RR01 & RR01 & \\
\hline 19.4785 .5287 & 0.3440254 & RR1-S & RR1-S & RR1-S & crossID: 2.4785 .1203 \\
\hline 19.4786 .4838 & 0.3393137 & RR1-S & RR1-D & RR1-D & \\
\hline 19.4787.4455 & 0.3411946 & RR1-S & RR1-PC & RR1-PC & $v_{1}=v_{0}+1$ (alias) crossID: 2.4787.1072 \\
\hline 19.4789 .4086 & 0.3824050 & RR1-S & RR01 & RR01 & + peak near "-" crossID: 2.4789 .1029 \\
\hline 19.4789 .4120 & 0.3269113 & RR1-S & RR1-S & RR1-S & weak RR1-D crossID: 2.4789 .946 \\
\hline 19.4792 .584 & 0.3290488 & RR1-S & RR1-S & RR1-D & \\
\hline 2.4661 .4689 & 0.3627190 & RR1-S & RR1-S & RR1-S & \\
\hline 2.4661 .4727 & 0.3384451 & RR1-S & RR1-S & RR1-S & \\
\hline
\end{tabular}


Table 5. continued.

\begin{tabular}{|c|c|c|c|c|c|}
\hline MACHO ID & $\begin{array}{c}\text { Period } \\
\text { [d] }\end{array}$ & $\begin{array}{l}\text { Type } r \\
\text { (A00) }\end{array}$ & $\begin{array}{l}\text { Type } r, b \\
(\text { SAM) } \\
6.5 \text { years }\end{array}$ & $\begin{array}{l}\text { Type } r, b \\
\text { (SAM) } \\
7.5 \text { years }\end{array}$ & 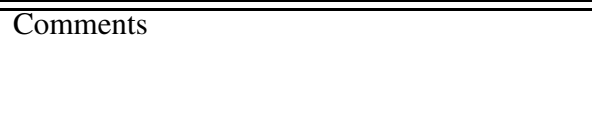 \\
\hline 2.4662 .795 & 0.3461673 & RR1-S & RR1-S & RR1-S & \\
\hline 2.4663 .775 & 0.3902756 & RR1-S & RR1-MI & RR1-PC & aliases \\
\hline 2.4663 .944 & 0.3552641 & RR1-PC & RR1-PC & RR1-PC & whitens out \\
\hline 2.4667 .659 & 0.3243990 & RR1-S & RR1-S & RR1-D & \\
\hline 2.4668 .539 & 0.3842213 & RR01 & RR01 & RR01 & crossID: 19.4668 .2769 \\
\hline 2.4669 .545 & 0.3119586 & RR1-PC & RR1-PC & RR1-PC & crossID: 19.4669 .2905 \\
\hline 2.4785 .1121 & 0.3385838 & RR1-S & RR1-S & RR1-S & \\
\hline 2.4785 .1203 & 0.3440255 & RR1-S & RR1-S & RR1-S & weak RR1-D, crossID: 19.4785 .5287 \\
\hline 2.4787.1072 & 0.3411926 & RR1-S & RR1-S & RR1-S & crossID: 19.4787 .4455 \\
\hline 2.4787 .770 & 0.3357061 & RR1-PC & RR1-PC & RR1-BL2 & $\Delta f=.0005$, symm, $2 v_{0} \pm \Delta f$ \\
\hline 2.4787 .904 & 0.2758016 & RR1-S & RR1-S & RR1-S & \\
\hline 2.4789 .1029 & 0.3824081 & RR01 & RR01 & RR01 & crossID: 19.4789 .4086 \\
\hline 2.4789 .946 & 0.3269118 & RR1-S & RR1-S & RR1-S & weak peak at 1.35 in $b$, crossID: 19.4789 .4120 \\
\hline 2.4790 .736 & 0.2719880 & RR1-S & RR1-S & RR1-D & \\
\hline 2.4791 .631 & 0.8358569 & BI & BI & BI & light curve \\
\hline 2.4904 .1425 & 0.3455885 & RR1-S & RR01 & RR01 & no $f_{0}+f_{1}$ \\
\hline 2.4904 .1651 & 0.3503548 & RR01 & RR01 & RR01 & no $f_{0}-f_{1}$ \\
\hline 2.4906 .826 & 0.3857453 & RR1-S & RR01 & RR01 & no $f_{0} \pm f_{1}$, changing? \\
\hline 2.4908 .1321 & 0.3440402 & RR01 & RR01 & RR01 & \\
\hline 2.4908 .826 & 0.3765790 & RR1-PC & RR1-PC & RR1-PC & \\
\hline 2.5023 .5787 & 0.3245356 & RR1-MC & RR1-MC & RR1-MC & $\Delta v_{1}=.0129, \Delta v_{2}=.0080$ \\
\hline 2.5023 .6558 & 0.2674391 & RR1-S & RR1-S & RR1-S & \\
\hline 2.5024 .3210 & 0.3675679 & RR1-S & RR1-S & RR01 & \\
\hline 2.5028 .411 & 0.2809399 & RR1-S & RR1-S & RR1-S & \\
\hline 2.5031 .701 & 0.2763638 & RR1-S & RR1-S & RR1-S & \\
\hline 2.5031 .766 & 0.2935514 & RR1-S & RR1-S & RR1-S & \\
\hline 2.5032 .703 & 0.3077973 & RR1-S & RR1-PC? & RR1-BL1 & \\
\hline 2.5033 .535 & 0.2627292 & RR1-S & RR1-D & RR1-D & \\
\hline 2.5145 .3578 & 0.3375009 & RR1-S & RR1-S & RR1-S & \\
\hline 2.5147 .1527 & 0.3353089 & RR1-S & RR1-S & RR1-S & \\
\hline 2.5148 .1207 & 0.2946848 & RR1-BL2 & RR1-BL2 & RR1-BL2 & $\Delta f= \pm 0.0353$ \\
\hline 2.5148 .713 & 0.3212517 & RR1-PC & RR1-PC & RR1-BL2 & $\Delta f=.0007$, whitens out \\
\hline 2.5149 .515 & 0.2804797 & RR1-S & RR1-S & RR1-S & \\
\hline 2.5149 .551 & 0.2993013 & RR1-S & RR1-BL1 & RR1-S & \\
\hline 2.5150 .896 & 0.3371211 & RR1-S & RR1-S & RR1-S & \\
\hline 2.5151 .924 & 0.3146242 & RR1-PC & RR1-PC & RR1-PC & \\
\hline 2.5151 .939 & 0.2959491 & RR1-S & RR1-S & RR1-S & \\
\hline 2.5151 .955 & 0.3837021 & RR1-S & RR1-S & RR1-S & + very weak RR1-PC-like rem. \\
\hline 2.5151 .982 & 0.3445221 & RR1-S & RR1-S & RR1-S & \\
\hline 2.5152 .635 & 0.3347839 & RR1-S & RR1-S & RR1-S & \\
\hline 2.5152 .763 & 0.3246989 & RR1-S & RR1-S & RR1-D & $v_{2}=1.9773$ strong in $b$ \\
\hline 2.5266 .3857 & 0.3408903 & RR1-S & RR1-S & RR1-S & \\
\hline 2.5266 .3864 & 0.2793840 & RR1-BL1 & RR1-BL1 & RR1-BL1 & +RR1-PC RR1-BL1 changes \\
\hline 2.5269 .422 & 0.3181246 & RR1-S & RR1-S & RR1-S & peak at $5.4496 ? ?$ \\
\hline 2.5271 .1466 & 0.3182645 & RR1-S & RR1-D & RR1-D & \\
\hline 2.5271 .1540 & 0.4394383 & RR1-PC & RR1-PC & RR1-PC & \\
\hline 2.5271 .255 & 0.4347510 & RR1-BL2 & RR1-BL2+PC & RR1-BL2+PC & $\Delta f= \pm 0.0012$ \\
\hline 2.5272 .1445 & 0.3137556 & RR1-S & RR1-S & RR1-S & \\
\hline 2.5272 .1454 & 0.3171863 & RR1-S & RR1-S & RR1-S & \\
\hline 2.5272 .238 & 0.3581800 & RR01 & RR01 & RR01 & \\
\hline 2.5273 .1267 & 0.4208925 & RR1-PC & RR1-PC & RR1-PC & wide, $\Delta f \approx \pm 0.0017$ \\
\hline 2.5273 .1356 & 0.3578176 & RR1-S & RR1-S & RR1-S & \\
\hline 2.5274 .1140 & 0.3437471 & RR1-S & RR1-S & RR1-S & \\
\hline 2.5274 .1185 & 0.3550667 & RR01 & RR01 & RR01 & \\
\hline 2.5388 .1150 & 0.3885822 & RR1-PC & RR1-PC & RR1-PC & wide \\
\hline 2.5389 .1138 & 0.3641254 & RR01 & RR01 & RR01 & \\
\hline 2.5389 .1478 & 0.2505581 & RR1-PC & RR1-PC & RR1-PC & $\Delta f=.00038$, symm., whitens out \\
\hline 2.5390 .844 & 0.3163344 & RR1-S & RR1-S & RR1-S & \\
\hline 2.5391 .1359 & 0.3512177 & RR1-S & RR1-S & RR1-S & \\
\hline 2.5394 .801 & 0.2870105 & RR1-S & RR1-S? & RR1-S & \\
\hline 2.5395 .855 & 0.2790402 & RR1-S & RR1-BL1 & RR1-PC & \\
\hline 2.5507 .6257 & 0.3679040 & RR1-S & RR1-S & RR1-S & \\
\hline 2.5510 .1080 & 0.3543775 & RR1-S & RR1-MI? & RR1-S & \\
\hline 2.5511 .772 & 0.3029241 & RR1-S & RR1-S & RR1-S & \\
\hline 2.5513 .821 & 0.3505221 & RR1-S & RR1-S & RR1-S & \\
\hline
\end{tabular}


Table 5. continued.

\begin{tabular}{|c|c|c|c|c|c|}
\hline$\overline{\mathrm{MACHO} \text { ID }}$ & $\begin{array}{c}\text { Period } \\
\text { [d] }\end{array}$ & $\begin{array}{l}\text { Type } r \\
\text { (A00) }\end{array}$ & $\begin{array}{c}\text { Type } r, b \\
\text { (SAM) } \\
6.5 \text { years }\end{array}$ & $\begin{array}{l}\text { Type } r, b \\
\text { (SAM) } \\
7.5 \text { years }\end{array}$ & 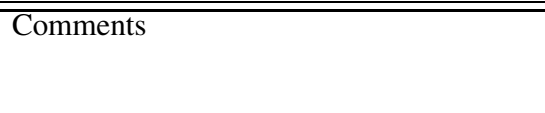 \\
\hline 2.5514 .660 & 0.3153734 & RR1-PC & RR1-PC & RR1-PC & \\
\hline 2.5516 .783 & 0.2615758 & RR1-S & RR1-S & RR1-S & \\
\hline 2.5628 .6071 & 0.2812632 & RR1-S & RR1-S & RR1-S & \\
\hline 2.5630 .1156 & 0.3123141 & RR1-PC & RR1-PC & RR1-BL2? & $\Delta f=.0004$, whitens out \\
\hline 2.5632 .1001 & 0.2771319 & RR1-S & RR1-S & RR1-S & \\
\hline 2.5632 .931 & 0.2861475 & RR1-S & RR1-S & RR1-S & \\
\hline 2.5633 .1275 & 0.2959713 & RR1-S & RR1-S & RR1-S & \\
\hline 2.5633 .1369 & 0.2937634 & RR1-S & RR1-S & RR1-S & peak at $\approx 2 v_{0}-2$ \\
\hline 2.5634 .989 & 0.3432276 & RR1-S & RR1-S & RR1-S & \\
\hline 2.5749 .6175 & 0.3255826 & RR1-S & RR1-S & RR1-S & \\
\hline 2.5750 .3309 & 0.3117014 & RR1-S & RR1-S & RR1-S & \\
\hline 2.5751 .1094 & 0.3407763 & RR1-S & RR1-S & RR1-S & \\
\hline 2.5751 .923 & 0.3233653 & RR1-S & RR1-PC & RR1-PC & \\
\hline 2.5752 .387 & 0.4592303 & RR1-S & RR1-S & RR1-S & peak at $\approx 4.6$ in $r$ only \\
\hline 2.5755 .839 & 0.3240553 & RR1-S & RR1-S & RR1-S & \\
\hline 2.5755 .874 & 0.3080512 & RR1-S & RR1-S & RR1-S & \\
\hline 2.5758 .661 & 0.2663870 & RR1-S & RR1-S & RR1-S & peak at $\approx 1.3$ in $r$ only \\
\hline 2.5870 .4598 & 0.3523722 & RR1-PC & RR1-PC & RR1-PC & very wide \\
\hline 2.5871 .3052 & 0.3144845 & RR1-S & RR1-S & RR1-D & \\
\hline 2.5872 .1272 & 0.3626709 & RR1-PC & RR1-PC & RR1-PC & \\
\hline 2.5873 .332 & 0.2964994 & RR1-S & RR1-S & RR1-S & \\
\hline 2.5875 .1085 & 0.3418996 & RR1-S & RR1-S & RR1-S & \\
\hline 2.5875 .250 & 0.3190485 & RR1-S & RR1-D & RR1-S & \\
\hline 2.5876 .444 & 0.3966802 & RR1-PC & RR1-PC & RR1-PC & very wide \\
\hline 2.5876 .741 & 0.2859854 & RR1-S & RR1-BL1 & RR1-BL1 & $\Delta f=-0.0722$ \\
\hline 3.6233 .503 & 0.3402755 & RR1-S & RR1-S & RR1-S & \\
\hline 3.6236 .690 & 0.4356755 & RR1-PC & RR1-PC & RR1-PC & \\
\hline 3.6238 .647 & 0.3389355 & RR1-S & RR1-S & RR1-S & \\
\hline 3.6240 .450 & 0.3836371 & RR1-BL2 & RR1-BL2 & RR1-BL2 & $+\mathrm{RR} 1-\mathrm{PC} \Delta f= \pm 0.0012$ \\
\hline 3.6240 .470 & 0.3349953 & RR1-PC & RR1-PC & RR1-PC & \\
\hline 3.6242 .544 & 0.2709690 & RR1-S & RR1-S & RR1-S & \\
\hline 3.6243 .353 & 0.2838777 & RR1-S & RR1-S & RR1-S & \\
\hline 3.6243 .404 & 0.2708506 & RR1-D & RR1-D & RR1-D & \\
\hline 3.6354 .459 & 0.3600521 & RR1-S & RR1-S & RR1-S & +weak RR1-D in $r$ crossID: 80.6354 .3448 \\
\hline 3.6356 .1136 & 0.3494046 & RR1-S & RR1-S & RR1-S & \\
\hline 3.6358 .619 & 0.3685809 & RR1-S & RR1-S & RR1-S & \\
\hline 3.6358 .924 & 0.2849506 & RR1-S & RR1-S & RR1-S & peak at $\approx 1.65$ in $b$ only \\
\hline 3.6359 .900 & 0.3110378 & RR1-S & RR1-S & RR1-S & \\
\hline 3.6360 .656 & 0.3545480 & RR1-PC & RR1-PC & RR1-PC & \\
\hline 3.6362 .689 & 0.3573751 & RR1-PC & RR1-PC & RR1-PC & \\
\hline 3.6362 .753 & 0.3584007 & RR1-S & RR1-MI? & RR1-S & \\
\hline 3.6362 .766 & 0.3297486 & RR1-S & RR1-S & RR1-S & \\
\hline 3.6363 .700 & 0.3403633 & RR1-S & RR1-S & RR1-S & \\
\hline 3.6364 .606 & 0.3419252 & RR1-S & RR1-S & RR1-S & \\
\hline 3.6364 .637 & 0.3623667 & RR1-S & RR1-S & RR1-S & RR1-MC in $b$ only!!!! \\
\hline 3.6481 .718 & 0.2745777 & RR1-S & RR1-S & RR1-S & \\
\hline 3.6482 .728 & 0.2954001 & RR1-S & RR1-S & RR1-S & \\
\hline 3.6597 .1167 & 0.3004253 & RR1-S & RR1-S & RR1-S & \\
\hline 3.6597 .756 & 0.3086451 & RR1-S & RR1-BL2 & RR1-BL2 & $\Delta f=0.00095$ crossID: 80.6597 .4435 \\
\hline 3.6598 .939 & 0.3678199 & RR1-PC & RR1-PC & RR1-PC & $\Delta f= \pm 0.0003$ \\
\hline 3.6602 .677 & 0.3454810 & RR1-S & RR1-S & RR1-S & \\
\hline 3.6602 .827 & 0.3298892 & RR1-S & RR1-S & RR1-S & \\
\hline 3.6603 .595 & 0.3439608 & RR1-S & RR1-S & RR1-S & \\
\hline 3.6603 .795 & 0.2765073 & RR1-BL1 & RR1-BL1 & RR1-BL1 & $\Delta f=-0.0173$ \\
\hline 3.6717 .628 & 0.3484935 & RR1-S & RR1-S & RR1-S & \\
\hline 3.6723 .769 & 0.3692349 & RR1-PC & RR1-PC & RR1-PC & \\
\hline 3.6724 .676 & 0.3413850 & RR1-S & RR1-S & RR1-S & \\
\hline 3.6725 .468 & 0.3333831 & RR1-S & RR1-S & RR1-S & \\
\hline 3.6725 .519 & 0.3567805 & RR01 & RR01 & RR01 & \\
\hline 3.6838 .1298 & 0.3583389 & RR1-PC & RR1-PC & RR1-BL2 & $\Delta f= \pm .0004$ crossID: 80.6838 .2884 \\
\hline 3.6839 .2073 & 0.3451022 & RR1-S & RR1-S & RR1-S & \\
\hline 3.6839.2292 & 0.3043062 & RR1-S & RR1-S & RR1-S & crossID: 80.6839 .4533 \\
\hline 3.6840 .1964 & 0.3332806 & RR1-S & RR1-S & RR1-S & \\
\hline 3.6840 .330 & 0.2803475 & RR1-S & RR1-S & RR1-S & \\
\hline 3.6841 .1739 & 0.3013309 & RR1-S & RR1-S & RR1-S & \\
\hline
\end{tabular}


Table 5. continued.

\begin{tabular}{|c|c|c|c|c|c|}
\hline MACHO ID & $\begin{array}{c}\text { Period } \\
\text { [d] }\end{array}$ & $\begin{array}{l}\text { Type } r \\
\text { (A00) }\end{array}$ & $\begin{array}{c}\text { Type } r, b \\
(\text { SAM) } \\
6.5 \text { years }\end{array}$ & $\begin{array}{l}\text { Type } r, b \\
\text { (SAM) } \\
7.5 \text { years }\end{array}$ & 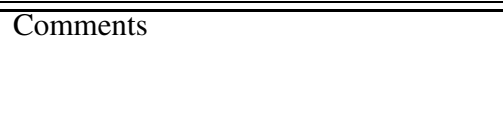 \\
\hline 3.6846 .1385 & 0.3324324 & RR1-S & RR1-S & RR1-S & \\
\hline 3.6847 .1380 & 0.2772299 & RR1-S & RR1-BL1? & RR1-S & + peak in $b$ \\
\hline 3.6848 .1045 & 0.2799079 & RR1-S & RR1-S & RR1-S & \\
\hline 3.6961 .824 & 0.3098935 & RR1-D & RR1-D & RR1-D & \\
\hline 3.6963 .574 & 0.3580682 & RR01 & RR01 & RR01 & \\
\hline 3.6964 .448 & 0.3300737 & RR1-S & RR1-S & RR1-S & \\
\hline 3.6964 .499 & 0.4028749 & RR1-S & RR1-S & RR1-S & \\
\hline 3.6964 .590 & 0.3820309 & RR1-S & RR1-S & RR1-S & RR1-D in $b$ \\
\hline 3.6964 .602 & 0.3347313 & RR1-S & RR1-S & RR1-S & \\
\hline 3.6965 .609 & 0.3261702 & RR1-S & RR1-S & RR1-S & \\
\hline 3.6966 .427 & 0.2972764 & RR1-S & RR1-BL1 & RR1-BL1 & \\
\hline 3.6966 .557 & 0.3334606 & RR1-S & RR1-S & RR1-S & \\
\hline 3.7082 .957 & 0.3574617 & RR01 & RR01 & RR01 & \\
\hline 3.7083 .609 & 0.3247032 & RR1-S & RR1-S & RR1-S & \\
\hline 3.7088 .623 & 0.3244180 & RR1-PC & RR1-PC & RR1-BL2 & $\Delta f= \pm .0006$, rem \\
\hline 3.7089 .446 & 0.3446722 & RR1-S & RR1-S & RR1-S & \\
\hline 3.7201 .504 & 0.3191092 & RR1-S & RR1-S & RR1-S & crossID: 80.7201 .3035 \\
\hline 3.7202 .916 & 0.3571132 & RR01 & RR01 & RR01 & crossID: 80.7202 .4678 \\
\hline 3.7203 .866 & 0.3669407 & RR01 & RR01 & RR01 & \\
\hline 3.7205 .727 & 0.3812379 & RR1-PC & RR1-PC & RR1-PC & \\
\hline 3.7208 .477 & 0.3645413 & RR01 & RR01 & RR01 & \\
\hline 3.7322 .614 & 0.3180069 & RR1-S & RR1-S & RR1-S & crossID: 80.7322 .3124 \\
\hline 3.7326 .897 & 0.2724766 & RR1-S & RR1-S & RR1-S & \\
\hline 3.7329 .399 & 0.3725990 & RR1-S & RR1-S & RR1-S & \\
\hline 3.7331 .413 & 0.2767823 & RR1-S & RR1-S & RR1-S & \\
\hline 3.7331 .467 & 0.3559271 & RR01 & RR01 & RR01 & \\
\hline 3.7332 .492 & 0.3495740 & RR1-S & RR1-S & RR1-S & \\
\hline 3.7444 .744 & 0.3419769 & RR1-S & RR1-S & RR1-S & \\
\hline 3.7444 .782 & 0.3174510 & RR1-PC & RR1-PC & RR1-PC & \\
\hline 3.7447 .739 & 0.3490642 & RR01 & RR01 & RR01 & \\
\hline 3.7448 .428 & 0.3485081 & RR01 & RR01 & RR01 & \\
\hline 3.7449 .447 & 0.3392843 & RR1-S & RR1-S & RR1-S & \\
\hline 3.7450 .214 & 0.3919072 & RR1-PC & RR1-PC & RR1-PC & \\
\hline 3.7451 .484 & 0.3025884 & RR1-S & RR1-S & RR1-MI & $v_{\mathrm{MI}}=0.8361 \cdot v_{0}$, in $b$ only \\
\hline 3.7452 .501 & 0.3837212 & RR1-S & RR1-S & RR1-S & \\
\hline 3.7452 .536 & 0.3432080 & RR1-S & RR1-S & RR1-S & \\
\hline 3.7453 .433 & 0.3402314 & RR1-S & RR1-S & RR1-S & \\
\hline 47.1401 .225 & 0.2825295 & RR1-S & RR1-S & RR1-D & in $b$ \\
\hline 47.1521 .589 & 0.3587236 & RR1-S & RR1-BL2 & RR1-BL2 & $\Delta f= \pm 0.0423$ \\
\hline 47.1526.209 & 0.3490908 & RR01 & RR01 & RR01 & \\
\hline 47.1528 .169 & 0.6626505 & RR1-S & RR1-PC & RR1-PC & \\
\hline 47.1529 .356 & 0.2723754 & RR1-S & RR1-S & RR1-S & \\
\hline 47.1529 .379 & 0.3181970 & RR1-S & RR1-S & RR1-S & \\
\hline 47.1530 .341 & 0.3530136 & RR1-S & RR1-S & RR1-S & \\
\hline 47.1531 .207 & 0.3347459 & RR1-S & RR1-S & RR1-S & \\
\hline 47.1643 .373 & 0.3402277 & RR1-S & RR1-S & RR1-S & \\
\hline 47.1645 .407 & 0.3191876 & RR1-S & RR1-S & RR1-PC & alias \\
\hline 47.1647.290 & 0.3496320 & RR01 & RR01 & RR01 & \\
\hline 47.1771 .401 & 0.3070320 & RR1-S & RR1-S & RR1-S & \\
\hline 47.1772 .308 & 0.3262692 & RR1-S & RR1-S & RR1-PC & one peak, whitens out, at the $\approx 1 / T$ lim. \\
\hline 47.1883 .161 & 0.3526035 & RR1-S & RR1-S & RR1-S & \\
\hline 47.2006 .1210 & 0.3474766 & RR1-S & RR1-S & RR1-S & \\
\hline 47.2013 .132 & 0.2881477 & RR1-S & RR1-S & RR1-S & \\
\hline 47.2126 .530 & 0.3588453 & RR1-S & RR1-S & RR1-S & \\
\hline 47.2130 .520 & 0.4049867 & RR1-S & RR1-S & RR1-S & alias at $v_{0}+2$ \\
\hline 47.2133 .446 & 0.3262074 & RR1-S & RR1-S & RR1-S & \\
\hline 47.2134 .462 & 0.3276747 & RR1-S & RR1-S & RR1-S & \\
\hline 47.2247 .648 & 0.3920761 & RR01 & RR01 & RR01 & \\
\hline 47.2254 .504 & 0.2794452 & RR1-S & RR1-S & RR1-S & peak at $\approx 3.1$ in $b$ only \\
\hline 47.2368 .572 & 0.3360793 & RR1-S & RR1-S & RR1-D & in $b$ \\
\hline 47.2609 .56 & 0.3573236 & RR1-BL1 & RR01+BL1 & $\mathrm{RR} 01+\mathrm{BL} 1$ & \\
\hline 47.2610 .799 & 0.2811866 & RR1-S & RR1-S & RR1-D & \\
\hline 47.2611 .1088 & 0.3520640 & RR1-S & RR1-S & RR1-D & \\
\hline 47.2611 .1176 & 0.3446541 & RR1-S & RR1-S & RR1-S & \\
\hline 47.2613 .1137 & 0.3619210 & RR1-S & RR1-S & RR1-S & \\
\hline
\end{tabular}


Table 5. continued.

\begin{tabular}{|c|c|c|c|c|c|}
\hline MACHO ID & $\begin{array}{c}\text { Period } \\
\text { [d] }\end{array}$ & $\begin{array}{l}\text { Type } r \\
\text { (A00) }\end{array}$ & $\begin{array}{l}\text { Type } r, b \\
(\text { SAM) } \\
6.5 \text { years }\end{array}$ & $\begin{array}{l}\text { Type } r, b \\
\text { (SAM) } \\
7.5 \text { years }\end{array}$ & 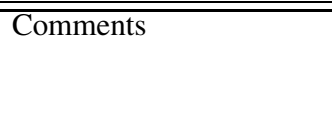 \\
\hline 47.2619 .1486 & 0.3658715 & RR01 & RR01 & RR01 & \\
\hline 5.4287 .1797 & 0.3434526 & RR1-S & RR1-S & RR1-S & \\
\hline 5.4400 .1081 & 0.3322317 & RR1-S & RR1-S & RR1-S & crossID: 10.4400 .4594 \\
\hline 5.4401 .1018 & 0.2685325 & RR1-BL2 & RR1-BL2 & RR1-BL2 & $\Delta f=0.0047$ \\
\hline 5.4401 .868 & 0.3530038 & RR01 & RR01 & RR01 & \\
\hline 5.4402 .1009 & 0.3261942 & RR1-S & RR1-S & RR1-S & crossID: 10.4402 .4727 \\
\hline 5.4407 .1348 & 0.3399189 & RR1-S & RR1-S & RR1-S & \\
\hline 5.4409 .5680 & 0.3521620 & RR1-S & RR1-S & RR1-S & \\
\hline 5.4409 .5985 & 0.3296998 & RR1-S & RR1-S & RR1-S & \\
\hline 5.4521 .1005 & 0.3591018 & RR1-S & RR1-S & RR1-S & \\
\hline 5.4521 .1330 & 0.3337677 & RR1-S & RR1-S & RR1-S & \\
\hline 5.4522 .1059 & 0.3589848 & RR01 & RR01 & RR01 & \\
\hline 5.4524 .1212 & 0.3461996 & RR01 & RR01 & RR01 & crossID: 10.4524 .4611 \\
\hline 5.4524 .972 & 0.3742322 & RR1-PC & RR1-PC & RR1-PC & \\
\hline 5.4526 .966 & 0.2813246 & RR1-S & RR1-S & RR1-S & \\
\hline 5.4528 .1555 & 0.2752904 & RR1-S & RR1-BL1 & RR1-BL1 & \\
\hline 5.4530 .5228 & 0.3448424 & RR1-S & RR1-S & RR1-S & \\
\hline 5.4531 .4873 & 0.2914121 & RR1-S & RR1-S & RR1-S & \\
\hline 5.4642 .1017 & 0.2989019 & RR1-S & RR1-BL1? & RR1-S & weak RR1-D \\
\hline 5.4642 .902 & 0.3641288 & RR1-S & RR1-S? & RR1-S & \\
\hline 5.4645 .1196 & 0.3982278 & RR1-S & RR1-S & RR1-S & \\
\hline 5.4646 .1080 & 0.2938281 & RR1-S & RR1-S & RR1-S & \\
\hline 5.4646 .1198 & 0.3220916 & RR1-S & RR1-S & RR1-D & +peak at $\approx 4.25$ in $b$ only \\
\hline 5.4649 .1029 & 0.3252660 & RR1-D & RR1-D & RR1-D & \\
\hline 5.4649 .1307 & 0.3293101 & RR1-S & RR1-S & RR1-S & \\
\hline 5.4765 .1125 & 0.2817559 & RR1-S & RR1-S & RR1-S & \\
\hline 5.4766 .1109 & 0.3324650 & RR1-PC & RR1-PC & RR1-PC & \\
\hline 5.4766 .1302 & 0.2736351 & RR1-S & RR1-S & RR1-S & \\
\hline 5.4766 .918 & 0.3610716 & RR1-PC & RR1-PC & RR1-PC & \\
\hline 5.4767 .1388 & 0.3078064 & RR1-PC & RR1-PC & RR1-PC & \\
\hline 5.4767 .952 & 0.3811693 & RR01 & RR01 & RR01 & \\
\hline 5.4767 .962 & 0.3630248 & RR01 & RR01 & RR01 & \\
\hline 5.4768 .1122 & 0.3356274 & RR1-S & RR1-S & RR1-S & \\
\hline 5.4769 .1287 & 0.3442608 & RR1-S & RR1-S & RR1-S & \\
\hline 5.4769 .1363 & 0.3785277 & RR1-PC & RR1-PC & RR1-PC & \\
\hline 5.4771 .2743 & 0.4003445 & RR1-S & RR1-S & RR1-S & \\
\hline 5.4772 .5496 & 0.3420781 & RR1-S & RR1-S & RR1-S & \\
\hline 5.4885 .1316 & 0.3253087 & RR1-S & RR1-BL1 & RR1-S & \\
\hline 5.4888 .796 & 0.3552391 & RR1-S & RR1-S & RR1-S & \\
\hline 5.4889 .1060 & 0.2815683 & RR1-S & RR1-BL1 & RR1-BL1 & in $b$ \\
\hline 5.4889 .1102 & 0.4627494 & RR01 & RR01 & RR01 & \\
\hline 5.4889 .921 & 0.3447723 & RR01 & RR01 & RR01 & \\
\hline 5.4891 .1617 & 0.3217797 & RR1-PC & RR1-PC & RR1-PC & \\
\hline 5.4892 .3500 & 0.3581734 & RR01 & RR01 & RR01 & \\
\hline 5.4893 .6682 & 0.2927763 & RR1-S & DD1-D & RR1-S & weak RR1-D \\
\hline 5.5008 .1556 & 0.3866424 & RR1-S & RR1-S & RR1-PC & \\
\hline 5.5008 .1902 & 0.3774283 & RR1-PC & $\mathrm{RR} 1-\mathrm{PC}+\mathrm{BL} 1$ & $\mathrm{RR} 1-\mathrm{PC}+\mathrm{BL} 1$ & \\
\hline 5.5011 .1801 & 0.2946025 & RR1-S & RR1-S & RR1-S & weak RR1-D \\
\hline 5.5013 .3494 & 0.3504694 & RR01 & RR01 & RR01 & \\
\hline 5.5126 .1055 & 0.3388227 & RR1-S & RR1-S & RR1-S & \\
\hline 5.5128 .1262 & 0.4279750 & RR1-MC & RR1-MC & RR1-MC & $\Delta f_{1}=\Delta f_{2}=-.0010$ \\
\hline 5.5128 .1322 & 0.3821018 & RR1-S & RR1-S & RR1-S & \\
\hline 5.5129 .1381 & 0.2687570 & RR1-S & RR1-S & RR1-S & \\
\hline 5.5129 .1451 & 0.2899344 & RR1-S & RR1-S & RR1-D & \\
\hline 5.5130 .1099 & 0.3004342 & RR1-S & RR1-S & RR1-S & \\
\hline 5.5131 .1076 & 0.3227172 & RR1-S & RR1-S & RR1-S & \\
\hline 5.5131 .1128 & 0.3154071 & RR1-S & RR1-S & RR1-S & \\
\hline 5.5131 .954 & 0.3576141 & RR1-S & RR1-S & RR1-S & + peak in $r$ only at $\approx 1.2$ \\
\hline 5.5132 .2100 & 0.3370932 & RR1-S & RR1-S & RR1-S & \\
\hline 5.5134 .3483 & 0.3473474 & RR1-S & RR1-S & RR1-S & \\
\hline 5.5134 .3773 & 0.3309423 & RR1-S & RR1-S & RR1-S & \\
\hline 5.5136 .5295 & 0.3678665 & RR01 & RR01 & RR01 & \\
\hline 5.5247 .894 & 0.3732139 & RR01 & RR01 & RR01 & \\
\hline 5.5249 .1034 & 0.3393207 & RR1-S & RR1-D & RR1-D & \\
\hline 5.5250 .1199 & 0.3574901 & RR1-S & RR1-S & RR1-S & \\
\hline
\end{tabular}


Table 5. continued.

\begin{tabular}{|c|c|c|c|c|c|}
\hline$\overline{\mathrm{MACHO} \text { ID }}$ & $\begin{array}{c}\text { Period } \\
\text { [d] }\end{array}$ & $\begin{array}{l}\text { Type } r \\
\text { (A00) }\end{array}$ & $\begin{array}{l}\text { Type } r, b \\
(\text { SAM) } \\
6.5 \text { years }\end{array}$ & $\begin{array}{l}\text { Type } r, b \\
\text { (SAM) } \\
7.5 \text { years }\end{array}$ & 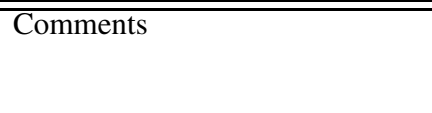 \\
\hline 5.5250 .1501 & 0.3551757 & $\overline{R R 1-P C}$ & RR1-PC & RR1-BL2 & symm. $\Delta f= \pm .0005$, whitens out \\
\hline 5.5251 .1187 & 0.3414968 & RR1-S & RR1-S & RR1-S & \\
\hline 5.5251 .1298 & 0.3171333 & RR1-S & RR1-S & RR1-S & \\
\hline 5.5251 .1676 & 0.3375434 & RR1-S & RR1-S & RR1-S & \\
\hline 5.5252 .708 & 0.3663170 & RR1-S & RR1-S & RR1-PC & \\
\hline 5.5252 .890 & 0.3555672 & RR1-S & RR1-S & RR1-S & \\
\hline 5.5253 .953 & 0.2853174 & RR1-S & RR1-S & RR1-S & \\
\hline 5.5254 .1692 & 0.3453603 & RR01 & RR01 & RR01 & \\
\hline 5.5367 .3432 & 0.2854189 & RR1-S & RR1-MC & RR1-BL2 & $\Delta f= \pm 0.0296\left(\right.$ no $\left.\Delta v_{2}\right)$ \\
\hline 5.5368 .1201 & 0.3365130 & RR1-BL2 & RR1-BL2 & RR1-BL2 & $\Delta f= \pm 0.0056$ \\
\hline 5.5369 .1184 & 0.3837979 & RR01 & RR01 & RR01 & \\
\hline 5.5370 .1059 & 0.3522415 & RR1-S & RR1-S & RR1-S & \\
\hline 5.5371 .1247 & 0.3111854 & RR1-S & RR1-S & RR1-D & \\
\hline 5.5371 .1493 & 0.3114060 & RR1-S & RR1-S & RR1-D & in $r$ \\
\hline 5.5372 .1172 & 0.3322713 & RR1-S & RR1-S & RR1-S & weak RR1-D \\
\hline 5.5372 .1429 & 0.3471178 & RR01 & RR01 & RR01 & \\
\hline 5.5374 .1149 & 0.3177985 & RR1-S & RR1-S & RR1-S & \\
\hline 5.5375 .1852 & 0.3130981 & RR1-S & RR1-S & RR1-S & \\
\hline 5.5489 .1125 & 0.3898567 & RR1-PC & RR1-PC & RR1-PC & \\
\hline 5.5489 .1188 & 0.2920376 & RR1-S & RR1-PC & RR1-PC & \\
\hline 5.5489 .1289 & 0.3619541 & RR01 & RR01 & RR01 & \\
\hline 5.5489 .1397 & 0.2899676 & RR1-BL1 & RR1-BL1 & RR1-BL1 & $\Delta f=-0.0478$ \\
\hline 5.5490 .1390 & 0.2935354 & RR1-S & RR1-S & RR1-S & \\
\hline 5.5492 .1293 & 0.3943940 & RR01 & RR01 & RR01 & \\
\hline 5.5492 .1482 & 0.3337507 & RR1-S & RR1-S & RR1-S & \\
\hline 5.5492 .1489 & 0.3506307 & RR1-S & RR1-S & RR1-D & \\
\hline 5.5492 .1619 & 0.2661855 & RR1-S & RR1-D & RR1-S & \\
\hline 5.5493 .1455 & 0.2964112 & RR1-S & RR1-S & RR1-S & \\
\hline 5.5495 .1326 & 0.3687091 & RR1-S & RR1-S & RR1-S & \\
\hline 5.5496 .1582 & 0.3454742 & RR1-S & RR1-S & RR1-S & \\
\hline 5.5496 .2445 & 0.2885515 & RR1-S & RR1-S & RR1-S & \\
\hline 5.5497 .3874 & 0.2680527 & RR1-S & RR1-BL1 & RR1-BL1 & "+" is very weak \\
\hline 5.5610 .2922 & 0.3259662 & RR1-S & RR1-D & RR1-D & mostly in $b$ \\
\hline 5.5611 .2835 & 0.2944371 & RR1-S & RR1-PC & RR1-PC & \\
\hline 5.5612 .2886 & 0.3159385 & RR1-S & RR1-S & RR1-S & \\
\hline 5.5614 .3014 & 0.3327409 & RR1-S & RR1-S & RR1-S & \\
\hline 6.5721 .289 & 0.3355822 & RR1-S & RR1-S & RR1-S & crossID: 13.5721 .2108 \\
\hline 6.5721 .310 & 0.2974560 & RR1-S & RR1-S & RR1-S & crossID: 13.5721 .2163 \\
\hline 6.5721 .352 & 0.3766420 & RR01 & RR01 & RR01 & crossID: 13.5721 .2290 \\
\hline 6.5722 .682 & 0.2944694 & RR1-S & RR1-S & RR1-S & crossID: 13.5722 .3565 \\
\hline 6.5724 .620 & 0.3829355 & RR1-PC & RR1-PC & RR1-PC & \\
\hline 6.5724 .869 & 0.3340855 & RR1-S & RR1-S & RR1-S & \\
\hline 6.5724 .887 & 0.3019672 & RR1-S & RR1-S & RR1-S & \\
\hline 6.5727 .790 & 0.2684920 & RR1-S & RR1-S & RR1-D & \\
\hline 6.5728 .1012 & 0.3413199 & RR1-S & RR1-S & RR1-S & \\
\hline 6.5728 .873 & 0.2709565 & RR1-S & RR1-S & RR1-S & \\
\hline 6.5729 .1008 & 0.3501063 & RR1-PC & RR1-PC & RR1-PC & \\
\hline 6.5729 .958 & 0.2778179 & RR1-BL1 & RR1-BL1+MI & RR1-BL1 & $\Delta f=+0.0742$ \\
\hline 6.5730 .3852 & 0.3986377 & RR1-S & RR1-S & RR1-S & \\
\hline 6.5730 .3869 & 0.3551622 & RR01 & RR01 & RR01 & \\
\hline 6.5730 .4053 & 0.3463310 & RR1-S & RR1-S & RR1-S & \\
\hline 6.5730 .4057 & 0.2763214 & RR1-BL1 & RR1-BL1 & RR1-BL1 & $\Delta f=+0.0776$ \\
\hline 6.5731 .4354 & 0.2412002 & RR1-S & RR1-S & RR1-S & \\
\hline 6.5732 .3906 & 0.3523542 & RR01 & RR01 & RR01 & \\
\hline 6.5842 .396 & 0.3604255 & RR01 & RR01 & RR01 & crossID: 13.5842 .2316 \\
\hline 6.5844 .872 & 0.3702682 & RR1-PC & RR1-PC & RR1-PC & crossID: 13.5844 .3963 \\
\hline 6.5846 .967 & 0.3394754 & RR1-S & RR1-S & RR1-S & peak in $r$ at $\approx 2 v_{0}$ \\
\hline 6.5847 .252 & 0.2890379 & RR1-S & RR1-D & RR1-D & \\
\hline 6.5848 .1122 & 0.3591429 & RR1-PC & RR1-PC & RR1-PC & \\
\hline 6.5849 .1114 & 0.2553474 & RR1-S & RR1-S & RR1-S & \\
\hline 6.5849 .1135 & 0.3132256 & RR1-S & RR1-PC & RR1-PC & \\
\hline 6.5850 .1081 & 0.3335893 & RR1-S & RR1-S & RR1-BL1 & $\Delta f=-0.2283$ \\
\hline 6.5850 .1195 & 0.3315815 & RR1-S & RR1-S & RR1-S & \\
\hline 6.5850 .1436 & 0.2720254 & RR1-S & RR1-S & RR1-S & \\
\hline 6.5850 .933 & 0.3730655 & RR01 & RR01 & RR01 & \\
\hline
\end{tabular}


Table 5. continued.

\begin{tabular}{|c|c|c|c|c|c|}
\hline MACHO ID & $\begin{array}{c}\text { Period } \\
\text { [d] }\end{array}$ & $\begin{array}{l}\text { Type } r \\
\text { (A00) }\end{array}$ & $\begin{array}{c}\text { Type } r, b \\
(\text { SAM) } \\
6.5 \text { years }\end{array}$ & $\begin{array}{l}\text { Type } r, b \\
\text { (SAM) } \\
7.5 \text { years }\end{array}$ & Comments \\
\hline 6.5851 .3773 & 0.3209512 & RR1-S & RR1-S & RR1-S & \\
\hline 6.5852 .4852 & 0.3431204 & RR1-S & RR1-S & RR1-S & peak in $b$ at $\approx 2 v_{0}$ \\
\hline 6.5852 .4915 & 0.3562610 & RR1-S & RR1-S & RR1-S & \\
\hline 6.5852 .5312 & 0.3057469 & RR1-S & RR1-S & RR1-S & \\
\hline 6.5853 .3986 & 0.3554134 & RR01 & RR01 & RR01 & \\
\hline 6.5963 .363 & 0.2764715 & RR1-S & RR1-S & RR1-S & crossID: 13.5963 .2479 \\
\hline 6.5965 .835 & 0.3169592 & RR1-S & RR1-S & RR1-S & crossID: 13.5965 .3725 \\
\hline 6.5965 .890 & 0.3669381 & RR1-S & RR1-S & RR1-D & mostly in $b$; crossID: 13.5965 .3700 \\
\hline 6.5966 .757 & 0.3132610 & RR1-S & RR1-S & RR1-S & \\
\hline 6.5966 .886 & 0.3393764 & RR1-S & RR1-S & RR1-S & \\
\hline 6.5968 .1603 & 0.3851853 & RR1-S & RR1-S & RR1-S & \\
\hline 6.5971 .1194 & 0.4009782 & RR1-PC & RR1-PC & RR1-PC & \\
\hline 6.5971 .1233 & 0.2877179 & RR1-BL2 & RR1-BL2 & RR1-BL2 & $\Delta f= \pm 0.0735+\mathrm{al} .2 v_{0}+\mathrm{fm}$ \\
\hline 6.5971 .1408 & 0.3712570 & RR01 & RR01 & RR01 & \\
\hline 6.5972 .5021 & 0.2826945 & RR1-S & RR1-S & RR1-S & \\
\hline 6.5974 .3945 & 0.3725992 & RR1-S & RR1-S & RR1-D & +strange stuff in $b$ \\
\hline 6.6084 .462 & 0.3067477 & RR1-S & RR1-S & RR1-S & crossID: 13.6084 .2519 \\
\hline 6.6085 .884 & 0.3195912 & RR1-S & RR1-S & RR1-S & \\
\hline 6.6086 .792 & 0.3480795 & RR01 & RR01 & RR01 & \\
\hline 6.6087 .966 & 0.2831662 & RR1-S & RR1-S & RR1-PC & \\
\hline 6.6089 .1661 & 0.3591991 & RR01 & RR01 & RR01 & \\
\hline 6.6089 .1737 & 0.3477483 & RR1-S & RR1-S & RR1-S & \\
\hline 6.6091 .1198 & 0.2668322 & RR1-S & RR1-BL1 & RR1-BL1 & $\Delta f= \pm 0.0754$, “ + " is weak \\
\hline 6.6091 .1245 & 0.3474788 & RR1-S & RR1-S & RR1-S & + strange peak in $r$ \\
\hline 6.6091 .877 & 0.3206106 & RR1-BL1 & RR1-BL1 & RR1-BL1 & $\Delta f=-0.0385$ \\
\hline 6.6092 .1311 & 0.3547291 & RR1-S & RR1-S & RR1-S & + strange stuff in $b$ \\
\hline 6.6093 .5030 & 0.2653642 & RR1-D & RR1-D & RR1-D & $\mathrm{P}(\mathrm{A} 00) \neq \mathrm{P}(\mathrm{SAM})$ \\
\hline 6.6094 .5331 & 0.3356851 & RR1-PC & RR1-PC & RR1-PC & \\
\hline 6.6094 .5406 & 0.2851642 & RR1-PC & RR1-PC & RR1-PC & \\
\hline 6.6094 .5606 & 0.3393854 & RR1-PC & RR1-BL2 & RR1-BL2 & $\Delta f= \pm 0.0012 ?(+$ very weak RR1-PC) \\
\hline 6.6208 .869 & 0.3321579 & RR1-S & RR1-S & RR1-S & peak in $r$ at $\approx 1.82$ \\
\hline 6.6209 .727 & 0.2718806 & RR1-S & RR1-S & RR1-S & \\
\hline 6.6210 .1695 & 0.3391681 & RR1-S & RR1-S & RR1-S & \\
\hline 6.6210 .1712 & 0.3189901 & RR1-S & RR1-S & RR1-S & weak RR1-D in $r$ \\
\hline 6.6212 .1063 & 0.3602799 & RR01 & RR01 & RR01 & \\
\hline 6.6212 .1142 & 0.3732319 & RR1-PC & RR1-PC & RR1-PC & \\
\hline 6.6212 .1454 & 0.2820694 & RR1-S & RR1-S & RR1-S & \\
\hline 6.6213 .1506 & 0.3374679 & RR1-S & RR1-S & RR1-S & \\
\hline 6.6213 .1520 & 0.3156754 & RR1-S & RR1-S & RR1-S & RR1-D in $b$ \\
\hline 6.6213 .1685 & 0.3050434 & RR1-S & RR1-S & RR1-S & \\
\hline 6.6214 .4637 & 0.3491769 & RR01 & RR01 & RR01 & \\
\hline 6.6214 .5338 & 0.2819035 & RR1-S & RR1-S & RR1-S & \\
\hline 6.6215 .5777 & 0.2848354 & RR1-S & RR1-S & RR1-S & \\
\hline 6.6215 .6520 & 0.2787094 & RR1-S & RR1-S & RR1-S & \\
\hline 6.6326 .424 & 0.3304519 & RR1-BL1 & RR1-BL1 & $\mathrm{RR} 1-\mathrm{BL} 1+\mathrm{PC}$ & $\Delta f=-0.0726$, crossID: 13.6326 .2765 \\
\hline 6.6329 .884 & 0.3597879 & RR01 & RR01 & RR01 & \\
\hline 6.6331 .238 & 0.3448059 & RR1-S & RR1-S & RR1-S & \\
\hline 6.6333 .912 & 0.3483025 & RR1-S & RR1-S & RR1-S & \\
\hline 6.6335 .5311 & 0.3377354 & RR1-S & RR1-S & RR1-S & \\
\hline 6.6336 .6017 & 0.3565227 & RR1-S & RR1-S & RR1-S & \\
\hline 6.6447 .699 & 0.3501959 & RR1-S & RR1-S & RR1-PC & alias, crossID: 13.6447 .3202 \\
\hline 6.6448 .801 & 0.3431581 & RR1-S & RR1-S & RR1-S & stuff at 1.54 , crossID: 13.6448 .4137 \\
\hline 6.6450 .888 & 0.3437111 & RR1-S & RR1-D & RR1-D & in $b$ \\
\hline 6.6451 .919 & 0.4020766 & RR1-PC & RR1-PC+MC? & RR1-PC & wide \\
\hline 6.6452 .2394 & 0.3348319 & RR1-PC & RR1-PC & RR1-PC & \\
\hline 6.6452 .704 & 0.3540965 & RR01 & RR01 & RR01 & \\
\hline 6.6452 .811 & 0.3617118 & BI & BI & $\mathrm{BI}$ & light curve $\approx$ sinusoidal, $v_{1} / v_{0}=1 / 2$ \\
\hline 6.6453 .1387 & 0.2819384 & RR1-S & RR1-S & RR1-PC & \\
\hline 6.6455 .1326 & 0.3356315 & RR1-PC & RR1-PC & RR1-PC & \\
\hline 6.6455 .1877 & 0.2962379 & RR1-S & RR1-D & RR1-D & \\
\hline 6.6456 .5690 & 0.2941663 & RR1-S & RR1-S & RR1-S & \\
\hline 6.6457 .6650 & 0.3186543 & RR1-S & RR1-D & RR1-D & in $r$ \\
\hline 6.6568 .484 & 0.3380989 & RR1-PC & RR1-PC & RR1-PC & crossID: 13.6568 .3005 \\
\hline 6.6569 .955 & 0.3150184 & RR1-S & RR1-S & RR1-S & crossID: 13.6569 .4284 \\
\hline 6.6569 .980 & 0.3432539 & RR1-S & RR1-D & RR1-D & in $b$, crossID: 13.6569 .4088 \\
\hline
\end{tabular}


Table 5. continued.

\begin{tabular}{|c|c|c|c|c|c|}
\hline MACHO ID & $\begin{array}{c}\text { Period } \\
\text { [d] }\end{array}$ & $\begin{array}{l}\text { Type } r \\
\text { (A00) }\end{array}$ & $\begin{array}{l}\text { Type } r, b \\
\text { (SAM) } \\
6.5 \text { years }\end{array}$ & $\begin{array}{l}\text { Type } r, b \\
\text { (SAM) } \\
7.5 \text { years }\end{array}$ & 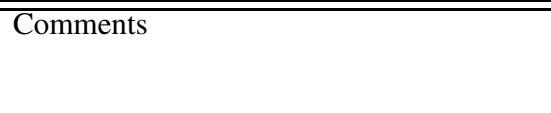 \\
\hline 6.6570 .751 & 0.3502577 & RR1-S & RR1-S & RR1-S & \\
\hline 6.6571 .851 & 0.2717850 & RR1-S & RR1-S & RR1-S & \\
\hline 6.6572 .919 & 0.3756908 & RR01 & RR01 & RR01 & \\
\hline 6.6574 .1353 & 0.3585347 & RR01 & RR01 & RR01 & \\
\hline 6.6576 .1701 & 0.3082586 & RR1-S & RR1-S & RR1-S & \\
\hline 6.6576 .558 & 0.2692476 & RR1-S & RR1-D & RR1-D & \\
\hline 6.6689 .563 & 0.3050574 & RR1-S & RR1-S & RR1-D & weak $v_{2}$ in $b$, crossID: 13.6689 .3055 \\
\hline 6.6690 .945 & 0.3417750 & RR1-S & RR1-S & RR1-D & \\
\hline 6.6692 .937 & 0.3568799 & RR1-PC & RR1-PC & RR1-PC & \\
\hline 6.6693 .1075 & 0.2963217 & RR1-S & RR1-S & RR1-S & \\
\hline 6.6697 .1361 & 0.3973327 & RR1-PC & RR1-PC & RR1-PC & very wide \\
\hline 6.6697 .1565 & 0.4334935 & RR1-MC & RR1-MC & RR1-MC & $\Delta v_{1} \pm=.00124, \Delta v_{2}= \pm .0005$ \\
\hline 6.6697 .1859 & 0.3072472 & RR1-S & RR1-S & RR1-S & \\
\hline 6.6699 .5598 & 0.3526863 & RR1-D & RR1-D & RR1-D & \\
\hline 6.6699 .5619 & 0.3304007 & RR1-S & RR1-S & RR1-S & \\
\hline 6.6699 .6734 & 0.2915436 & RR1-S & RR1-S & RR1-S & \\
\hline 6.6810 .428 & 0.3814159 & RR01 & RR01 & RR01 & crossID: 13.6810 .2845 \\
\hline 6.6810 .616 & 0.2883850 & RR1-PC & RR1-PC & RR1-BL2 & $\Delta f= \pm .0004$, wh., crossID: 13.6810 .2981 \\
\hline 6.6811 .481 & 0.3559612 & RR1-S & RR1-S & RR1-S & \\
\hline 6.6811 .651 & 0.3598909 & RR01 & RR01 & RR01 & crossID: 13.6811 .4041 \\
\hline 6.6812 .1063 & 0.3165557 & RR1-S & RR1-S & RR1-S & \\
\hline 6.6812 .923 & 0.3547845 & RR1-PC & RR1-PC & RR1-MC & \\
\hline 6.6812 .929 & 0.3212383 & RR1-S & RR1-S & RR1-S & +weak RR1-D \\
\hline 6.6813 .699 & 0.3825721 & RR1-PC & RR1-PC & RR1-PC & \\
\hline 6.6813 .807 & 0.2688031 & RR1-S & RR1-S & RR1-S & \\
\hline 6.6814 .901 & 0.2999740 & RR1-S & RR1-S & RR1-S & \\
\hline 6.6815 .747 & 0.2920236 & RR1-D & RR1-D & RR1-D & \\
\hline 6.6815 .788 & 0.3260021 & RR1-S & RR1-S & RR1-S & \\
\hline 6.6819 .6629 & 0.3393739 & RR1-S & RR1-S & RR1-S & \\
\hline 6.6819 .6763 & 0.2843131 & RR1-S & RR1-PC & RR1-PC & \\
\hline 6.6819 .7278 & 0.2845135 & RR1-S & RR1-D & RR1-D & \\
\hline 6.6820 .7288 & 0.2882788 & RR1-S & RR1-S & RR1-S & \\
\hline 6.6820 .7412 & 0.2948450 & RR1-S & RR1-S & RR1-S & \\
\hline 6.6820 .7594 & 0.3178720 & RR1-S & RR1-S & RR1-S & \\
\hline 6.6931 .649 & 0.3228060 & RR1-D & RR1-D & RR1-D & crossID: 13.6931 .3278 \\
\hline 6.6931 .655 & 0.3154455 & RR1-S & RR1-S & RR1-S & +weak RR1-D in $b$, crossID: 13.6931 .3219 \\
\hline 6.6931 .704 & 0.3590484 & RR1-S & RR1-S & RR1-S & crossID: 13.6931 .3165 \\
\hline 6.6933 .1043 & 0.3228416 & RR1-PC & RR1-PC & RR1-PC & \\
\hline 6.6933 .939 & 0.3454407 & RR01 & RR01 & RR01 & \\
\hline 6.6934 .1056 & 0.3376670 & RR1-S & RR1-S & RR1-S & \\
\hline 6.6936 .2825 & 0.2737831 & RR1-S & RR1-S & RR1-S & \\
\hline 6.6937 .1264 & 0.3655773 & RR1-S & RR1-S & RR1-S & \\
\hline 6.6937 .1923 & 0.3348225 & RR1-S & RR1-S & RR1-S & \\
\hline 6.6938 .1574 & 0.3219262 & RR1-S & RR1-S & RR1-S & \\
\hline 6.6938 .1698 & 0.3392113 & RR1-S & RR1-D & RR1-D & \\
\hline 6.6938 .1809 & 0.3432712 & RR1-S & RR1-S & RR1-S & \\
\hline 6.7054 .710 & 0.3129435 & RR1-S & RR1-S & RR1-S & crossID: 13.7054 .3006 \\
\hline 6.7054 .713 & 0.4404073 & RR1-BL2 & RR1-BL2 & RR1-BL2+PC & $\Delta f= \pm 0.0016$ \\
\hline 6.7055 .814 & 0.3506573 & RR1-S & RR1-S & RR1-S & \\
\hline 6.7056 .785 & 0.3492702 & RR1-S & RR1-S & RR1-S & \\
\hline 6.7056 .836 & 0.4476160 & RR1-S & RR1-D & RR1-BL2 & $\Delta f= \pm 0.0011,+$ weak RR1-D \\
\hline 6.7056 .952 & 0.3344727 & RR1-S & RR1-S & RR1-S & \\
\hline 6.7058 .1128 & 0.3244414 & RR1-S & RR1-S & RR1-S & \\
\hline 6.7059 .995 & 0.3156189 & RR1-S & RR1-S & RR1-S & \\
\hline 80.6231 .667 & 0.3557524 & RR1-S & RR1-S & RR1-S & \\
\hline 80.6233 .2102 & 0.2673977 & RR1-S & RR1-D & RR1-D & in $b,+$ peak at .4017 in $b$ \\
\hline 80.6345 .7307 & 0.2941656 & RR1-S & RR1-D & RR1-D & $v_{1}=2.00$ \\
\hline 80.6346 .2144 & 0.4649030 & RR01 & RR01 & RR01 & $v_{1}>v_{0}$ \\
\hline 80.6348 .1413 & 0.3046775 & RR1-S & RR1-D? & RR1-D & in $b$ \\
\hline 80.6348 .1828 & 0.3564758 & RR1-S & RR1-S & RR1-S & \\
\hline 80.6348 .2470 & 0.3381670 & RR1-S & RR1-S & RR1-MI & peak in $b$ at 1.2 \\
\hline 80.6349 .2365 & 0.3394050 & RR1-S & RR1-S & RR1-S & double peak at $\approx 2 v_{0}$ \\
\hline 80.6350 .1072 & 0.3346674 & RR1-S & RR1-D & RR1-D & \\
\hline 80.6350 .3508 & 0.3900628 & RR1-PC & RR1-PC & RR1-PC & \\
\hline 80.6351 .2358 & 0.3393575 & RR1-S & RR1-S & RR1-S & \\
\hline
\end{tabular}


Table 5. continued.

\begin{tabular}{|c|c|c|c|c|c|}
\hline$\overline{\text { MACHO ID }}$ & $\begin{array}{c}\text { Period } \\
\text { [d] }\end{array}$ & $\begin{array}{l}\text { Type } r \\
\text { (A00) }\end{array}$ & $\begin{array}{l}\text { Type } r, b \\
(\text { SAM) } \\
5 \text { years }\end{array}$ & $\begin{array}{l}\text { Type } r, b \\
\text { (SAM) } \\
7.5 \text { years }\end{array}$ & 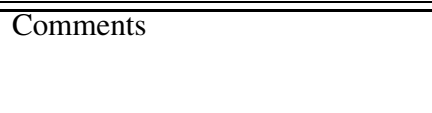 \\
\hline 80.6352 .1495 & 0.3079889 & RR1-BL1 & RR1-BL1 & RR1-BL1+PC & both are changing \\
\hline 80.6353 .1048 & 0.3071428 & RR1-S & RR1-S & RR1-D & in $b$ \\
\hline 80.6353 .1458 & 0.2753242 & RR1-D & RR1-D & RR1-D & \\
\hline 80.6354 .3448 & 0.3600498 & RR1-S & RR1-S & RR1-D & crossID: 3.6354 .459 \\
\hline 80.6354 .3658 & 0.3242243 & RR1-S & RR1-BL1? & RR1-S & \\
\hline 80.6355 .5162 & 0.3444493 & RR1-S & RR1-S & RR1-S & \\
\hline 80.6468 .1883 & 0.3346678 & RR1-S & RR1-S & RR1-S & \\
\hline 80.6468 .2765 & 0.3493692 & RR1-PC & RR1-PC & RR1-PC & wide \\
\hline 80.6469 .2447 & 0.3314219 & RR1-S & RR1-D & RR1-D & in $b$ \\
\hline 80.6470 .2000 & 0.3706713 & RR1-S & RR1-PC & RR1-BL2 & \\
\hline 80.6472 .1694 & 0.3284227 & RR1-S & RR1-S & RR1-S & \\
\hline 80.6475 .3548 & 0.3266714 & RR1-S & RR1-S & RR1-S & \\
\hline 80.6589 .1879 & 0.2499859 & RR1-S & RR1-D & RR1-S & weak RR1-D in $b$ \\
\hline 80.6589 .2425 & 0.3497221 & RR1-S & RR1-S & RR1-S & \\
\hline 80.6590 .1844 & 0.3511759 & RR01 & RR01 & RR01 & \\
\hline 80.6590 .1980 & 0.3453431 & RR1-S & RR1-S & RR1-S & \\
\hline 80.6590 .2058 & 0.3138516 & RR1-S & RR1-S & RR1-S & \\
\hline 80.6591 .2357 & 0.3345161 & RR1-S & RR1-S & RR1-S & \\
\hline 80.6595 .1558 & 0.3412933 & RR1-S & RR1-S & RR1-S & \\
\hline 80.6595 .1599 & 0.3323705 & RR1-BL2 & RR1-BL2 & RR1-BL2 & $\Delta f= \pm 0.0368$ \\
\hline 80.6596 .3127 & 0.2965595 & RR1-S & RR1-S & RR1-PC & \\
\hline 80.6597 .4435 & 0.3086445 & RR1-PC & RR1-BL1 & RR1-BL2 & crossID: 3.6597 .756 \\
\hline 80.6597 .4461 & 0.2635343 & RR1-S & RR1-S & RR1-S & \\
\hline 80.6597 .4703 & 0.3001928 & RR1-S & RR1-PC & RR1-S & \\
\hline 80.6708 .6771 & 0.2860939 & RR1-D & RR1-D & RR1-D & \\
\hline 80.6708 .6879 & 0.3139923 & RR1-S & RR1-D & RR1-D & \\
\hline 80.6709 .2322 & 0.3325378 & RR1-D & RR1-D & RR1-S & weak RR1-D in $r$ \\
\hline 80.6710 .2075 & 0.3478087 & RR1-S & RR1-D & RR1-S & \\
\hline 80.6712 .1521 & 0.3038647 & RR1-S & RR1-S & RR1-S & weak RR1-D in $r$ \\
\hline 80.6712 .1806 & 0.3365525 & RR1-S & RR1-S & RR1-S & \\
\hline 80.6830 .2303 & 0.3676399 & RR01 & RR01 & RR01 & \\
\hline 80.6832 .2030 & 0.2843896 & RR1-S & RR1-S & RR1-S & \\
\hline 80.6835 .1144 & 0.3410573 & RR1-S & RR1-S & RR1-D & \\
\hline 80.6835 .1220 & 0.3502291 & RR1-PC & RR1-PC & RR1-PC & \\
\hline 80.6835 .1442 & 0.3651713 & RR1-S & RR1-S & RR1-PC? & weak \\
\hline 80.6836 .1492 & 0.3720830 & RR01 & RR01 & RR01 & \\
\hline 80.6837 .1444 & 0.3257803 & RR1-S & RR1-D & RR1-D & in $r$ \\
\hline 80.6838 .2884 & 0.3583390 & RR1-PC & RR1-PC & RR1-BL2 & symmetric, crossID: 3.6838 .1298 \\
\hline 80.6839 .4533 & 0.3043054 & RR1-S & RR1-S & RR1-S & crossID: 3.6839 .2292 \\
\hline 80.6950 .6196 & 0.3021638 & RR1-S & RR1-D & RR1-D & \\
\hline 80.6950 .6414 & 0.3384454 & RR1-PC & RR1-PC & RR1-PC & \\
\hline 80.6950 .6751 & 0.2778871 & RR1-S & RR1-S & RR1-D & in $r$ \\
\hline 80.6951 .2395 & 0.3114197 & RR1-PC & RR1-PC & RR1-BL2 & symmetric \\
\hline 80.6953 .1499 & 0.3061991 & RR1-S & RR1-D & RR1-D & \\
\hline 80.6953 .1590 & 0.4061294 & RR1-PC & RR1-PC & RR1-PC & \\
\hline 80.6953 .1751 & 0.3517135 & RR1-BL2 & RR1-BL2 & RR1-BL2+PC & $\Delta f=0.0280$ \\
\hline 80.6954 .1181 & 0.2863514 & RR1-S & RR1-S & RR1-D & in $r$ \\
\hline 80.6954 .1386 & 0.3015044 & RR1-S & RR1-S & RR1-S & \\
\hline 80.6957 .409 & 0.4078831 & RR1-BL2 & RR1-BL2 & RR1-BL2 & $\Delta f=.0008$, not exactly symm. \\
\hline 80.6958 .1037 & 0.2752632 & RR1-S & RR1-BL2 & RR1-BL2 & $\Delta f=.0343$ \\
\hline 80.6959 .3673 & 0.3311194 & RR1-S & RR1-S & RR1-S & \\
\hline 80.7071 .5289 & 0.3572094 & RR01 & RR01 & RR01 & \\
\hline 80.7072 .1233 & 0.3677101 & RR01 & RR01 & RR01 & \\
\hline 80.7072 .1545 & 0.3583444 & RR1-PC & RR1-PC & RR1-BL2 & $\Delta f= \pm 0.0013$ \\
\hline 80.7072 .2154 & 0.3608532 & RR01 & RR01 & RR01 & \\
\hline 80.7072 .2280 & 0.2786360 & RR1-BL1 & RR1-BL1 & RR1-BL1 & $\Delta f=-0.0472$ \\
\hline 80.7073 .1658 & 0.3478323 & RR01 & RR01 & RR01 & \\
\hline 80.7073 .2115 & 0.3524070 & RR1-S & RR1-S & RR1-S & weak RR1-D in $r$ \\
\hline 80.7074 .1175 & 0.3059442 & RR1-S & RR1-D & RR1-D & in $b+$ peak at 1.2 in $b$ \\
\hline 80.7075 .1977 & 0.3284916 & RR1-S & RR1-S & RR1-D & in $r$ \\
\hline 80.7077 .750 & 0.4043715 & RR1-S & RR1-S & RR1-S & \\
\hline 80.7192 .3592 & 0.4059588 & RR1-PC & RR1-PC & RR1-PC & wide, asymm. $P=0.40599526 ?$ \\
\hline 80.7192 .4319 & 0.3495739 & RR1-S & RR1-S & RR1-D & in $b$ \\
\hline 80.7192 .4927 & 0.3162052 & RR1-S & RR1-S & RR1-S & \\
\hline 80.7193 .1485 & 0.3288181 & RR01 & RR01 & RR01 & $v_{1}=3.04, v_{0}=2.26$ \\
\hline
\end{tabular}


Table 5. continued.

\begin{tabular}{|c|c|c|c|c|c|}
\hline MACHO ID & $\begin{array}{c}\text { Period } \\
\text { [d] }\end{array}$ & $\begin{array}{l}\text { Type } r \\
\text { (A00) }\end{array}$ & $\begin{array}{l}\text { Type } r, b \\
(\text { SAM) } \\
6.5 \text { years }\end{array}$ & $\begin{array}{l}\text { Type } r, b \\
\text { (SAM) } \\
7.5 \text { years }\end{array}$ & 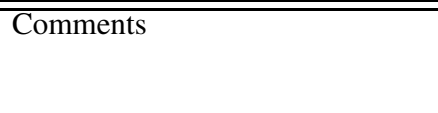 \\
\hline 80.7195 .1166 & 0.3107549 & $\overline{\mathrm{RR} 1-\mathrm{PC}}$ & RR1-PC & RR1-PC & \\
\hline 80.7200 .1403 & 0.2615610 & RR1-S & RR1-S & RR1-S & + rem at $v_{0}$ in $r$ \\
\hline 80.7200 .997 & 0.3092638 & RR1-S & RR1-S & RR1-S & \\
\hline 80.7201 .3035 & 0.3191099 & RR1-S & RR1-S & RR1-S & crossID: 3.7201 .504 \\
\hline 80.7202 .4678 & 0.3571148 & RR01 & RR01 & RR01 & crossID: 3.7202 .916 \\
\hline 80.7313 .3932 & 0.3406024 & RR1-S & RR1-D & RR1-D & $v_{1}=4.008$ \\
\hline 80.7313 .4672 & 0.3681655 & RR01 & RR01 & RR01 & \\
\hline 80.7314 .1485 & 0.3081994 & RR1-S & RR1-S & RR1-S & \\
\hline 80.7315 .1237 & 0.3191161 & RR1-S & RR1-PC & RR1-PC & \\
\hline 80.7315 .1538 & 0.3533676 & RR01 & RR01 & RR01 & \\
\hline 80.7316 .1285 & 0.3655236 & RR01 & RR01 & RR01 & \\
\hline 80.7319 .1287 & 0.2978598 & RR1-PC & RR1-PC & RR1-BL2 & $\Delta f=.0009$ \\
\hline 80.7320 .1224 & 0.3397082 & RR1-S & RR1-S & RR1-S & \\
\hline 80.7321 .1365 & 0.3706183 & RR01 & RR01 & RR01 & \\
\hline 80.7322 .3124 & 0.3180059 & RR1-S & RR1-S & RR1-S & crossID: 3.7322 .614 \\
\hline 80.7434 .4258 & 0.3357009 & RR1-S & RR1-S & RR1-S & \\
\hline 80.7436 .1309 & 0.3692421 & RR1-S & RR1-S & RR1-PC & \\
\hline 80.7436 .1463 & 0.3025085 & RR1-PC & RR1-PC & RR1-PC & \\
\hline 80.7436 .1633 & 0.3304713 & RR1-PC & RR1-PC & RR1-PC & \\
\hline 80.7437 .1665 & 0.3377207 & RR1-S & RR1-S & RR1-S & \\
\hline 80.7437 .1678 & 0.2781401 & RR1-BL1 & RR1-BL1 & RR1-BL1 & $\Delta f=-.0889$, weak RR1-MC too \\
\hline 80.7439 .1826 & 0.3943811 & RR01 & RR01 & RR01 & \\
\hline 80.7439 .1836 & 0.4622122 & RR01 & RR01 & RR01 & $v_{1}>v_{0}$ \\
\hline 80.7440 .1192 & 0.3430250 & RR1-PC & RR1-PC & RR1-BL2 & \\
\hline 80.7441 .933 & 0.2733126 & RR1-BL2 & RR1-BL2 & RR1-BL2 & $\Delta f=.0480$ \\
\hline 80.7441 .960 & 0.3689460 & RR01 & RR01 & RR01 & \\
\hline 80.7444 .3505 & 0.3174408 & RR1-PC & RR1-PC & RR1-PC & \\
\hline 80.7556 .850 & 0.3413911 & RR1-S & RR1-S & RR1-PC & weak \\
\hline 80.7558 .650 & 0.3593405 & RR1-S & RR1-S & RR1-MI & MI in $r$ \\
\hline 80.7563 .516 & 0.3420220 & RR1-S & RR1-PC & RR1-PC & symm., $\Delta f=.00045$ \\
\hline 81.8396 .1664 & 0.3507728 & RR1-S & RR1-S & RR1-S & \\
\hline 81.8398 .799 & 0.3265745 & RR1-S & RR1-S & RR1-S & \\
\hline 81.8400 .901 & 0.2739736 & RR1-S & RR1-BL1 & RR1-BL1 & \\
\hline 81.8401 .496 & 0.2883848 & RR1-S & RR1-S & RR1-S & \\
\hline 81.8515 .2414 & 0.3496134 & RR1-S & RR1-S & RR1-S & +weak RR1-D \\
\hline 81.8515 .3056 & 0.3352154 & RR1-S & RR1-S & RR1-S & \\
\hline 81.8518 .1621 & 0.4106231 & RR1-PC & RR1-PC & RR1-PC & \\
\hline 81.8518 .970 & 0.3085487 & RR1-S & RR1-BL1 & RR1-BL1+PC & both $\mathrm{PC}, \Delta f=-0.0150$ \\
\hline 81.8519 .1395 & 0.3393985 & RR1-D & RR1-D & RR1-MI & peak at 0.8372 only in $r$ \\
\hline 81.8520 .1634 & 0.3132956 & RR1-S & RR1-PC & RR1-PC & \\
\hline 81.8521 .1454 & 0.3094402 & RR1-D & RR1-D & RR1-D & in $r$ \\
\hline 81.8635 .2066 & 0.3388585 & RR1-S & RR1-S & RR1-S & \\
\hline 81.8635 .2229 & 0.3172088 & RR1-S & RR1-MI & RR1-S & strange peak at $v_{0} \cdot 0.6965$ \\
\hline 81.8636 .1973 & 0.3696486 & RR1-S & RR1-S & RR1-S & \\
\hline 81.8638 .2973 & 0.3185128 & RR1-S & RR1-S & RR1-S & \\
\hline 81.8639 .1450 & 0.3352574 & RR01 & RR01 & RR01 & \\
\hline 81.8639 .1749 & 0.3759094 & RR1-BL2 & RR1-PC+BL2 & RR1-PC+BL2 & $\Delta f=.0448, \Delta f=.0005$ \\
\hline 81.8639 .662 & 0.2852523 & RR1-S & RR1-S & RR1-S & \\
\hline 81.8640 .1359 & 0.3250148 & RR1-S & RR1-S & RR1-D & + peak at 2.3 in $r$ \\
\hline 81.8641 .1339 & 0.2969103 & RR1-S & RR1-D & RR1-D & in $b$ \\
\hline 81.8642 .1238 & 0.3674166 & RR1-S & RR1-S & RR1-S & \\
\hline 81.8642 .1384 & 0.3407948 & RR1-S & RR1-S & RR1-S & \\
\hline 81.8643 .1218 & 0.2927151 & BI & RR1-S? & RR1-S & $p$. in $r$ at 5.01 RR1-D? \\
\hline 81.8758 .1447 & 0.4562457 & RR1-BL2 & RR1-BL2 & RR0-BL2 & $\Delta f=.0298,2 v_{0}-\Delta f$ too, lc. \\
\hline 81.8759 .832 & 0.3038356 & RR1-S & RR1-S & RR1-BL1 & $\Delta f=+0.0744$ \\
\hline 81.8760 .1697 & 0.3964948 & RR1-S & RR1-S & RR1-S & \\
\hline 81.8761 .2328 & 0.3909220 & RR1-S & RR0-S & RR0-BL1 & $\Delta f=.0007,2 v_{0}-\Delta f$ too, lc. \\
\hline 81.8762 .1416 & 0.3400559 & RR1-S & RR1-S & RR1-S & \\
\hline 81.8764 .909 & 0.2966042 & RR1-S & RR1-S & RR1-S & \\
\hline 81.8875 .2038 & 0.4057937 & RR1-PC & RR1-PC & RR1-PC & \\
\hline 81.8876 .2820 & 0.3511419 & RR1-S & RR1-S & RR1-S & \\
\hline 81.8879 .1869 & 0.2975649 & RR1-PC & RR1-PC & RR1-BL2 & $\Delta f= \pm 0.0008$ \\
\hline 81.8879 .2137 & 0.3264594 & RR1-S & RR1-D & RR1-S & +weak RR1-D \\
\hline 81.8881 .1370 & 0.4093554 & RR1-S & RR1-S & RR1-S & alias in $b$ \\
\hline 81.8882 .1067 & 0.3491217 & RR01 & RR01 & RR01 & \\
\hline
\end{tabular}


Table 5. continued.

\begin{tabular}{|c|c|c|c|c|c|}
\hline MACHO ID & $\begin{array}{c}\text { Period } \\
\text { [d] }\end{array}$ & $\begin{array}{l}\text { Type } r \\
\text { (A00) }\end{array}$ & $\begin{array}{l}\text { Type } r, b \\
\text { (SAM) } \\
6.5 \text { years }\end{array}$ & $\begin{array}{l}\text { Type } r, b \\
\text { (SAM) } \\
7.5 \text { years }\end{array}$ & 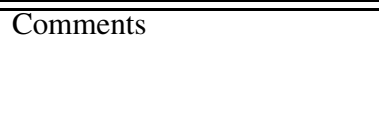 \\
\hline 81.8882 .1422 & 0.3353903 & RR1-S & RR1-S & RR1-S & \\
\hline 81.8884 .1212 & 0.3703148 & RR1-S & RR1-S & RR1-S & \\
\hline 81.8885 .1203 & 0.2817928 & RR1-S & RR1-S & RR1-S & weak RR1-D in $r,+$ stuff in $b$ \\
\hline 81.9001 .2673 & 0.3558753 & RR1-S & RR1-S & RR1-S & \\
\hline 81.9004 .1177 & 0.3180302 & RR1-PC & RR1-PC & RR1-PC & \\
\hline 81.9117 .1184 & 0.3295384 & RR1-S & RR1-D & RR1-D & \\
\hline 81.9118 .1794 & 0.3501430 & RR01 & RR01 & RR01 & \\
\hline 81.9120 .1324 & 0.3350516 & RR1-S & RR1-S & RR1-S & \\
\hline 81.9121 .1477 & 0.3001045 & RR1-S & RR1-S & RR1-S & \\
\hline 81.9123 .806 & 0.3853143 & RR1-PC & RR1-PC & RR1-PC & \\
\hline 81.9247 .1035 & 0.2910902 & RR1-S & RR1-S & RR1-S & \\
\hline 81.9247 .1164 & 0.3268370 & RR1-S & RR1-S & RR1-S & \\
\hline 81.9247 .1293 & 0.3928922 & RR1-S & RR1-S & RR1-S & \\
\hline 81.9248 .1307 & 0.3235209 & RR1-S & RR1-S & RR1-S & \\
\hline 81.9366 .1335 & 0.3098233 & RR1-S & RR1-S & RR1-D & in $b$ \\
\hline 81.9368 .944 & 0.3381468 & RR1-S & RR1-S & RR1-S & \\
\hline 81.9481 .624 & 0.3967379 & BI & BI & BI & light curve \\
\hline 81.9482 .1608 & 0.3502312 & RR1-S & RR1-S & RR1-S & \\
\hline 81.9484 .1382 & 0.2713864 & RR1-S & RR1-S & RR1-S & \\
\hline 81.9486 .734 & 0.3008852 & RR1-S & RR1-PC & RR1-PC & alias \\
\hline 81.9601 .1319 & 0.3241070 & RR1-S & RR1-S & RR1-S & peak in $r$ at $\approx 3.85$ \\
\hline 81.9603 .1133 & 0.3397803 & RR1-S & RR1-S & RR1-S & \\
\hline 81.9604 .1054 & 0.2801471 & RR1-S & RR1-S & RR1-S & \\
\hline 81.9611 .399 & 0.4005153 & RR1-S & RR1-PC & RR1-PC & \\
\hline 81.9723 .796 & 0.3369516 & RR1-PC & RR1-PC & RR1-PC & wide \\
\hline 81.9723 .894 & 0.3886024 & RR1-PC & RR1-PC & RR1-PC & + very weak RR1-BL1 \\
\hline 81.9724 .295 & 0.3538641 & RR1-PC & RR1-PC & RR1-PC & wide \\
\hline 82.7920 .1074 & 0.3386711 & RR1-S & RR1-S & RR1-BL1 & weak \\
\hline 82.7921 .759 & 0.3564993 & RR1-S & RR1-S & RR1-D & mostly in $b$ \\
\hline 82.7921 .822 & 0.3497370 & RR1-S & RR1-S & RR1-S & \\
\hline 82.7921 .827 & 0.3281433 & RR1-S & RR1-S & RR1-S & \\
\hline 82.7922 .520 & 0.3635551 & RR01 & RR01 & RR01 & \\
\hline 82.7922 .838 & 0.3253781 & RR1-S & RR1-S & RR1-S & \\
\hline 82.7924 .1100 & 0.3090396 & RR1-S & RR1-S & RR1-S & weak p. in $r$ at $\approx 2 v_{0}-1$ \\
\hline 82.7928 .580 & 0.3521369 & RR1-S & RR1-S & RR1-D & \\
\hline 82.8040 .1189 & 0.2979495 & RR1-S & RR1-D & RR1-D & in $b$ \\
\hline 82.8041 .1029 & 0.4067153 & RR1-PC & RR1-PC & RR1-PC & \\
\hline 82.8042 .1304 & 0.2885570 & RR1-S & RR1-S & RR1-S & \\
\hline 82.8043 .1209 & 0.3339364 & RR1-S & RR1-S & RR1-PC & \\
\hline 82.8043 .1438 & 0.3055072 & RR1-S & RR1-S & RR1-S & \\
\hline 82.8046 .1235 & 0.3144617 & RR1-S & RR1-S & RR1-S & weak RR1-D \\
\hline 82.8047 .999 & 0.3409796 & RR1-S & RR1-S & RR1-S & \\
\hline 82.8048 .806 & 0.3462522 & RR1-S & RR1-D & RR1-D & peak in $b$ at $\approx 2 v_{0}$ \\
\hline 82.8049 .746 & 0.2987354 & RR1-BL2 & RR1-BL2 & RR1-BL2 & $\Delta f=.0720,2 v_{0}-\Delta f$ too \\
\hline 82.8049 .898 & 0.3662099 & RR01 & RR01 & RR01 & \\
\hline 82.8160 .3605 & 0.2756421 & RR1-S & RR1-S & RR1-S & $\mathrm{P}(\mathrm{A} 00) \neq \mathrm{P}(\mathrm{SAM})$ \\
\hline 82.8161 .1231 & 0.4045666 & RR1-S & RR1-PC & RR1-PC & strong aliasing \\
\hline 82.8163 .1039 & 0.3677745 & RR01 & RR01 & RR01 & \\
\hline 82.8163 .1275 & 0.2637632 & RR1-S & RR1-S & RR1-S & \\
\hline 82.8164 .932 & 0.2781573 & RR1-S & RR1-S & RR1-S & \\
\hline 82.8167 .864 & 0.3933491 & RR1-S & RR1-BL1? & RR1-PC & around $2 v_{0}$ too \\
\hline 82.8168 .1006 & 0.3531867 & RR1-S & RR1-S & RR1-S & \\
\hline 82.8168 .1014 & 0.3709171 & RR1-S & RR1-S & RR1-S & \\
\hline 82.8168 .1144 & 0.3335168 & RR1-S & RR1-S & RR1-D & weak, $\mathrm{P}(\mathrm{A} 00) \neq \mathrm{P}(\mathrm{SAM})$ \\
\hline 82.8169 .1038 & 0.2954577 & RR1-S & RR1-S & RR1-S & \\
\hline 82.8169 .654 & 0.3539979 & RR01 & RR01 & RR01 & \\
\hline 82.8282 .1019 & 0.3271100 & RR1-PC & RR1-PC & RR1-PC & \\
\hline 82.8283 .1040 & 0.3700767 & RR1-PC & RR1-PC & RR1-PC & \\
\hline 82.8284 .1105 & 0.3421157 & RR1-S & RR1-S & RR1-S & \\
\hline 82.8285 .733 & 0.3505385 & RR1-S & RR1-S & RR1-S & \\
\hline 82.8285 .967 & 0.3923695 & RR1-S & RR1-S & RR1-S & \\
\hline 82.8286 .1733 & 0.3403737 & RR1-S & RR1-S & RR1-S & \\
\hline 82.8286 .1784 & 0.3285773 & RR1-S & RR1-PC & RR1-BL1 & one peak, whitens out \\
\hline 82.8288 .869 & 0.3740560 & RR1-PC & RR1-PC & RR1-PC & \\
\hline 82.8289 .887 & 0.2829623 & RR1-BL1 & RR1-BL1 & RR1-MC & $\Delta v_{1}=-.1896 \Delta v_{2}=\Delta v_{1} / 2$ \\
\hline
\end{tabular}


Table 5. continued.

\begin{tabular}{|c|c|c|c|c|c|}
\hline MACHO ID & $\begin{array}{c}\text { Period } \\
\text { [d] }\end{array}$ & $\begin{array}{l}\text { Type } r \\
\text { (A00) }\end{array}$ & $\begin{array}{l}\text { Type } r, b \\
\text { (SAM) } \\
6.5 \text { years }\end{array}$ & $\begin{array}{l}\text { Type } r, b \\
\text { (SAM) } \\
7.5 \text { years }\end{array}$ & 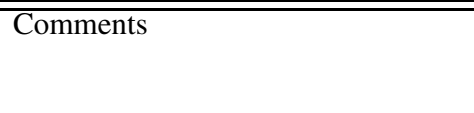 \\
\hline 82.8289 .894 & 0.2765946 & RR1-S & RR1-S? & RR1-S & \\
\hline 82.8289 .975 & 0.3191820 & RR1-S & RR1-S & RR1-S & \\
\hline 82.8291 .748 & 0.3453027 & RR1-S & RR1-S & RR1-S & \\
\hline 82.8403 .1229 & 0.3638096 & RR1-S & RR1-S & RR1-D & \\
\hline 82.8403 .1251 & 0.2821401 & RR1-S & RR1-D & RR1-D & mostly in $r$ \\
\hline 82.8406 .1180 & 0.3738827 & RR1-PC & RR1-PC & RR1-PC & \\
\hline 82.8407 .1628 & 0.3761618 & RR1-S & RR1-S & RR1-S & \\
\hline 82.8407 .312 & 0.3563439 & RR1-S & RR1-D & RR1-BL1 & $\Delta f=.0008$ \\
\hline 82.8408 .1002 & 0.2962930 & RR1-BL2 & RR1-BL2 & RR1-BL2 & $\Delta f=.0354$ \\
\hline 82.8410 .986 & 0.3483861 & RR01 & RR01 & RR01 & \\
\hline 82.8411 .841 & 0.3394055 & RR1-S & RR1-S & RR1-S & \\
\hline 82.8525 .1778 & 0.3518382 & RR1-S & RR1-S & RR1-S & \\
\hline 82.8525 .1980 & 0.2833982 & RR1-MI & RR1-S & RR1-BL1 & $\Delta f=0.0841$ \\
\hline 82.8526 .1176 & 0.3238862 & RR1-BL2 & RR1-BL2 & RR1-BL2 & $\Delta f=0.1270$ \\
\hline 82.8527 .1433 & 0.3384423 & RR1-S & RR1-S & RR1-S & \\
\hline 82.8530 .1009 & 0.4020850 & RR1-S & RR1-S & RR1-PC & in $r$ \\
\hline 82.8533 .1307 & 0.2806150 & RR1-S & RR1-D & RR1-D & \\
\hline 82.8646 .1074 & 0.3456339 & RR1-S & RR1-S & RR1-S & \\
\hline 82.8646 .1162 & 0.3446226 & RR1-S & RR1-S & RR1-S & \\
\hline 82.8652 .908 & 0.3729295 & RR1-S & RR1-S & RR1-S & \\
\hline 82.8765 .1250 & 0.3049238 & RR1-BL2 & RR1-BL2 & RR1-BL2 & $\Delta f=0.0490,+$ weak RR1-PC \\
\hline 82.8766 .1305 & 0.2592516 & RR1-BL1 & RR1-BL1 & RR1-BL1 & $\Delta f=.0031$ \\
\hline 82.8886 .1146 & 0.3570008 & RR01 & RR01 & RR01 & \\
\hline 82.8889 .452 & 0.3601860 & RR01 & RR01 & RR01 & \\
\hline 82.8890 .449 & 0.4400493 & RR1-PC & RR1-PC & RR1-PC & aliases \\
\hline 82.8894 .808 & 0.3412773 & RR1-S & RR1-S & RR1-S & \\
\hline 82.9015 .1092 & 0.3013519 & RR1-S & RR1-S & RR1-S & \\
\hline 82.9016 .731 & 0.3041599 & RR1-S & RR1-S & RR1-S & \\
\hline 82.9017 .426 & 0.3222430 & RR1-S & RR1-PC & RR1-PC & \\
\hline 82.9130 .408 & 0.3490416 & RR1-S & RR01? & RR1-S & \\
\hline 82.9138 .552 & 0.3516634 & RR1-S & RR1-PC & RR1-S & \\
\hline 9.4270 .541 & 0.3193464 & RR1-S & RR1-S & RR1-S & \\
\hline 9.4270 .685 & 0.2773749 & RR1-S & RR1-S & RR1-S & \\
\hline 9.4273 .1378 & 0.3606228 & RR1-S & RR1-S & RR1-S & \\
\hline 9.4274 .644 & 0.2636423 & RR1-S & RR1-S & RR1-BL1 & $\Delta f= \pm .1230$, " + " is weak, in $b$ \\
\hline 9.4275 .536 & 0.3530716 & RR01 & RR01 & RR01 & \\
\hline 9.4276 .488 & 0.3301639 & RR1-S & RR1-S & RR1-S & crossID: 10.4276 .3455 \\
\hline 9.4278 .179 & 0.3268162 & MDM & MDM & MDM & V18.92 R18.01 \\
\hline 9.4390 .560 & 0.4110355 & RR1-PC & RR1-PC & RR1-PC & \\
\hline 9.4392 .625 & 0.3445635 & RR1-S & RR1-PC & RR1-PC & \\
\hline 9.4392 .833 & 0.3034402 & RR1-PC & RR1-PC & RR1-PC & \\
\hline 9.4393 .761 & 0.2755079 & RR1-S & RR1-S & RR1-S & \\
\hline 9.4394 .386 & 0.2907779 & RR1-PC & RR1-PC & RR1-PC & aliases \\
\hline 9.4398 .729 & 0.3048604 & RR1-S & RR1-S & RR1-S & crossID: 10.4398 .4446 \\
\hline 9.4398 .852 & 0.3325725 & RR1-S & RR1-S & RR1-S & crossID: 10.4398 .4412 \\
\hline 9.4512 .701 & 0.3446228 & RR1-S & RR1-S & RR1-S & weak p. in $b$ at $\approx .9$ \\
\hline 9.4513 .866 & 0.2852547 & RR1-S & RR1-S & RR1-S & \\
\hline 9.4514 .979 & 0.3574786 & RR01 & RR01 & RR01 & \\
\hline 9.4516 .965 & 0.3125994 & RR1-S & RR1-S & RR1-D & double peak \\
\hline 9.4516 .980 & 0.2741678 & RR1-S & RR1-D & RR1-D & in $b$ \\
\hline 9.4520 .778 & 0.2852016 & RR1-S & RR1-D & RR1-D & in $b$, crossID: 10.4520 .4862 \\
\hline 9.4520 .952 & 0.3365915 & RR1-S & RR1-S & RR1-S & + stuff in $b$, crossID: 10.4520 .5022 \\
\hline 9.4632 .592 & 0.2802225 & RR1-S & RR1-S & RR1-S & +weak RR1-D in $r$ \\
\hline 9.4632 .731 & 0.2775501 & RR1-S & RR1-BL2 & RR1-BL2 & $\Delta f=0.00668$ \\
\hline 9.4632 .793 & 0.3406884 & RR1-S & RR1-S & RR1-S & + weak peak in $r$ at $\approx 2.2$ \\
\hline 9.4634 .863 & 0.3981110 & RR1-PC & RR1-PC & RR1-PC & +very weak RR1-BL1, 6.21, +.0963 \\
\hline 9.4635 .790 & 0.3607762 & RR1-S & RR1-S & RR1-D & in $b$ \\
\hline 9.4635 .878 & 0.3463833 & RR01 & RR01 & RR01 & \\
\hline 9.4636 .1979 & 0.3933065 & RR1-S & RR1-PC? & RR1-BL1 & $\Delta f=.0005$, whitens out \\
\hline 9.4638 .870 & 0.3307464 & RR1-S & RR1-S & RR1-S & + str. in b at $v_{0} / v_{1}=.7013$ \\
\hline 9.4639 .933 & 0.3314458 & RR1-S & RR1-S & RR1-S & + weak RR1-D in $b$ \\
\hline 9.4640 .1003 & 0.2995329 & RR1-S & RR1-S & RR1-S & + weak rem. at $v_{0}$ \\
\hline 9.4753 .921 & 0.3704734 & BI & BI & BI & $v_{1} / v_{0}$, light curve \\
\hline 9.4755 .739 & 0.3368453 & RR1-S & RR1-S & RR1-S & \\
\hline 9.4758 .925 & 0.3173162 & RR1-S & RR1-S & RR1-S & \\
\hline
\end{tabular}


Table 5. continued.

\begin{tabular}{|c|c|c|c|c|c|}
\hline MACHO ID & $\begin{array}{c}\text { Period } \\
\text { [d] }\end{array}$ & $\begin{array}{l}\text { Type } r \\
\text { (A00) }\end{array}$ & $\begin{array}{c}\text { Type } r, b \\
\text { (SAM) } \\
6.5 \text { years }\end{array}$ & $\begin{array}{l}\text { Type } r, b \\
\text { (SAM) } \\
7.5 \text { years }\end{array}$ & Comments \\
\hline 9.4759 .891 & 0.3694758 & RR1-S & RR1-D & RR1-S & weak RR1-D in $b$ \\
\hline 9.4760 .1004 & 0.3102123 & RR1-S & RR1-D & RR1-D & in $b$ \\
\hline 9.4760 .861 & 0.2996671 & RR1-PC & RR1-PC & RR1-PC & \\
\hline 9.4760 .945 & 0.3248036 & RR1-S & RR1-S & RR1-S & \\
\hline 9.4761 .1258 & 0.3009386 & RR1-PC & RR1-PC & RR1-PC & \\
\hline 9.4761 .966 & 0.3395877 & RR1-S & RR1-S & RR1-S & weak RR1-D \\
\hline 9.4762 .1015 & 0.3454185 & RR1-S & RR1-S & RR1-S & \\
\hline 9.4762 .1072 & 0.3105078 & RR1-S & RR1-S & RR1-S & \\
\hline 9.4873 .497 & 0.3586208 & RR1-PC & RR1-PC & RR1-PC & \\
\hline 9.4873 .519 & 0.3512584 & RR01 & RR01 & RR01 & \\
\hline 9.4875 .697 & 0.3206464 & RR1-PC & RR1-PC & RR1-PC & \\
\hline 9.4875 .852 & 0.3130636 & RR1-S & RR1-S & RR1-D & in $b$ \\
\hline 9.4877 .802 & 0.3420082 & RR1-S & RR1-S & RR1-S & \\
\hline 9.4878 .2126 & 0.3375846 & RR1-S & RR1-BL1 & RR1-PC & \\
\hline 9.4879 .502 & 0.3982046 & RR1-PC & RR1-PC & RR1-PC & \\
\hline 9.4879 .550 & 0.3791640 & RR1-S & RR1-S & RR1-PC & \\
\hline 9.4880 .858 & 0.3537984 & RR1-S & RR1-S & RR1-D & \\
\hline 9.4881 .635 & 0.3663045 & RR01 & RR01 & RR01 & \\
\hline 9.4882 .1059 & 0.2763814 & RR1-S & RR1-S & RR1-S & \\
\hline 9.4883 .1241 & 0.3602522 & RR1-S & RR1-S & RR1-S & \\
\hline 9.4994 .491 & 0.3664183 & RR1-PC & RR1-PC & RR1-PC & \\
\hline 9.4995 .551 & 0.3266079 & RR1-S & RR1-S & RR1-S & \\
\hline 9.4998 .709 & 0.3942098 & BI & BI & BI & $v_{1} / v_{0}$, light curve \\
\hline 9.4998 .725 & 0.2971509 & RR1-S & RR1-MI & RR1-D & peak at .1163 in $b$ only \\
\hline 9.4999 .1530 & 0.2869667 & RR1-S & RR1-S & RR1-D & in $b$ \\
\hline 9.4999 .1762 & 0.3194617 & RR1-S & RR1-MI? & $\mathrm{BI}$ & $v_{1} / v_{0}$, light curve rather eclipse \\
\hline 9.5001 .708 & 0.3383914 & RR1-S & RR1-S & RR1-S & \\
\hline 9.5001 .841 & 0.3400434 & RR1-S & RR1-S & RR1-S & \\
\hline 9.5002 .709 & 0.3014034 & RR1-S & RR1-S & RR1-S & \\
\hline 9.5003 .684 & 0.2862712 & RR1-S & RR1-S & RR1-S & weak RR1-D in $b$ \\
\hline 9.5003 .978 & 0.3597825 & RR1-S & RR1-S & RR1-S & \\
\hline 9.5004 .750 & 0.3041610 & RR1-D & RR1-D & RR1-MI & $v_{2}=.1605$ \\
\hline 9.5115 .785 & 0.3485641 & BI & BI & BI & $v_{1} / v_{0}$, light curve \\
\hline 9.5117 .617 & 0.3601338 & RR1-S & RR1-PC & RR1-BL2 & symm, $\Delta f=.0005$, whitens out \\
\hline 9.5117 .696 & 0.3466028 & RR1-S & RR1-S & RR1-S & + rem at $v_{0}$ \\
\hline 9.5117 .814 & 0.2760081 & RR1-S & RR1-S & RR1-S & \\
\hline 9.5119 .644 & 0.3565925 & RR01 & RR01 & RR01 & \\
\hline 9.5121 .767 & 0.3530495 & RR1-S & RR1-S & RR1-S & \\
\hline 9.5121 .827 & 0.3518164 & RR01 & RR01 & RR01 & \\
\hline 9.5122 .363 & 0.3222320 & RR1-D & RR1-D & RR1-MI & $v_{0} / v_{2}=.7446$ \\
\hline 9.5123 .1127 & 0.2982710 & RR1-S & RR1-S & RR1-S & \\
\hline 9.5123 .284 & 0.2995174 & RR1-S & RR1-S & RR1-S & \\
\hline 9.5123 .633 & 0.2677299 & RR1-MI & RR1-MI & RR01 & \\
\hline 9.5123 .713 & 0.3702883 & RR1-S & RR1-S & RR1-S & \\
\hline 9.5124 .1174 & 0.3535816 & RR01 & RR01 & RR01 & \\
\hline 9.5124 .850 & 0.3051548 & RR1-S & RR1-S & RR1-S & \\
\hline 9.5125 .1018 & 0.2598093 & RR1-BL2 & RR1-BL2 & RR1-BL2 & $\Delta f=.0052$, in $r$ \\
\hline 9.5236 .587 & 0.3616035 & RR1-S & RR1-S & RR1-S & \\
\hline 9.5236 .675 & 0.3430403 & RR1-S & RR1-D & RR1-S & \\
\hline 9.5238 .724 & 0.2948677 & RR1-S & RR1-S & RR1-S & \\
\hline 9.5238 .784 & 0.3311550 & RR1-S & RR1-S & RR1-S & \\
\hline 9.5238 .804 & 0.3655498 & RR01 & RR01 & RR01 & \\
\hline 9.5239 .1141 & 0.3633337 & $\mathrm{NC}$ & RR1-PC & RR1-PC & $(\mathrm{RRc}) \mathrm{P}(\mathrm{A} 00) \neq \mathrm{P}(\mathrm{SAM}) \mathrm{RR} 1-\mathrm{D}$ in $r$ \\
\hline 9.5239 .728 & 0.3530979 & RR1-S & RR1-S & RR1-S & \\
\hline 9.5240 .750 & 0.3187437 & RR1-S & RR1-S & RR1-S & \\
\hline 9.5241 .1725 & 0.2911409 & RR1-S & RR1-S & RR1-S & \\
\hline 9.5241 .1868 & 0.3594974 & RR1-S & RR1-S & RR1-S & +weak RR1-D in $r$ \\
\hline 9.5241 .382 & 0.3416840 & RR1-S & RR1-S & RR1-MI & peak at 2.35 in $b$ \\
\hline 9.5242 .1032 & 0.2858950 & RR1-BL1 & RR1-BL1 & RR1-BL1 & $\Delta f=+0.0375$ \\
\hline 9.5242 .974 & 0.3194565 & RR1-S & RR1-S & RR1-S & \\
\hline 9.5243 .1131 & 0.3293029 & RR1-S & RR1-S & RR1-S & \\
\hline 9.5243 .955 & 0.3595260 & RR1-S & RR1-S & RR1-S & \\
\hline 9.5245 .1046 & 0.3217844 & RR1-S & RR1-D & RR1-D & in $r$ \\
\hline 9.5245 .1190 & 0.3506518 & RR1-S & RR1-D & RR1-D & in $r$ \\
\hline 9.5246 .989 & 0.3135361 & RR1-S & RR1-D & RR1-D & \\
\hline
\end{tabular}


Table 5. continued.

\begin{tabular}{|c|c|c|c|c|c|}
\hline$\overline{\mathrm{MACHO} \text { ID }}$ & $\begin{array}{c}\overline{\text { Period }} \\
\text { [d] }\end{array}$ & $\begin{array}{l}\text { Type } r \\
\text { (A00) }\end{array}$ & $\begin{array}{l}\text { Type } r, b \\
\text { (SAM) } \\
6.5 \text { years }\end{array}$ & $\begin{array}{l}\text { Type } r, b \\
\text { (SAM) } \\
7.5 \text { years }\end{array}$ & $\overline{\text { Comments }}$ \\
\hline 9.5358 .566 & 0.3710141 & RR01 & RR01 & RR01 & \\
\hline 9.5359 .671 & 0.2893691 & RR1-S & RR1-D & RR1-D & in $b$ \\
\hline 9.5359 .714 & 0.3537696 & RR1-S & RR1-S & RR1-S & \\
\hline 9.5360 .768 & 0.3375177 & RR1-D & RR1-D & RR1-D & \\
\hline 9.5360 .773 & 0.3615600 & RR1-S & RR1-D & RR1-D & \\
\hline 9.5360 .782 & 0.3075059 & RR1-S & RR1-S & RR1-S & \\
\hline 9.5360 .853 & 0.3390032 & RR1-S & RR1-S & RR1-S & peak in $r$ at detection limit \\
\hline 9.5360 .903 & 0.4138971 & RR1-PC & RR1-PC & RR1-PC & + weak RR1-D \\
\hline 9.5361 .694 & 0.3125844 & RR1-S & RR1-S & RR1-D & in $r$ \\
\hline 9.5361 .900 & 0.3576700 & RR1-S & RR1-S & RR1-S & rem. at $v_{0}+1 ; \mathrm{P}(\mathrm{A} 00) \neq \mathrm{P}(\mathrm{SAM})$ \\
\hline 9.5362 .1886 & 0.3423878 & RR1-S & RR1-S & RR1-S & \\
\hline 9.5362 .314 & 0.3975989 & RR1-S & RR1-S & RR1-S & \\
\hline 9.5362 .324 & 0.3447272 & RR1-S & RR1-S & RR1-S & \\
\hline 9.5363 .899 & 0.3640478 & RR1-PC & RR1-PC & RR1-PC & \\
\hline 9.5364 .886 & 0.3987656 & RR1-PC & RR1-PC & RR1-MC & \\
\hline 9.5365 .878 & 0.3149004 & RR1-S & RR1-D & RR1-D & \\
\hline 9.5365 .907 & 0.3172517 & RR1-S & RR1-S & RR1-S & \\
\hline 9.5367 .1206 & 0.3226902 & RR1-S & RR1-S & BI & $v_{1} / v_{0}$, light curve rather eclipse \\
\hline 9.5367 .959 & 0.3399758 & RR1-S & RR1-S & RR1-S & \\
\hline 9.5478 .719 & 0.3074701 & RR1-S & RR1-PC & RR1-PC & whitens out \\
\hline 9.5478 .786 & 0.2833626 & RR1-S & RR1-S & RR1-S & \\
\hline 9.5479 .1190 & 0.2793167 & RR1-S & RR1-BL1 & RR1-S & $\mathrm{S} / \mathrm{N}$ was 6.67 , it is 5.92 now...!!! \\
\hline 9.5479 .751 & 0.3464845 & RR1-S & RR1-S? & RR1-S & + peak at $1.1971 \mathrm{in} r$ \\
\hline 9.5479 .852 & 0.3217303 & RR1-BL2 & RR1-BL2 & RR1-BL2 & $\Delta f= \pm .0008$, rem \\
\hline 9.5481 .746 & 0.3581725 & RR1-S & RR1-PC & RR1-BL2 & $\Delta f= \pm .0005$, wh. Aliases! \\
\hline 9.5481 .871 & 0.3513143 & RR1-S & RR1-S & RR1-S & weak rem. at $v_{0} ; \mathrm{P}(\mathrm{A} 00) \neq \mathrm{P}(\mathrm{SAM})$ \\
\hline 9.5481 .965 & 0.3244519 & RR1-S & RR1-D? & RR1-D & in $r$ \\
\hline 9.5482 .1335 & 0.3813366 & RR1-S & RR1-S & RR1-S & \\
\hline 9.5482 .824 & 0.2996470 & RR1-S & RR1-S & RR1-PC & \\
\hline 9.5482 .883 & 0.2724089 & RR1-S & RR1-S? & RR1-S & weak stuff \\
\hline 9.5482 .933 & 0.3339844 & RR1-S & RR1-D? & RR1-S & \\
\hline 9.5482 .957 & 0.3405742 & RR01 & RR01 & RR01 & \\
\hline 9.5483 .1857 & 0.3672216 & RR01 & RR01 & RR01 & \\
\hline 9.5483 .1931 & 0.3409496 & RR1-S & RR1-S & RR1-S & \\
\hline 9.5484 .705 & 0.3316851 & RR1-S & RR1-S & RR1-S & \\
\hline 9.5484 .829 & 0.3577133 & RR1-S & RR1-S & RR1-D & \\
\hline 9.5486 .777 & 0.2982475 & RR1-S & RR1-S & RR1-S & \\
\hline 9.5487 .1048 & 0.3615973 & RR1-S & RR1-MI? & RR1-S & \\
\hline 9.5487 .584 & 0.3692732 & RR1-S & RR1-PC & RR1-PC & alias \\
\hline 9.5487 .736 & 0.3506117 & RR01 & RR01 & RR01 & \\
\hline 9.5599 .617 & 0.2782061 & RR1-D & RR1-D & RR1-D & \\
\hline 9.5599 .682 & 0.4102560 & BI & BI & BI & $v_{1} / v_{0}$, light curve sin. \\
\hline 9.5599 .762 & 0.2994160 & RR1-PC & RR1-PC & RR1-PC & \\
\hline 9.5600 .566 & 0.3313306 & RR1-PC & RR1-PC & RR1-PC & rem. at $2 v_{0}$ too \\
\hline 9.5600 .609 & 0.3046859 & RR1-S & RR1-S & RR1-S & \\
\hline 9.5602 .813 & 0.3163610 & RR1-S & RR1-S & RR1-S & \\
\hline 9.5602 .987 & 0.2975537 & RR1-S & RR1-S & RR1-S & \\
\hline 9.5606 .348 & 0.2909644 & RR1-S & RR1-BL2 & RR1-BL2 & $\Delta f=.0712$ \\
\hline 9.5606 .829 & 0.3563769 & RR1-S & RR1-S & RR1-S & \\
\hline 9.5606 .838 & 0.3530134 & RR1-S & RR1-S & RR1-S & \\
\hline 9.5606 .882 & 0.2647507 & RR1-S & RR1-S & RR1-S & \\
\hline 9.5606 .884 & 0.3096000 & RR1-S & RR1-S & RR1-S & \\
\hline 9.5609 .856 & 0.2848128 & RR1-S & RR1-S & RR1-D & stuff in $b$ at 1.6329 \\
\hline 9.5609 .956 & 0.2707240 & RR1-S & RR1-S & RR1-D & in $b$ \\
\hline
\end{tabular}

Notes: Additional data (positions, magnitudes, etc.) can be found at the MACHO on-line database (http://wwwmacho.mcmaster.ca/ Data/MachoData.html). Comments are intended to draw attention to the given variable rather than supply detailed description of the peculiarities found. 
Table 7. Data on the first overtone Blazhko stars.

\begin{tabular}{|c|c|c|c|c|c|c|c|c|c|c|c|}
\hline MACHO ID. & $P_{1}$ & $f_{\mathrm{BL}}$ & $A_{+}$ & $A_{0}$ & $A_{-}$ & MACHO ID. & $P_{1}$ & $f_{\mathrm{BL}}$ & $A_{+}$ & $A_{0}$ & $A_{-}$ \\
\hline 10.3552 .745 & 2922940 & 0.037408 & 0.0666 & 0.1280 & $\overline{-}$ & 6.5729 .958 & 0.2778179 & 0.074254 & 0.1045 & 0.1641 & - \\
\hline 57.1024 & 668 & & 000 & 2643 & 09 & 057 & 763214 & 98 & .0662 & 49 & - \\
\hline 095 & 217 & 80 & 96 & 239 & - & 81 & 5893 & -011 & - & 0.1617 & 03 \\
\hline 053 & 579 & 288 & 0.0769 & 1443 & - & 33 & 377179 & 476 & 0.0380 & 868 & $i^{2}$ \\
\hline 1.780 & 825 & 512 & - & 2076 & .0764 & & 8322 & 5389 & - & 1654 & 307 \\
\hline 13.590 & 2836353 & 0.100165 & 0.0636 & .0870 & - & 377 & 206106 & -0.038525 & - & 0.1046 & 642 \\
\hline 13.5714 .442 & 3168726 & 0.109252 & 0.0547 & 1008 & - & 606 & 0.3393854 & -0.001244 & 0.0593 & 0.2476 & .0637 \\
\hline 842.2468 & 0.2731424 & -0.084060 & - & 0.2060 & 0.0355 & 6326.424 & 0.3304519 & -0.072633 & - & 0.2353 & .0504 \\
\hline 13.5959 .584 & .3466458 & 0.000993 & 0.0286 & 2629 & - & 16 & 0.2883850 & -0.000403 & .0552 & 0.2113 & 0.0704 \\
\hline 13.6322 .342 & 2621495 & 0.180083 & 0.0385 & 1166 & - & 13 & 404073 & -0.001674 & 0.0445 & 0.2110 & .0478 \\
\hline 13.6326 .2765 & 3304520 & -0.072702 & - & 2387 & 03. & 36 & 5160 & 0.001120 & 0.0317 & 0.1781 & .0252 \\
\hline 13.6810 .2981 & 2883863 & 0.000419 & 0.06 & 2050 & 0659 & 14 & 0.3079889 & -0.071798 & - & 0.0937 & 682 \\
\hline 13.68 & 3975 & 503 & 0.0909 & 2153 & 8 & 2000 & 5713 & 564 & & 2016 & 472 \\
\hline 14.8 & 105 & & 0.0696 & 36 & 4 & 1599 & 05 & 44 & 0326 & 977 & 812 \\
\hline 14. & 39 & & 0.0941 & 70 & 0 & 35 & & -0 . & 68 & 03 & 19 \\
\hline 14. & 38 & 55 & - & 39 & .090 & 34 & 90 & & 07 & & \\
\hline 14 & 51 & 45 & 0470 & 93 & - & 95 & 97 & 33 & 87 & 21 & 97 \\
\hline 14 & 43 & 06 & - & 49 & 469 & 51 & 35 & -0 . & 77 & 99 & 09 \\
\hline 1. & 49 & 19 & 0325 & 69 & 98 & 409 & 31 & 93 & 37 & 70 & 52 \\
\hline 18 & 7774 & 38 & - & 2971 & 49 & 37 & 32 & -0 & 55 & 41 & 92 \\
\hline .782 & 2903 & 036 & 0.0440 & 2308 & 50 & 45 & 44 & -0.0 & 0.0268 & 236 & 0.0263 \\
\hline .606 & 0.2925053 & 385 & 0.0806 & 1861 & 0.0597 & 2280 & 0.2786360 & -0.0 & - & 0.1486 & 0359 \\
\hline 255 & 2869357 & 243 & 0.1029 & 1030 & - & 287 & 598 & 852 & .0973 & 0.2210 & .0761 \\
\hline 15 & 770 & 580 & - & 2853 & .039 & 678 & & -0 . & 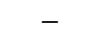 & & 289 \\
\hline & 790 & & 0.0661 & 12 & & 192 & & -0 & .0582 & & .0663 \\
\hline & & & 0.0945 & 690 & & & & & .0551 & 84 & .0420 \\
\hline & & & & & & & & & .0404 & 51 & - \\
\hline & & 51 & 0.0617 & 49 & 20 & & & 72 & - & 192 & .0264 \\
\hline & & & - & & & 49 & & & 59 & 01 & 0363 \\
\hline & & & 6611 & & & & & & & & - \\
\hline & & 84 & 8 & 51 & 0.033 & & & -0 & 14 & 371 & .0660 \\
\hline & & & 0.0705 & & - & 74 & & & 0.0470 & 52 & - \\
\hline & 510 & 69 & 0.0658 & 1821 & 0541 & 46 & 54 & 57 & .0675 & 0.2256 & 0.0482 \\
\hline & 354 & 0.072205 & - & 0.2184 & 47 & 1784 & 773 & -0.000494 & - & 0.1893 & 0.0357 \\
\hline 3. & 6371 & -0.001203 & 0.0641 & 0.2129 & 3 & 312 & 439 & 0.000804 & 0.0371 & 0.2317 & - \\
\hline & 451 & -0.000950 & 0.0304 & 1378 & 7 & 002 & 30 & 08 & 0.0694 & 0.2299 & .0503 \\
\hline 3.6 & 773 & 362 & - & 2527 & 9 & 30 & 82 & 35 & 55 & 0.2378 & - \\
\hline 3.6 & 389 & 466 & 0.064 & 3078 & 0.056 & 76 & 362 & 004 & 0.0321 & 0.1293 & 0312 \\
\hline 3.6 & & & 0.0272 & 48 & - & & 38 & -0 & .0422 & 0.2267 & .0538 \\
\hline & 180 & -0.000642 & 0.0530 & 0.2485 & 0.0676 & 1305 & 0.2592516 & -0.0 & - & 0.0936 & 0.0518 \\
\hline 47. & 36 & 303 & 0.0575 & 0.2303 & 0.0634 & & 423 & -0.122986 & - & 0.1529 & 0.0343 \\
\hline & 325 & -0.0 & 0.0761 & 0.0830 & 0.0802 & & 501 & -0.006687 & 0.0310 & 0.1220 & 0.1233 \\
\hline & 0.2752904 & & - & 0.0908 & & & & & & 0.2201 & - \\
\hline & & & - & & 0.030 & & & -0 . & & 0.2328 & \\
\hline & & & & & - & & & -0. & & 0.1075 & .0572 \\
\hline & & -0 & 0.0378 & & & & & & & & \\
\hline & & & 0.0370 & 00 & & & & 0.00 & 0.1144 & 0.1728 & 0.0827 \\
\hline & & 005552 & 0.0287 & & & & & -0.000506 & 0.0234 & 0.2063 & 0.03 \\
\hline & & & - & & & 9.5606 .348 & 0.2909644 & 0.071233 & 0.0226 & 0.1168 & 0.0209 \\
\hline 5.5497 .3874 & 0.2680527 & 8829 & 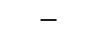 & 0.1566 & (2) & & & & & & \\
\hline
\end{tabular}

Note: the amplitude of the main pulsation component is denoted by $A_{0}$. The modulation amplitudes are $A_{+}$and $A_{-}$, corresponding to the larger

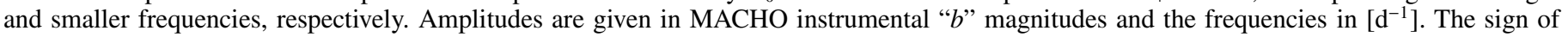
the modulation frequency $f_{\mathrm{BL}}$ is positive, if $A_{-}<A_{+}$, and negative, if $A_{-}>A_{+}$. For BL2 stars, $f_{\mathrm{BL}}$ stands for the average of the two modulation frequencies. This table also appears at the CDS (http://cdsweb.u-strasbg.fr). 